\title{
Selective PROTAC-mediated degradation of SMARCA2 is efficacious in SMARCA4 mutant cancers
}

Robert Yauch ( $\sim$ bobyauch@gene.com )

Genentech, Inc. https://orcid.org/0000-0001-9478-6283

Jennifer Cantley

Arvinas, LLC

Xiaofen Ye

Genentech

Emma Rousseau

Arvinas, LLC

Tom Januario

Genentech

Brian Hamman

HotSpot Therapeutics

Chris Rose

Genentech https://orcid.org/0000-0002-7502-3368

Tommy Cheung

Genentech

Trent Hickle

Genentech

Leofal Soto

Arvinas, LLC

Connor Quinn

Arvinas, LLC

Alicia Harbin

Arvinas, LLC

Elizabeth Bortolon

Arvinas, LLC

\section{Xin Chen}

Arvinas

\section{Roy Haskell}

Arvinas, LLC

Eva Lin 
Roche (United States)

\section{Shang-Fan Yu}

Geoff Del-Rosario

Genentech

\section{Emily Chen}

GENENTECH

\section{Scott Martin}

Roche (United States)

\section{Mark Merchant}

GENENTECH

\section{Matthew Grimmer}

Genentech

\section{Fabio Broccatelli}

Genentech

\section{Jing Wang}

Arvinas, LLC

\section{Jennifer Pizzano}

Arvinas, LLC

Peter Dragovich

Genentech

\section{Michael Berlin}

Arvinas, LLC

\section{Article}

Keywords:

Posted Date: January 27th, 2022

DOl: https://doi.org/10.21203/rs.3.rs-1133243/v1

License: (c) (1) This work is licensed under a Creative Commons Attribution 4.0 International License. Read Full License

Additional Declarations: Yes there is potential Competing Interest. Employees of Genentech or Arvinas, LLC

Version of Record: A version of this preprint was published at Nature Communications on November 10th, 2022. See the published version at https://doi.org/10.1038/s41467-022-34562-5. 


\section{Selective PROTAC-mediated degradation of SMARCA2 is efficacious in SMARCA4 mutant cancers}

Jennifer Cantley ${ }^{1}$, Xiaofen $\mathrm{Ye}^{2}$, Emma Rousseau ${ }^{1}$, Tom Januario ${ }^{2}$, Brian D. Hamman ${ }^{3}$, Chris M. Rose ${ }^{2}$, Tommy K. Cheung $^{2}$, Trent Hinkle ${ }^{2}$, Leofal Soto ${ }^{1}$, Connor Quinn ${ }^{1}$, Alicia Harbin ${ }^{1}$, Elizabeth Bortolon ${ }^{1}$, Xin Chen ${ }^{1}$, Roy Haskell $^{1}$, Eva Lin ${ }^{2}$, Shang-Fan Yu², Geoff Del Rosario ${ }^{2}$, Emily Chan ${ }^{2}$, Scott Martin², Mark Merchant ${ }^{2}$, Matt Grimmer $^{2}$, Fabio Broccatelli ${ }^{2}$, Jing Wang ${ }^{1}$, Jennifer Pizzano ${ }^{1}$, Peter S. Dragovich ${ }^{2}$, Michael Berlin ${ }^{1}$, Robert L. Yauch $^{2 *}$

\footnotetext{
${ }^{1}$ Arvinas, LLC, 5 Science Park, New Haven, CT 06511

${ }^{2}$ Genentech, 1 DNA Way, South San Francisco, 94080

${ }^{3}$ current address: HotSpot Therapeutics, Inc. 1 Deerpark Dr., Ste C, Monmouth Junction, NJ 08852

*email: bobyauch@gene.com
} 


\begin{abstract}
The mammalian SWI/SNF helicase SMARCA4 is frequently mutated in cancer and inactivation results in a cellular dependence on its paralog, SMARCA2, thus making SMARCA2 an attractive synthetic lethal target. However, published data indicates that achieving a high degree of selective SMARCA2 inhibition is likely essential to afford an acceptable therapeutic index, and realizing this objective has been challenging due to the homology with the SMARCA4 paralog. Herein we report the discovery of the first potent and selective SMARCA2 proteolysistargeting chimera (PROTAC) molecule (A947). Selective SMARCA2 degradation was achieved in the absence of selective SMARCA2/4 PROTAC binding and translated to potent in vitro growth inhibition and in vivo efficacy in SMARCA4 mutant models, compared to wild type models. Global ubiquitin mapping and proteome profiling revealed no unexpected off-target degradation related to A947 treatment. Our study thus highlights the ability to transform a non-selective SMARCA2/4-binding ligand into a selective and efficacious in vivo SMARCA2-targeting PROTAC, and thereby provides a potential new therapeutic opportunity for patients whose tumors contain SMARCA4 mutations.
\end{abstract}




\section{Introduction}

The multi-subunit switch/sucrose non-fermentable (SWI/SNF or BAF) complex facilitates the remodeling of chromatin to regulate key cellular processes including transcriptional regulation and DNA repair ${ }^{1,2}$. Catalytic function is conferred by one of two mutually exclusive ATP-dependent helicases, SMARCA2 and SMARCA4, which share strong protein sequence homology ${ }^{3}$. In addition to a highly conserved ATPase domain ( $93 \%$ identity), both proteins contain a conserved bromodomain (96\% identity) (BD) that can interact with acetylated chromatin. $\mathrm{SWI} / \mathrm{SNF}$ has gained a great deal of attention in cancer biology, as tumor sequencing studies have revealed that $\sim 20 \%$ of human cancers harbor mutations in specific core or accessory components of the complex ${ }^{4,5}$. In particular, loss of function mutations in SMARCA4 are enriched in subsets of multiple malignancies, with the highest prevalence of homozygous mutations occurring in non-small cell lung cancer (NSCLC) $)^{6,7}$. Aberrant chromatin remodeling caused by SMARCA4 mutations can result in the disruption of enhancer accessibility and accumulation of Polycomb repressive complexes 1 and 2 across the genome ${ }^{8,9}$.

Strategies to therapeutically target SMARCA4 mutant (SMARCA4 ${ }^{\text {mut }}$ ) cancers have focused on the identification of vulnerabilities that may be conferred in the context of the mutant state ${ }^{10}$. Most notably, functional genomic screens to identify gene dependencies have identified the paralog, SMARCA2, as a synthetic lethality in cancers with inactivated SMARCA4 ${ }^{11,12}$. Although the ATPase activity of SMARCA2 is required for the proliferation of SMARCA $4^{\mathrm{mut}}$ cells, bromodomain function is dispensible, as highlighted by the failure of inhibitors to the SMARCA2/4 bromodomain to suppress cell growth ${ }^{13}$. Such findings have subsequently led to efforts to discover ATPase inhibitors, however the currently described inhibitors are dual inhibitors of SMARCA2/4 and are hampered by dose-limiting tolerability issues, preventing the full in vivo exploration of anti-tumor activity ${ }^{14}$. Preclinical genetic studies would indicate that achieving selective inhibition of SMARCA2 will likely be essential for a successful therapeutic. Whereas the germline knockout of Smarca2 produced viable mice that are slightly larger than control littermates but show no other overt phenotypes, the knockout of Smarca4 is embryonic lethal and conditional deletion of Smarca4 has been associated with multiple tissue-specific phenotypes ${ }^{15-19}$. More importantly, the co-deletion of Smarca2 and Smarca4 in adult mice was lethal due to vascular defects ${ }^{20}$. Hence, SMARCA2 inhibitors with improved selectively over SMARCA4 will likely be required to achieve safe and maximal inhibition of SMARCA2 in this context.

Proteolysis targeting chimeras (PROTACs) represent an emerging therapeutic modality to induce the degradation of target proteins by recruiting the protein of interest to an E3 ubiquitin ligase, leading to the subsequent tagging of the protein for proteasome-mediated destruction through the addition of ubiquitin ${ }^{21,22}$. PROTACs offer several advantages over classical small molecule inhibitors, as they circumvent the requirement to employ ligands targeting the enzymatic function of the given target protein and they can function in a sub-stoichiometric manner enabling sustained pharmacodynamic effects at lower systemic exposures. Importantly, selective degradation using warhead ligands with nonselective binding properties has been demonstrated with PROTACs ${ }^{23,24}$. Although the mechanisms underlying selective degradation remain to be fully elucidated, the ability to form protein-protein interactions between the target protein and E3 ligase within the ternary complex can contribute to a more productive and selective degrader ${ }^{23,25}$. Hence, warhead selection and the choice of E3 ligase will play a critical role in determining whether selective degradation could be achieved using a PROTAC with equivalent binding affinities to multiple substrates.

In this study, we have used otherwise inert ligands with equivalent binding affinities to the bromodomains of SMARCA2/4 and the $5^{\text {th }}$ bromodomain of PBRM1 to develop a VHL-based PROTAC exhibiting potent and moderately selective degradation of SMARCA2. The VHL-SMARCA2 PROTAC can elicit enhanced growth inhibitory effects both in vitro and in vivo in SMARCA4 ${ }^{\text {mut }}$ cancer models relative to SMARCA4 wild-type (SMARCA4 ${ }^{\mathrm{wt}}$ ) models, in the absence of considerable tolerability issues. In contrast to previously described SMARCA2/4 ATPase inhibitor ${ }^{14}$ and PROTAC ${ }^{26}$, these findings provide the first example of a selective SMARCA2 targeting agent and provide pharmacologic support of this previously defined synthetic lethality in SMARCA4mut cancers. 


\section{Results}

To identify potent and selective PROTACs targeting SMARCA2, we linked a small-molecule ligand capable of binding the bromodomains of SMARCA2/4 and PBRM1 (5 $5^{\text {th }} \mathrm{BD}$ ) to a VHL-targeting moiety (Methods and M.B., P.D, manuscript in preparation). This work led to the identification of PROTAC, A947 (Fig. 1a). No difference in binding affinity to the SMARCA2 and SMARCA4 bromodomains was observed for A947 (Fig 1b). (SMARCA2 $\mathrm{K}_{\mathrm{d}}=93 \mathrm{nM}$, SMARCA4 $\mathrm{K}_{\mathrm{d}}=65 \mathrm{nM}$ ) A947 potently degraded SMARCA2 in SW1573 cells with a DC 50 (the drug concentration that results in 50\% protein degradation) of $39 \mathrm{pM}$ and achieving a maximal degradation of $96 \%$ at 10nM (Fig. 1c, Extended Data Fig. 1). In contrast, approximately 28 -fold higher concentration of A947 was needed to achieve a DC $_{50}$ on SMARCA4 $(1.1 \mathrm{nM})$, with a maximal degradation of SMARCA4 $(92 \%)$ being achieved at concentrations approaching $100 \mathrm{nM}$. This degree of degradative selectivity was maintained independent of the specific isoform of SMARCA2/4 evaluated (Fig. 1d, Extended Data Fig. 2). Furthermore, A947 exhibited similar selectivity on SMARCA2 degradation over PBRM1 (Extended Data Fig. 1). The cellular degradation of SMARCA2 by A947 required both SMARCA2 and VHL binding, as loss of SMARCA2 could be mitigated by the addition of excess free SMARCA2/4 or VHL -binding ligands (Fig 1e). In addition, a hydroxy-proline diastereomer of A947 expected to attenuate VHL binding (A857), as well as an analog lacking a critical phenol group in the SMARCA2-binding fragment (A858), were largely defective in degrading SMARCA2 in cells; with a negligible impact of A857 on SMARCA2 at the highest concentrations tested (Extended Data Fig. 3). The dependence on ubiquitination and proteosome-mediated degradation was demonstrated by the ability of an inhibitor to the ubiquitin activating enzyme (MLN-7243) and a proteasome inhibitor (MG-132) to block A947-mediated degradation of SMARCA2 (Fig. 1e). A947-mediated cellular degradation of SMARCA2 was rapid, with $~ 93 \%$ loss of the nuclear insoluble pool of SMARCA2 observed within 30 minutes (Extended Data Fig. 4). Finally, A947 was equally efficient in degrading both the murine and rat orthologs of SMARCA2, as assessed by monitoring the cellular degradation of these orthologs ectopically expressed in cells expressing endogenous human or murine VHL (Fig. 1f, Extended Data Fig. 5).

To more broadly assess the impact of A947 on the ubiquitylome in cells, we carried out quantitative di-glycine reminant profiling by mass spectrometry following treatment of SW1573 cells with a high $(500 \mathrm{nM})$ concentration of A957 to ensure maximal degradation of both SMARCA2/4 (Fig 2a, Supplementary Table 1). We observed ubiquitination of multiple lysines on both SMARCA2 and SMARCA4, with the strongest ubiquitination on K1450 mapping to the bromodomain of SMARCA2/4. Based on the recently elucidated cryo-EM structure of the BAF complex $^{27}$, the majority of the ubiquitination occurred on lysines mapping to the ATPase module and HSA domain, with no ubiquitination observed on very N-terminal lysines that are predicted to be anchored within the core complex. Importantly, we observed no ubiquitination of core BAF complex or accessory proteins. Globally, ubiquitination mediated at this high concentration of A947 in cells was specific to SMARCA2/4. In further support of the selectivity, we quantified degradation at the proteome level by mass spectrometry (Fig 2b, Supplementary Table 2). SMARCA2/4 and PBRM1 represented the only proteins impacted by A947. Taken together, the data indicate that A947 was highly specific for degrading the expected target proteins at high concentrations.

We next evaluated the impact of A947 on cell proliferation. In SMARCA4 ${ }^{\text {mut }}$ NCI-H1944 cells, A947 treatment resulted in the dose-dependent inhibition of growth that was dependent upon SMARCA2 degradation, as the VHL and SMARCA2/4 -binding defective analogs (A857 and A858, respectively) were significantly weaker in cells (Fig. 3a). To more broadly assess cellular activity and determine whether the moderate selectivity in degradation translated to selective effects on cell growth, we profiled a panel of lung cancer models characterized by SMARCA4 mutation status (Fig. 3b, Supplementary Table 3). Two additional cell lines that were deficient in SMARCA2/4 expression were included as further controls for any non-specific effect of A947. SMARCA4 ${ }^{\text {mut }}$ lung cancer cell lines were most sensitive to A947 treatment, with a median $\mathrm{IC}_{50}$ of $7 \mathrm{nM}$ across the panel of cell lines. We did not observe any relationship with the type of SMARCA4 variant and/or the position of the variant with cellular activity of A947 (Extended Data Fig. 6a). In contrast, SMARCA4 ${ }^{\text {WT }}$ cells were significantly less sensitive to A947, with a median IC 50 of $86 \mathrm{nM}$ across the panel of models evaluated. A947 treatment had no impact on growth of cells deficient in SMARCA2/4 expression. This differential in cellular sensitivity to A947 between SMARCA4 mutant and WT cells was also recapitulated upon longer treatment periods in clonogenic growth assays (Extended Data Fig. 6b). A947-mediated degradation resulted primarily in G1 arrest across SMARCA4 ${ }^{\mathrm{mut}}$ models that was not observed in control cell lines (Fig 3c, Extended Data Fig. 6c). We also did not observe evidence for acute cytotoxicity. At the transcriptional level, A947-mediated SMARCA2 degradation resulted primarily in transcriptional suppression in 
SMARCA4 ${ }^{\text {mut }}$ cells, consistent with the role of SMARCA2 as a chromatin regulator (Fig. 3d, Supplementary Table 4) (ref). Importantly, we observed a strong correlation $(r=0.53, p<2.2 \mathrm{e} 16)$ between the transcriptional changes occurring between A947 treatment compared to inducible SMARCA2 knockdown by shRNA, further supporting the on-target effect of A947 in cells.

To address whether A947 is active in vivo, we initially evaluated the pharmacodynamic (PD) effect following a single $40 \mathrm{mg}$ per kg intravenous (IV) dose of A947 in SMARCA4 ${ }^{\mathrm{mut}} \mathrm{HCC} 515$ xenografts over a 2 week period (Fig 4a, Extended Data Fig. 7). In addition to monitoring SMARCA2 protein levels, we evaluated 2 transcriptional target genes that were broadly regulated by SMARCA2 loss across SMARCA4 ${ }^{\text {mut }}$ models, KRT80 and PLAU. Both transcripts are directly regulated by SMARCA2, as previously defined by ChIP-seq and ATAC-seq studies ${ }^{6}$. The IV administration of A947 resulted in rapid reduction ( $96 \%$ reduction by 4 hours) in tumor SMARCA2 protein levels and achieved a maximal reduction by 24 hours. Decreases in the transcriptional readouts followed, achieving maximal target gene suppression by 96 hours post-dose. A slight rebound in SMARCA2 protein levels was observed over the 14 day period as tumor concentrations of A947 decreased; however due to prolonged A947 tumor exposures, these concentrations never reached baseline levels. Differential re-expression of KRT80 and PLAU transcripts was observed following one week after dose administration with PLAU mRNA levels paralleling SMARCA2 protein levels.

We subsequently took the dosing regimen of $40 \mathrm{mg}$ per kg every other week i.v. administration of A947 forward into efficacy studies in two different SMARCA4 ${ }^{\text {mut }}$ lung cancer xenograft models, HCC515 and HCC2302 (Fig. 4 b,c). Statistically-significant decreases in tumor growth were observed in both models, highlighted by near complete growth inhibition in the HCC515 model and 60\% tumor growth inhibition in the HCC2302 model. Tumor growth inhibition was dependent upon SMARCA2 degradation and was not due to non-specific effects of the chemical scaffold, as VHL and SMARCA2/4 -binding defective analogs A857 and A858 were not efficacious (Extended Data Fig. 8). Furthermore, tumor growth inhibition was observed in the absence of any appreciable loss in body weight at this dose and regimen, indicating that efficacy was not a consequence of in vivo toxicity (Extended Data Fig. 9a,b). Tumor pharmacodynamic biomarker responses were also measured at end of study; 24 hours after administration of a final dose. A947 treatment led to more than a 95\% decrease in tumor SMARCA2 protein levels in both models, however a slightly stronger suppression of KRT80 transcript was observed in the HCC515 model. To more extensively address the transcriptional impact of A947, we carried out RNAseq on mRNA isolated from these xenografts and monitored transcripts $(\mathrm{n}=412)$ that were determined from in vitro studies to represent acutelysuppressed and sustained targets of SMARCA2 loss common to both models (Fig. 4e, Supplementary Table 4). Consistent with the differential degree of KRT80 suppression, we observe slightly stronger suppression of SMARCA2-regulated genes in the HCC515 xenograft model, suggesting that slight differences in the pharmacodynamic effect may underlie the differences in efficacy between these models.

In order to address whether the tumor growth inhibition observed in SMARCA4 ${ }^{\text {mut }}$ models was due to a tumor cell autonomous effect of SMARCA2 degradation, we evaluated A947 administration in the SMARCA4 ${ }^{\text {wt }}$ Calu-6 xenograft model (Fig. 4f). Applying the same dose and regimen as used in the SMARCA4 ${ }^{\text {mut }}$ xenograft studies, A947 did not result in tumor growth inhibition in SMARCA $4{ }^{\text {wt }}$ Calu-6 xenografts despite achieving greater than 95\% degradation of SMARCA2 protein (Fig. 4g). Moderate degradation of SMARCA4 and PBRM1 was observed at the 24 hour post-last dose timepoint, with $58 \%$ and $57 \%$ decreases respectively. Although degradation selectivity can not be addressed in SMARCA4 ${ }^{\text {mut }}$ models due to the deficiency in human SMARCA4, we were able to monitor murine SMARCA4 protein levels within the tumor microenvironment of HCC515 and HCC2302 xenografts. Analogous to the Calu-6 xenografts, 57\% and 69\% reductions in murine SMARCA4 levels were observed in these studies, suggesting that murine SMARCA4 is degraded similarly to human SMARCA4 (Extended Data Fig. 10). Hence, the moderate selectivity for SMARCA2 over SMARCA4 degradation observed in vitro for A947 translated to a moderate degradative selectivity in vivo at this dose and regimen. Taken together, the data are supportive of a tumor cell intrinsic effect of SMARCA2 degradation and provide pharmacologic support of the synthetic lethal interaction.

Although SMARCA2 degraders would have the potential to be developed as single agents in the clinic to treat SMARCA $4^{\text {mut }}$ cancers, we have begun to address whether rational and ubiquitously active pharmacologic combinations exist for SMARCA2 degraders in SMARCA4 ${ }^{\text {mut }}$ cancers. To assess combination effects, we screened a library of 723 experimental and clinically approved agents in combination with A947 across 4 SMARCA4 ${ }^{\text {mut }}$ lung cancer cell lines (Fig 5a, Supplemental Table 5). Although A947 treatment sensitized to unique compounds in a 
given model, MCL1 inhibition represented the only combination exhibiting strong sensitization with A947 in more than one SMARCA4 ${ }^{\text {mut }}$ model ( 3 of the 4 models), as well as with multiple inhibitors. To confirm and extend this observation, we carried out a matrix titration of 2 separate MCL1 inhibitors (AMG-176 and S63845) with A947 and evaluated synergistic growth inhibition based upon the Bliss independence model in 5 SMARCA4 ${ }^{\text {mut }}$ models (Fig. 5b, Extended Data Fig. 11). In all cases, A947-mediated SMARCA2 degradation exhibited a synergistic interaction with MCL1 inhibition. Given the cytostatic effect of A947 treatment as a single agent and the anti-apoptotic function of MCL1, we evaluated whether the synergy was due to the ability of the combination to induce apoptosis. Indeed, live cell imaging of activated caspase 3/7 in SMARCA4 ${ }^{\mathrm{mut}}$ cells revealed that the combination was able drive these cells toward apoptotic cell death. These data, combined with previously published work leveraging a genetically-engineered degron tagging approach ${ }^{28}$, support a potential combination of SMARCA2 degraders with MCL1 inhibitors. 


\section{Discussion}

In this study, we report the first example of an exquisitely potent and moderately selective SMARCA2-targeting PROTAC, A947, that is active both in vitro and in vivo in SMARCA4 ${ }^{\text {mut }}$ NSCLC models. A947 exhibited a 28 -fold selectivity in degradation of SMARCA2 over SMARCA4 and notably exhibited no unexpected off-target effects at high concentration in both global ubiquitinome and proteome studies in cells. The ability of A947 to phenocopy the effect of inducible shRNA-mediated knockdown of SMARCA2 on transcriptome expression further supported the molecule's on target effect. A947 differs from a previously reported SMARCA2/4-targeting PROTAC, ACBI ${ }^{26}$. Despite differences in the models tested, A947 exhibited greater SMARCA2 degradation potency (39pM DC $50 \mathrm{~V}$. $6 \mathrm{nM} \mathrm{DC}_{50}$ ) and selectivity over SMARCA4 (28.2-fold v. 1.8-fold shift in $\left.\mathrm{DC}_{50}\right)^{26}$. This 28 -fold selectivity in cellular degradation achieved with A947 translated to selective tumor growth inhibition both in vitro and in vivo in SMARCA4 ${ }^{\text {mut }}$ cancers compared to SMARCA4 ${ }^{\text {wt }}$ cell line models. Pharmacodynamic profiling revealed that moderate in vivo degradation $(\sim 60 \%)$ of SMARCA4 could occur along with the associated strong SMARCA2 degradation $(>95 \%)$, however the former activity was not sufficient to either drive efficacy in a SMARCA $4{ }^{\mathrm{wt}}$ model nor to impact tolerability at the dosing regimen utilized. Taken together, these data provide the first pharmacologic support of this paralog-mediated, synthetic lethality; and provide an early indication that a potent SMARCA2 degrader with $\sim 30$-fold selectivity could be safely delivered.

An important aspect of this effort has been the ability to exploit non-selective SMARCA2/4 binding ligands to achieve selective cellular degradation in the context of the PROTAC. However, despite our ability to achieve selective cellular degradation, the mechanism underlying selectivity remains to be elucidated. Differences in ternary complex affinity have been attributed to several reported examples of selective degraders that utilize ligands with promiscuous binding properties ${ }^{23,24}$. Ternary complex formation has been previously observed with non-selective SMARCA2/4 PROTACs ${ }^{26}$ and we have similarly observed ternary complex formation using both the free bromodomains of SMARCA2 $2^{\mathrm{BD}}$ and SMARCA4 ${ }^{\mathrm{BD}}$, as well as the full-length SMARCA2 $2^{\mathrm{FL}}$ and SMARCA4 ${ }^{\mathrm{FL}}$ proteins, in the presence of VHL complex (VCB: VHL-ElonginC-ElonginB) and A947 (data not shown). However, we observed no significant difference in ternary complex affinity or complex half-life between VCB:A947:SMARCA2 and VCB:A947:SMARCA4 using either protein. It remains a possibility that isolated bromodomains or full-length proteins may not simulate the physiologically relevant substrate:PROTAC:VBC interface in the context of the native BAF complex. Selectivity could also be potentially driven by cell-intrinsic factors, as opposed to the biophysical properties of the ternary complex. We did consider whether differences in SMARCA2/4 resynthesis rate could contribute to the cellular degradation selectivity, however similar rates of resynthesis were observed in washout studies when compound was fully evicted from target binding in cells (data not shown). Nevertheless, understanding the biochemical and/or cellular mechanism of degradation selectivity of A947 remains an active area of research.

Due to their high molecular weight and poor physiochemical properties, the oral delivery of VHL-based PROTACs can be problematic. Preclinical efficacy studies have generally required intraperitoneal or subcutaneous administration of VHL-PROTACs at high doses and high frequencies, with few exceptions ${ }^{29}$. While the poor physiochemical properties of VHL-based PROTACs complicate their oral delivery, opportunities exist for alternative parenteral routes of administration ${ }^{30}$. Intravenous (IV) dosing offers some obvious advantages, such as the lack of physiological barriers to absorption, but requires solubility consistent with the target dose and an intravenous half-life ( $\left.\mathrm{T}_{1 / 2}\right)$ sufficiently long to satisfy the PKPD requirements and a likely intermittent dosing schedule. Prolonged IV T $1 / 2$ can be achieved by increasing PROTAC affinity to the lipid and phospholipid cell components (either via addition of a positive charge or increased lipophilicity), while decreasing or maintaining intrinsic metabolic stability ${ }^{31,32}$. The amine containing linker contained in A947 promotes high solubility in the IV formulation, as well as moderate $\mathrm{CL}(<16 \mathrm{ml} / \mathrm{min} / \mathrm{kg})$ and high affinity for body tissues in rodents, resulting in $\mathrm{VD}_{\mathrm{ss}}>6 \mathrm{~L} / \mathrm{kg}$ and $\mathrm{T}_{1 / 2}>6 \mathrm{hr}$ (data not shown). Interestingly, in rodents the residence time of A947 in tumors (and presumably in other body tissues) appears to be much higher than in plasma, suggesting that dissociation from tissues is a slower process compared to metabolic elimination from plasma (Fig. 4a, Extended Data Fig. 7). While these observations were not mechanistically investigated, they are consistent with intracellular lysosomal trapping, a phenomena frequently observed for lipophilic basic amines ${ }^{33}$. 
Overall, our data may have future clinical implications, offering a potential therapeutic option for patients harboring SMARCA4 ${ }^{\text {mut }}$ cancers. Although A947-mediated degradation of SMARCA2 results primarily in cytostasis that is consistent with prior genetic perturbation studies ${ }^{12}$, the overall depth of single-agent efficacy in the context of an immune-competent animal and/or upon prolonged dosing remains to be determined. Nevertheless, we considered whether rational drug combinations exist as a means to potentiate the tumor cell intrinsic, cellular activity of A947. We specifically sought to identify drug synergies that were broadly active and not necessarily cell context specific. Through pharmacologic profiling, we determined that inhibition of the BCL2-family pro-survival protein, MCL1, could synergize with SMARCA2 degradation across multiple SMARCA4 ${ }^{\text {mut }}$ models. Interestingly, MCL1 was additionally identified in a genome-wide CRISPR knockout screen to identify sensitizers to SMARCA2 loss in a genetically engineered system whereby SMARCA2 was endogenously tagged with the SMASh degron to enable degradation with the NS3 protease inhibitor, asunaprevir ${ }^{28}$. Drug sensitization was observed with additional antiapoptotic drugs including BCL-XL and IAP antagonists, however these effects were cell line specific. Combined with the prior report, these data may indicate the broader utility combining MCL1 antagonists with a SMARCA2 PROTAC. Multiple MCL1 inhibitors are under early clinical investigation and are being considered in combination with other therapeutics in solid tumors as a means to lower the apoptosis threshold ${ }^{34}$. 


\section{Figure Legends}

Fig. 1 A947 is a potent and moderately selective degrader of SMARCA2. a, Chemical structure of A947. b, Dose-response curves of A947 displacing a biotinylated SMARCA2/4-binding probe from recombinant SMARCA2 and SMARCA4 bromodomains. Data are presented as mean \pm s.d. from 4 replicates. c, Quantification of SMARCA2 and SMARCA4 protein levels by In Cell Western ${ }^{\mathrm{TM}}$ following 20h treatment of SW1573 cells. Data are normalized to DMSO control-treated cultures and presented as mean \pm s.d. from 7 independent experiments. d, Licor-based quantification of SMARCA2 and SMARCA4 isoforms ectopically expressed in TOV112D cells following 24h treatment with A947. Data is normalized to DMSO control treated cells. UniProt identifiers of the respective isoforms are shown in parentheses. e, Pretreatment (1h) of SW1573 cells with 20-fold molar excess of the respective inhibitors can block A947 $(500 \mathrm{nM})$-mediated degradation of SMARCA2. Total ubiquitin levels serve as a control for MLN-7243 and MG-132. HDAC1 serves as a loading control. f, Licor-based quantification of FLAGtagged orthologs (human, mouse, rat) of SMARCA2 ectopically expessed in human (TOV112D) and murine (LA4) cell lines upon $24 \mathrm{~h}$ treatment with a dose-response of A947. Data is normalized to levels of the respective SMARCA2 ortholog in control (DMSO) lysates.

Fig. 2 A947-mediated ubiquitination and degradation of SMARCA2/4. a, Global ubiquitylome changes, as assessed by di-glycine reminant mass spectrometry profiling, in SW1573 cells following treatment with 500nM A947 for 5 and 30 minutes. $n=5,720$ unique ubiquitinated peptides were identified and presented as $\log 2$ fold change relative to DMSO control cultures. Positions of ubiquitinated lysines in peptides that uniquely map to SMARCA2 (UniProtKB P-51531-1) or SMARCA4 (UniProtKB P-51532-2) are highlighted in blue and red, respectively. Peptides shared between SMARCA2/4 are shown in green, with ubiquitinated lysines mapped to the SMARCA2 sequence. b, Global proteome assessed by mass spectrometry following $8 \mathrm{~h}$ treatment with A947 $(100 \mathrm{nM})$ in SW1573 cells. Data is presented as a $\log 2$ fold change in the abundance of the respective proteins in two biological replicates. $\sim 8,900$ and $\sim 8,400$ proteins were quantified in replicate 1 and 2 , respectively.

Fig. 3. A947-mediated inhibition of cell proliferation in SMARCA4-mutant NSCLC cell lines. a, Viability of NCI-H1944 following 7 days of treatment with a dose response of A947 or binding-defective epimers. b, Effect of A947 treatment on the growth of 30 lung cancer cell lines defined by SMARCA4 or SMARCA2 status, represented as the concentration of A947 required to inhibit growth by $50 \%\left(\mathrm{IC}_{50}\right)$ following 7 days of treatment. Median $\mathrm{IC}_{50}$ 's are indicated by the black line. c, Cell cycle distribution following 48 hour treatment of a dose response of A947 across 4 SMARCA4-mutant lung cancer cell lines. d, $\log _{2}$-transformed fold change in mRNA expression values, as measured by RNA-seq, in HCC2302 cells treated for 96h with A946 compared to control DMSO treated cultures (xaxis), as well as HCC2302-shSMARCA2 cells treated with doxycycline for 168h compared to HCC2302 cells expressing a non-targeted control shRNA (shNTC). RNAseq data is representative of triplicated cultures. The correlation was calculated by the Pearson coefficient.

Fig. 4. A947 is selectively efficacious in SMARCA4-mutant NSCLC xenograft models. a, Tumor concentration (right y-axis) and pharmacodynamic biomarker responses (left y-axis) monitored over a two week period in HCC515 xenograft tumors following a single-dose, intravenous (i.v.) administration of A947 $(40 \mathrm{mg} / \mathrm{kg})$. Tumor levels of SMARCA2 protein were quantified by ImageLab and normalized to a loading control protein ( $\beta$-actin). Tumor levels of the mRNA transcripts, KRT80 and PLAU, were quantified by Taqman. Data is presented relative to levels in untreated tumors and represented as mean \pm s.d from $n=5$ animals per timepoint. $\mathbf{b}, \mathbf{c}$, Tumor volume in mice harboring either SMARCA4-mutant HCC515 (b) or HCC2302 (c) xenografts following administration of A947 (40mg/kg, i.v.) or vehicle control. Data is presented as mean \pm s.e.m. ( $\mathrm{n}=10 \mathrm{mice} / \mathrm{group})$. Statistical significance was assessed by a two-sided, unpaired Student t-test. d, Pharmacodynamic biomarker levels tumors collected at end of study from animals treated in b and c. Mice received a final dose of A947 24 hours prior to tumor collection. SMARCA2 protein and KRT80 mRNA levels were quantified as in a and presented relative to levels in the respective vehicle-treated tumors. e, Log-normalized counts per million reads (CPM) of SMARCA2 target genes determined by RNAseq and presented relative to levels in vehicle-treated tumors. A consensus set of SMARCA2-regulated genes $(\mathrm{n}=412)$ were defined based upon exhibiting acute $(24 \mathrm{~h})$ and sustained $(96 \mathrm{~h})$ suppression following A947 treatment of both models in vitro, as further described in the methods section. f, Tumor volume in mice harboring SMARCA4 wild-type Calu-6 xenografts following administration of A947 (40mg/kg, i.v.) or vehicle control. Data is presented as mean \pm s.e.m. ( $=10$ mice / group). Potential significance was assessed by a two-sided, unpaired Student t-test. n.s. nonsignificant. g, Levels of SMARCA2, SMARCA4 and PBRM1 
protein in Calu- 6 tumors were quantified by ImageLab and normalized to a loading protein control ( $\beta$-actin). Calu6 tumors were collected at the end of study from mice treated in $\mathbf{f}$ and mice received a final dose of A947 24 hours prior to tumor collection.

Fig. 5. A947-mediated SMARCA2 degradation synergizes with MCL1 inhibition. a, Cell viability in 4 SMARCA4 mutant NSCLC cell lines measured across a small-molecule library of 723 compounds screened as a dose-response in the presence or absence of $100 \mathrm{nM}$ A947 for 5 days. Data is plotted as the difference in the concentration required to inhibit growth by $50 \%\left(\Delta \mathrm{IC}_{50}\right)$ upon $-/+\mathrm{A} 947$ treatment. Two separate MCL1 inhibitors are annotated in red. b, Treatment of a representative SMARCA4-mutant cell line (NCI-H1793) with a 9x9 matrix titration of A947 with the MCL1 inhibitors, AMG-176 (upper plot) and S63845 (lower plot). Heatmaps depict activity in excess of the Bliss independence model to describe synergistic drug interactions. c, Live cell monitoring of apoptosis in SMARCA4-mutant NCI-H1944 (left graph) and NCI-H838 (right graph) cells grown in the presence of Caspase-3/7 Green Dye upon treatment with 100nM A947 and/or 1 $\mu$ M AMG-176. Data is presented apoptotic object count (mean \pm s.d) in triplicate cultures. 


\section{Extended Data Figure Legends}

Extended Data Fig 1. Immunoblot analysis of respective proteins following $18 \mathrm{~h}$ treatment of SW1573 cells with a dose-response of A947. HDAC1 serves as a loading control.

Extended Data Fig. 2. a, UniProtKB annotation of SMARCA2 and SMARCA4 isoforms. b, Schematic of SMARCA2 and SMARCA4 isoforms generated for ectopic expression studies. SMARCA4 isoform 3 (P51532-3) was not generated due to the fact that the amino acid differences annotated for this isoform were captured in the other SMARCA4 isoforms. c, Immunblot analysis of SMARCA2 and SMARCA4 isoform expression in human TOV112D cells that are deficient in endogenous SMARCA4. HDAC1 serves as a loading control.

Extended Data Fig. 3. Immunblot analysis of SMARCA2 following 18h treatment of SW1573 cells with A947 and control molecules defective in binding to SMARCA2/A4 (A858) and VHL (A857). HDAC1 serves as a loading control. The chemical structures of the respective molecules are shown, with modification sites highlighted in red.

Extended Data Fig. 4. a, Timecourse of A947 treatment on SMARCA2 protein levels in SW1573 cells. b, SMARCA2 immublots were quantified by Licor and presented as a percent degradation relative to the untreated control lysates. c, Immunoblot analysis of cytoplasmic, nuclear soluble and nuclear insoluble fractions of SW1573 evaluating SMARCA2 and VHL protein levels following 30min treatment with A947 (100nM). Lamin served as a control for the nuclear insoluble fraction and tubulin for the cytoplasmic fraction. Cellular fractionation was carried out as described in the Methods.

Extended Data Fig. 5. a, Immunoblots demonstrating FLAG-tagged SMARCA2 ortholog expression in human TOV112D and murine LA4 cells. HDAC1 serves as a loading control. b, Licor-based quantification of SMARCA2 levels in human (TOV112D) and murine (LA4) cell lines expressed SMARCA2 orthologs upon 24h treatment with a dose-response of A947. Data is normalized to levels of the respective SMARCA2 ortholog in control (DMSO) lysates.

Extended Data Fig. 6. a, Effect of A947 treatment on the growth of 28 lung cancer cell lines classified by SMARCA4 gene mutation variant types and data is represented as the concentration of A947 required to inhibit growth by $50 \%\left(\mathrm{IC}_{50}\right)$ following 7 days of treatment. FS, frameshift. b, Effect of a dose-response of A947 on clonogenic growth of a panel of 10 lung cancer cell lines defined by SMARCA4 mutation status. c, Cell cycle distribution following 48 hour treatment of a dose response of A947 across 2 SMARCA4-wt lung cancer cell lines and one SMARCA2-deficient model. Aphidicolin $(\mathrm{Aph}, 1 \mu \mathrm{M})$ treatment served as a control to block entry into $\mathrm{S}$ phase.

Extended Data Fig. 7. Plasma concentration (right y-axis) and pharmacodynamic biomarker responses (left y-axis) monitored over a two week period in HCC515 xenografts following a single-dose, intravenous (i.v.) administration of A947 $(40 \mathrm{mg} / \mathrm{kg})$. Tumor levels of SMARCA2 protein were quantified by ImageLab and normalized to a loading control protein ( $\beta$-actin). Tumor levels of the mRNA transcripts, KRT80 and PLAU, were quantified by Taqman. Data is presented relative to levels in untreated tumors and represented as mean \pm s.d from $n=5$ animals per timepoint.

Extended Data Fig. 8. Tumor volume in mice harboring either SMARCA4-mutant HCC515 xenografts following administration of A947 or control analogs defective in VHL (A857) and SMARCA2 (A858) binding (40 mg $/ \mathrm{kg}$, i.v., all groups). Data is presented as mean \pm s.e.m. ( $\mathrm{n}=8$ mice / group). Statistical significance was assessed by a twosided, unpaired Student t-test.

Extended Data Fig. 9. Body weight of mice harboring either SMARCA4-mutant HCC515 (a), HCC2302 (b) or Calu-6 (c) xenografts following administration $(40 \mathrm{mg} / \mathrm{kg}$, i.v.) of A947 or vehicle control. Data is presented as mean \pm s.e.m. ( $\mathrm{n}=10$ mice / group). Statistical significance of the A947 treated group was assessed by a two-sided, unpaired Student t-test.

Extended Data Fig. 10. a,b, Immunoblot analysis of HCC515 (a) and HCC2302 (b) tumors collected at end of study from animals treated in Fig. 4b and Fig. 4c, respectively. Mice received a final dose of A947 24 hours prior to 
tumor collection. The SMARCA4 signal represents the murine stromal infiltrate within these SMARCA4-mutant human xenografts. $\beta$-actin served as a loading control. The A947-mediated decrease in the protein signal relative to the vehicle control was determined through ImageLab-quantification of $\beta$-actin-normalized protein signal.

Extended Data Fig. 11. Treatment of 4 additional SMARCA4-mutant cell lines with a 9x9 matrix titration of A947 with the MCL1 inhibitors, AMG-176 (left plots) and S63845 (right plots). Heatmaps depict activity in excess of the Bliss independence model to describe synergistic drug interactions.

\section{Author Contributions}

J.C. and R.Y. designed, analyzed and interpreted experiments and wrote the manuscript, X.Y., E.R., T.J., T.C., T.H., L.S., C.Q., A.H., E.B., E.L., S.Y., G.R., E.C., M.G., designed, performed and analyzed experiments, C.R., S.M., M.M, F.B., J.W., J.P., P.D., M.B. designed, analyzed and interpreted experiments. All authors commented on the manuscript.

\section{Acknowledgements}

We would like to thank the following individuals for their assistance with reagent generation, experimentation and/or helpful discussions: Keith Anderson, Yvonne Kschonsak, Chad Liu, Mark Bookbinder, Greg Cadelina, Kim Davenport, Dean DiNicola, and Debbie Gordon. 
References

1. Valencia, A. M. \& Kadoch, C. Chromatin regulatory mechanisms and therapeutic opportunities in cancer. Nat Cell Biol 21, 152-161 (2019).

2. Kadoch, C. \& Crabtree, G. R. Mammalian SWI/SNF chromatin remodeling complexes and cancer: Mechanistic insights gained from human genomics. Science Advances 1, e1500447-e1500447 (2015).

3. Mashtalir, N. et al. Modular Organization and Assembly of SWI/SNF Family Chromatin Remodeling Complexes. Cell 175, 1272-1288.e20 (2018).

4. Shain, A. H. \& Pollack, J. R. The Spectrum of SWI/SNF Mutations, Ubiquitous in Human Cancers. Plos One 8, e55119 (2013).

5. Mittal, P. \& Roberts, C. W. M. The SWI/SNF complex in cancer - biology, biomarkers and therapy. Nat Rev Clin Oncol 17, 435-448 (2020).

6. Fernando, T. M. et al. Functional characterization of SMARCA4 variants identified by targeted exomesequencing of 131,668 cancer patients. Nat Commun 11, 5551 (2020).

7. Schoenfeld, A. J. et al. The Genomic Landscape of SMARCA4 Alterations and Associations with Outcomes in Patients with Lung Cancer. Clin Cancer Res 26, 5701-5708 (2020).

8. Stanton, B. Z. et al. Smarca4 ATPase mutations disrupt direct eviction of PRC1 from chromatin. Nat Genet 49, 282-288 (2017).

9. Hodges, H. C. et al. Dominant-negative SMARCA4 mutants alter the accessibility landscape of tissueunrestricted enhancers. Nat Struct Mol Biol 25, 61-72 (2018).

10. Wanior, M., Krämer, A., Knapp, S. \& Joerger, A. C. Exploiting vulnerabilities of SWI/SNF chromatin remodelling complexes for cancer therapy. Oncogene 40, 3637-3654 (2021).

11. Oike, T. et al. A Synthetic Lethality-Based Strategy to Treat Cancers Harboring a Genetic Deficiency in the Chromatin Remodeling Factor BRG1. Cancer Research 73, 5508-5518 (2013).

12. Hoffman, G. R. et al. Functional epigenetics approach identifies BRM/SMARCA2 as a critical synthetic lethal target in BRG1-deficient cancers. Proc. Natl. Acad. Sci. U.S.A. 111, 3128-3133 (2014).

13. Vangamudi, B. et al. The SMARCA2/4 ATPase Domain Surpasses the Bromodomain as a Drug Target in SWI/SNF-Mutant Cancers: Insights from cDNA Rescue and PFI-3 Inhibitor Studies. Cancer Research 75, 3865$3878(2015)$.

14. Papillon, J. P. N. et al. Discovery of Orally Active Inhibitors of Brahma Homolog (BRM)/SMARCA2 ATPase Activity for the Treatment of Brahma Related Gene 1 (BRG1)/SMARCA4-Mutant Cancers. J Med Chem 61, 10155-10172 (2018).

15. Reyes, J. C. et al. Altered control of cellular proliferation in the absence of mammalian brahma (SNF2 $\alpha$ ). Embo $J$ 17, 6979-6991 (1998). 
16. Bultman, S. et al. A Brg1 Null Mutation in the Mouse Reveals Functional Differences among Mammalian SWI/SNF Complexes. Mol Cell 6, 1287-1295 (2000).

17. Holik, A. Z. et al. Brg1 is required for stem cell maintenance in the murine intestinal epithelium in a tissuespecific manner. Stem Cells 31, 2457-2466 (2013).

18. Sokpor, G., Xie, Y., Rosenbusch, J. \& Tuoc, T. Chromatin Remodeling BAF (SWI/SNF) Complexes in Neural Development and Disorders. Front Mol Neurosci 10, 243 (2017).

19. Hang, C. T. et al. Chromatin regulation by Brg1 underlies heart muscle development and disease. Nature 466, 62-67 (2010).

20. Willis, M. S. et al. Functional Redundancy of SWI/SNF Catalytic Subunits in Maintaining Vascular Endothelial Cells in the Adult Heart. Circ Res 111, e111-e122 (2012).

21. Chamberlain, P. P. \& Hamann, L. G. Development of targeted protein degradation therapeutics. Nat Chem Biol 15, 937-944 (2019).

22. Neklesa, T. K., Winkler, J. D. \& Crews, C. M. Targeted protein degradation by PROTACs. Pharmacol Therapeut 174, 138-144 (2017).

23. Bondeson, D. P. et al. Lessons in PROTAC Design from Selective Degradation with a Promiscuous Warhead. Cell Chem Biol 25, 78-87.e5 (2018).

24. Smith, B. E. et al. Differential PROTAC substrate specificity dictated by orientation of recruited E3 ligase. Nat Commun 10, 131 (2019).

25. Gadd, M. S. et al. Structural basis of PROTAC cooperative recognition for selective protein degradation. Nat Chem Biol 13, 514-521 (2017).

26. Farnaby, W. et al. BAF complex vulnerabilities in cancer demonstrated via structure-based PROTAC design. Nat Chem Biol 15, 672-680 (2019).

27. Mashtalir, N. et al. A Structural Model of the Endogenous Human BAF Complex Informs Disease Mechanisms. Cell 183, 802-817.e24 (2020).

28. Rago, F. et al. Degron mediated BRM/SMARCA2 depletion uncovers novel combination partners for treatment of BRG1/SMARCA4-mutant cancers. Biochem Bioph Res Co 508, 109-116 (2018).

29. Watt, G. F., Scott-Stevens, P. \& Gaohua, L. Targeted protein degradation in vivo with Proteolysis Targeting Chimeras: Current status and future considerations. Drug Discov Today Technologies 31, 69-80 (2019).

30. Doak, B. C., Over, B., Giordanetto, F. \& Kihlberg, J. Oral Druggable Space beyond the Rule of 5: Insights from Drugs and Clinical Candidates. Chem Biol 21, 1115-1142 (2014).

31. Rodgers, T. \& Rowland, M. Mechanistic Approaches to Volume of Distribution Predictions: Understanding the Processes. Pharmaceut Res 24, 918-933 (2007).

32. Broccatelli, F., Aliagas, I. \& Zheng, H. Why Decreasing Lipophilicity Alone Is Often Not a Reliable Strategy for Extending IV Half-life. Acs Med Chem Lett 9, 522-527 (2018). 
33. Schmitt, M. V., Lienau, P., Fricker, G. \& Reichel, A. Quantitation of lysosomal trapping of basic lipophilic compounds using in vitro assays and in silico predictions based on the determination of the full $\mathrm{pH}$ profile of the endo-/lysosomal system in rat hepatocytes. Drug Metab Dispos 47, dmd.118.084541 (2018).

34. Bolomsky, A. et al. MCL-1 inhibitors, fast-lane development of a new class of anti-cancer agents. $J$ Hematol Oncol 13, 173 (2020). 


\title{
Methods
}

\section{Chemical Compounds}

A947, A857, A858 and the free VHL ligand (A2702) were prepared by Arvinas, Inc., with detailed chemical synthesis described at the end of this Methods section. The chemical compounds MLN-7243 and MG-132 were obtained from SelleckChem. The SMARCA4/2 bromodomain inhibitor (example 47, WO2016138114, https://patents.google.com/patent/WO2016138114A1/en) was used in competition studies (Fig. 1e).

\begin{abstract}
Antibodies
The following antibodies were utilized: SMARCA2 (Cell Signaling, 11966), SMARCA4 (Abcam, ab110641), PBRM1 (Bethyl Labs, A301-591A), SMARCC1 (Cell Signaling, 11956), HDAC1 (Cell Signaling, 34589), VHL (Cell Signaling, 68547), FLAG (Sigma, F3165), Lamin A/C (Cell Signaling, 4777), $\alpha$-Tubulin (Sigma, T6074), $\beta$ Tubulin (Cell Signaling, 2128), $\beta$-Actin (Cell Signaling, 3700 and 4970) and total ubiquitin (Cell Signaling, 3936).
\end{abstract}

\section{Cell lines and cell culture}

Cell lines were obtained from the following sources (indicated in Supplemental Table 3): American Type Culture Collection (ATCC), Japanese Collection of Research Bioresources Cell Bank (JHSF), Deutsche Sammlung von Mikroorganismen und Zellkulturen (DSMZ), Riken, or licensed from UT Southwestern (UTSW). Cells were maintained in RPMI1640 supplemented with 10\% Fetal Bovine Serum (FBS) and 2mM L-Glutamine, under 5\% $\mathrm{CO} 2$ at $37^{\circ} \mathrm{C}$, with the exception of SW1573 (DMEM) and Calu-6 (EMEM). Cell line identity was verified by highthroughput single nucleotide polymorphism (SNP) genotyping using Illumina Golden Gate multiplexed assays. SNP profiles were compared to SNP calls from internal and external databases to determine or confirm ancestry.

\begin{abstract}
AlphaLISA $®$
AlphaLISA ${ }^{\circledR}$ studies were carried out using histidine (His)-tagged recombinant human SMARCA2 (aa.1377-1486; NP_620614) or SMARCA4 (aa. 1448-1575; NP_003063) proteins expressed in Escherichia coli. Compounds were diluted with 3-fold dilutions (11-point) in 96-well plates with a top concentration of 3mM in $100 \%$ DMSO.

Compounds were further diluted 10-fold in Alpha LISA buffer consisting of 50mM HEPES (Life Technologies 15630-080), 50mM NaCl (Sigma BCBW5699), 69uM Brij (Sigma SLBM8986V), and 0.1 mg/ml BSA (Sigma A7906-100G) brought up to $100 \mathrm{~mL}$ final volume in water (Sigma RNBG4333), resulting in a top concentration of $0.3 \mathrm{mM}$ in $10 \%$ DMSO. $3 \mathrm{uL}$ of this dilution was spotted into 384 well plate(s) (Perkin Elmer 6007290), final reaction volume of $30 \mathrm{uL}$, with compounds having a final top concentration of $30 \mathrm{uM}$. The final $30 \mathrm{ul}$ reaction consisted of the following components: Compound, 7nM His-SMARCA2 (or His-SMARCA4), 20nM SMARCA2/SMARCA4 Biotin Probe (example 248, WO2016138114), 1:400 Dilution of anti-His Alpha-LISA Acceptor Beads (Perkin Elmer AL128M), and 1:400 Dilution of Streptavidin Alpha-LISA Donor Beads (Perkin Elmer 6760002). To make a working stock of His-SMARCA2/SMARCA4 and biotinylated probe, $1.7 \mathrm{uL}$ of $60 \mathrm{uM}$ His-SMARCA2 (or His-SMARCA4) stock and 30uL of 10uM biotinylated probe (diluted in Alpha LISA buffer) was added to $20 \mathrm{~mL}$ Alpha LISA buffer to give final concentrations of $17.5 \mathrm{nM}$ protein and $50 \mathrm{nM}$ probe. Mixtures were then incubated at room temperature for 5-10 minutes. Then $12 \mathrm{uL}$ of protein/probe mixture was added to each reaction well in 384 well plate containing compounds (or probe only for background control wells). Plates were incubated for 10-15 minutes at room temperature. Anti-His6x Acceptor beads were diluted 100X in Alpha-LISA Buffer protected from light and $7.5 \mathrm{uL}$ added to each well. Plates were incubated 10-15 minutes at room temperature protected from light. Streptavidin donor beads were diluted 100X in Alpha-LISA Buffer protected from light and 7.5 $\mathrm{uL}$ added to each well. Plates were incubated for at least 15 minutes (no more than 4 hours) at room temperature protected from light and plates read at $615 \mathrm{~nm}$ on a micro-plate reader. For data analysis, the averages of control wells (probe + protein only max signal and probe only background control) were calculated and emission values at $615 \mathrm{~nm}$ were used to calculate percent displacement values using this formula:

(probe/protein - probe /protein /compound) / (probe/protein - probe only) $* 100=\%$ displacement Other values, such as mean and standard deviation, were calculated using GraphPad Prism software package.
\end{abstract}

\section{In-Cell Western}

SW1573 cells were seeded at 8000/well in 96-well black/clear-bottom plates with 180ul DMEM containing 1\% penstrep, $1 \%$ HEPES and $10 \%$ FBS and incubated overnight at $37^{\circ} \mathrm{C}$ to allow adherence. The next morning cells were 
treated with $20 \mathrm{uL}$ of $10 \mathrm{x}$ compound and incubated for an additional 20hrs. Cells were then washed with ice cold DPBS, then 50uL of ice cold 4\% PFA (Electron Microscopy Sciences 15711)/DPBS was added, and plates were then incubated at RT for 20 minutes. PFA was then removed and $200 \mathrm{uL}$ of TBS-T containing $0.5 \%$ Triton X 100 (Sigma T8787) was added. Plates were incubated at RT for 30 minutes. TBS-T/Triton X was then removed, 50uL of Li-cor blocking solution (Li-Cor 927-50003) was added, and plates were incubated at RT for 1hr. Blocking solution was removed, $50 \mathrm{uL}$ of Li-Cor blocking solution containing primary antibody to SMARCA2 (1:2000) and $\alpha$-Tubulin (1:2000) or SMARCA4 $(1: 1000)$ and $\alpha$-Tubulin $(1: 2000)$ was added, and plates were incubated at $4^{\circ} \mathrm{C}$ overnight. The next day, plates were washed $3 \mathrm{x}$ with TBS-T and 50uL of secondary antibody cocktail in Li-Cor blocking solution was added (IRDye 800CW Goat anti-rabbit IgG, Li-Cor 926-32211 and IRDye 680RD Goat anti-mouse IgG, Li-Cor 926-68070; both secondary antibodies are diluted 1:5000). Plates were incubated at RT for $1 \mathrm{hr}$ protected from light. Then plates were washed twice with TBS-T and excess liquid removed. Plates were read on the Li-Cor Odyssey with default intensity setting of 5.0 for both channels. Li-Cor images were analyzed with the in-cell Western feature of Image Studio Lite. Assays were run with technical duplicates and multiple biological replicates $(>2)$ with error calculated with GraphPad prism using 95\% confidence interval.

\section{Immunoblotting (in vitro samples)}

Following treatment of cells as indicated, cells were lysed in RIPA buffer containing $0.5 \mathrm{M} \mathrm{NaCl}$, then homogenized for 3 mins at speed 10 (NextAdavance, Bullet Blender $\left.{ }^{R} 24\right)$. Proteins (12ug or 18ug) were resolved on 4-12\% BisTris or 3-8\% Tris-Acetate gels and transferred to nitrocellulose membranes by iBlot. Membranes were incubated overnight with primary antibodies as indicated. IRDye ${ }^{\mathrm{R}}$-conjugated secondary antibodies were used to bind primary antibodies and images were visualized on the Odyssey Imager (LI-COR).

\section{SMARCA2/4 isoform and ortholog expression}

The pLenti6.3 vector system was used for all ectopic expression experiments. All DNA constructs were generated with a C-terminal FLAG tag. The human SMARCA2 and SMARCA4 isoform sequences are annotated in Extended Data Figure 2. For SMARCA2 orthologs, the following sequences were utilized: human (NM_139045.2), rat (XM_006231227.3), and mouse (NM_011416.2). The packaging and envelope vectors, $\Delta 8.91$ and VSV.G were cotransfected with pLenti6.3-based constructs into 293T cells by using lipofectamine 2000 (Invitrogen). Media containing lentiviral particles was collected 3 days post transfection, filtered through $0.45 \mu \mathrm{m}$ filters. Cells

(TOV112D (human) and/or LA-4 (murine)) were transduced with pLenti6.3- constructs particles, and selected with $8 \mu \mathrm{g} / \mathrm{mL}$ blasticidin (Gibco, A1113903) 3 days after transduction.

\section{Generation of inducible SMARCA2 shRNA cells}

Inducible SMARCA2 knockdown cell lines were generated using shRNAs directed against SMARCA2 tandemly delivered in a modified pBH1.2 piggy-bac system (Smarca2_iKD_dual62_pBH1.2). The following shRNAs were utlilized: shRNA6:GATCCGTCTCGTCGAGCAATCATTTGGTTGTAGTGAAATAtATATTAAACAACCAAATGATTG CTCGACGTTACGGTAC and shRNA2:GATCCGTCTGACTGTTCACGTTCATCCTGGTAGTGAAATAtATATTAAACCAGGATGAACGTG AACAGTCTTACGGTAC.

pBO (Piggybac transposase) were co- transfected with Smarca2_iKD_dual62_pBH1.2 into HCC2302 cells by using lipofectamine 2000 (ThermoFisher Scientific). Cells were selected with $2 \mu \mathrm{g} / \mathrm{mL}$ puromycin (Gibco) 3 days after transfection and subsequently subcloned.

\section{Sample preparation for quantitative proteome and ubiquitylome analysis}

Cells were lysed on plate using a lysis buffer consisting of $9 \mathrm{M}$ urea, $50 \mathrm{mM}$ HEPES (pH 8.5), and complete-mini (EDTA free) protease inhibitor (Roche). Protein concentrations were then estimated by BCA assay (ThermoFisher Pierce, Rockford, IL). Disulfide bonds were reduced with $5 \mathrm{mM}$ DTT $\left(45 \mathrm{~min}, 37^{\circ} \mathrm{C}\right.$ ), followed by alkylation of cysteine residues by $15 \mathrm{mM}$ IAA (30 min, RT Dark), and finally capped by the addition of $5 \mathrm{mM}$ DTT (15 min, RT Dark). Proteins were then precipitated by chloroform/methanol precipitation and resuspended in digestion buffer (8 $\mathrm{M}$ urea, $50 \mathrm{mM}$ HEPES $\mathrm{pH}$ 8.5). Samples were diluted to $4 \mathrm{M}$ urea before initial protein digestion was performed by the addition of 1:100 LysC followed by incubation at $37^{\circ} \mathrm{C}$ for 3 hours. Samples were then diluted to $1.5 \mathrm{M}$ urea 
with $50 \mathrm{mM}$ HEPES ( $\mathrm{pH} 8.5$ ) before the addition of 1:50 Trypsin and incubation overnight at room temperature. Peptide mixtures were acidified and desalted via solid phase extraction (SPE; SepPak - Waters, Boston, MA). For global proteome analysis, peptides were resuspended in $200 \mathrm{mM}$ HEPES (pH 8.5) and a $100 \mu \mathrm{g}$ aliquot of peptides was mixed with tandem mass tags (TMT or TMTpro, ThermoFisher Pierce, Rockford, IL) at a label to protein ratio of 2:1. After 1 hour of labeling, the reaction was quenched by the addition of $5 \%$ hydroxylamine and incubated at room temperature for $15 \mathrm{~min}$. Labeled peptides were then mixed, acidified, and purified by SPE. Samples were separated by offline high $\mathrm{pH}$ reversed-phase fractionation using an ammonium formate based buffer system delivered by an 1100 HPLC system (Agilent). Peptides were separated over a $2.1 \times 150 \mathrm{~mm}, 3.5 \mu \mathrm{m}$ 300Extend-C18 Zorbax column (Agilent) and separated over a 75 -minute gradient from $5 \% \mathrm{ACN}$ to $85 \% \mathrm{ACN}$ into 96 fractions. The fractions were concatenated into 24 samples of which 12 or 24 were analyzed for proteome quantification. Fractions were concatenated by mixing different parts of the gradient to produce samples that would be orthogonal to downstream low $\mathrm{pH}$ reversed phase LC-MS/MS. Combined fractions were dried, desalted by SPE, and dried again.

For ubiquitylome analysis, peptides were resuspended in $1 \mathrm{X}$ detergent containing IAP buffer (Cell Signaling Technology), cleared by high speed centrifugation (18,000 x g, 10 minutes) and enriched using an automated procedure previously described ${ }^{1}$. Enriched ubiquitinated peptides were prepared for multiplexed quantitative analysis as previously described except that TMTpro reagents were used ${ }^{2}$. All six fractions were analyzed by LC$\mathrm{MS} / \mathrm{MS}$.

\section{Quantitative mass spectrometry and data analysis}

Quantitative mass spectrometry analysis was performed on an Orbitrap Fusion Lumos or Orbitrap Eclipse mass spectrometer (ThermoFisher, San Jose, CA) coupled to a Waters NanoAcquity (Waters, Milford, MA) or Thermo Ultimate 3000 RSLCnano ProFlow (ThermoFisher, San Jose, CA) HPLC. Peptides were separated over a $100 \mu \mathrm{m} \mathrm{X}$ $250 \mathrm{~mm}$ PicoFrit column (New Objective) packed with $1.7 \mu \mathrm{m}$ BEH-130 C18 (Waters, Milford, MA) at a flow rate of 450 or $500 \mathrm{~nL} / \mathrm{min}$ or over a $25 \mathrm{~cm}$ IonOpticks Aurora column (IonOpticks, Fitzroy, Australia) at $300 \mathrm{~nL} / \mathrm{min}$ for a total run time of $180 \mathrm{~min}$. The gradient started at $2-5 \% \mathrm{~B}(98 \% \mathrm{ACN}, 1 \% \mathrm{FA})$ and ended at $30 \% \mathrm{~B}$ over 140 minutes and then to $50 \% \mathrm{~B}$ at $160 \mathrm{~min}$.

Peptides were surveyed via Orbitrap FTMS1 analysis $(120,000$ resolution, AGC $=1 \times 106$, maximum injection time $[\mathrm{max} \mathrm{IT}]=50 \mathrm{~ms}$ ) and the most intense 10 peaks were selected for MS/MS ensuring that any given peak was only selected every 35 or 45 seconds (tolerance $=10 \mathrm{ppm}$ ). For all runs, "one precursor per charge state" was ON. For data collected on the Eclipse mass spectrometer, Advanced Precursor Detection (APD), FAIMS (CVs = -50, -70), and the Precursor Fit Filter (70\% fit and 0.5 fit window) were employed.

For peptide identification, precursors were isolated using the quadrupole ( $0.5 \mathrm{Th}$ window), fragmented using CAD $(\mathrm{NCE}=35$ for TMT and $\mathrm{NCE}=30$ for TMTpro) and analyzed in the ion trap using a Turbo speed scan (AGC $=$ $\left.2 \times 10^{4}, \operatorname{maxIT}=100 \mathrm{~ms}\right)$ for proteome analysis or an Orbitrap scan at 15,000 resolution $\left(\mathrm{AGC}=7.5 \times 10^{4}\right.$, $\operatorname{maxIT}=$ $200 \mathrm{~ms}$ ) for ubiquitylome analysis. A real-time database search was utilized for both proteome and ubiquitylation quantification on the Eclipse mass spectrometer. The real-time database search performed an in silico trypsin digest with full specificity and 1 missed cleavage and used concatenated decoy proteins to calculate FDR in real time. The precursor PPM tolerance was set to $10 \mathrm{ppm}$ and the real-time search static and dynamic modifications matched the search parameters below. For quantitative SPS-MS3 analysis, the top 8 ions were simultaneously selected (synchronous precursor selection - SPS, $\mathrm{AGC}=1.5 \times 10^{5}$ or $3.0 \times 10^{5}$ [proteome] or $4.0 \times 10^{5}$ [ubiquitylome], $\max$ IT $=$ $150 \mathrm{~ms}$ [proteome] or $400 \mathrm{~ms}$ [ubiquitylome]) and fragmented by HCD (NCE=55 [TMT] or 40 [TMTpro]) before analysis in the Orbitrap (resolution $=50,000$ ).

All mass spectrometry data was searched using Mascot against a concatenated target-decoy human database (downloaded June 2016) containing common contaminant sequences. For the database search a precursor mass tolerance of $25 \mathrm{ppm}$ (TMTpro) or $50 \mathrm{ppm}$ (TMT), fragment ion tolerance of $0.5 \mathrm{Da}$ (TMTpro) or $0.8 \mathrm{Da}$ (TMT), and 1-2 (proteome) or 3 (ubiquitylome) missed cleavages. Carbamidomethyl cysteine (+57.0214) and TMT labeled nterminus ( +229.1629 for TMT and +304.2071 for TMTpro) were applied as static modifications for all analyses. For proteome analysis, TMT or TMTpro on lysine was also set as a static modification. Methionine oxidation $(+15.9949)$ and TMT or TMTpro on tyrosine were set as a dynamic modifications for all searches. For ubiquitylome searches, TMTpro on lysine and TMTpro-KGG on lysine (+418.2510) were considered as variable modifications. Peptide spectral matches for each run were filtered using line discriminant analysis (LDA) to a false discovery rate (FDR) of $2 \%$ and subsequently as an aggregate to a protein level FDR of $2 \%$.

Quantification and statistical testing of TMT proteomics data was performed using MSstats ${ }^{3}$. Prior to MSstats analysis, peptide spectral matches (PSMs) were filtered to remove matches from decoy proteins; peptides with length less than 7; with isolation specificity $<50 \%$; with reporter ion intensity $<256$; and with summed reporter ion 
intensity (across all channels) $<30,000$. Additionally, to separate peptides shared between SMARCA2 and SMARCA4, peptides matching to either protein were labeled as SMARCA2, SMARCA4, or SMARCA2/4 prior to MSstats analysis. This enables quantification of shared peptides to be performed as if they were a separate protein group. In the case of redundant PSMs (i.e. multiple PSMs in one MS run that map to the same peptide), PSMs were summarized by the maximum reporter ion intensity per peptide and channel and median equalized. In the case of redundant PSMs across fractions (i.e., redundant matching PSMs being found in multiple fractionated runs), PSMs were summarized by selecting the fraction with the maximum reporter ion intensity for each PSM. Protein level summarization was performed using a Tukey median polish approach. Differential abundance analyses between conditions were performed in MSstats based on a linear mixed-effects model per protein, and resulting $\mathrm{p}$-values adjusted for multiple hypothesis testing by using the Benjamini-Hochberg procedure. For ubiquitylome analysis, the $\log 2$ ratio values of each ubiquitinated peptide were normalized to the corresponding protein measurement before visualization; if a protein $\log 2$ ratio was not measure the ubiquitylation measurement was carried forward unchanged.

\section{Cell Viability Assays}

Cells were plated in 96-well plates at 500 cells per well and treated with a dose range of A947 starting with a highest concentration of 500nM. After incubating for 7 days, viability was measured using CellTiter-Glo (Promega) reagent. Reagent was added directly to the cells at a $1 / 1$ ratio of reagent to cell culture medium. Following a 15 minute incubation, luminescence was measured using the multimode plate reader EnVision 2105 (PerkinElmer). Viability was normalized to DMSO treated control cells. Cell viability experiments were performed in triplicate cultures.

\section{Clonogenic assay}

Cells (1500-5000, depending on doubling time) were plated in 12-well plates for 24 hours prior to treatment with fresh media containing compounds at indicated concentrations. Fresh media containing compound was replaced every 3 to 4 days until cells reached confluence to stop culture. Colonies were visualized by staining with $0.5 \%$ crystal violet for 20 minutes at room temperature.

\section{Cell cycle analysis}

Cell lines were treated for 48 hours with a dose response of A947 prior to pulsing for 15 minutes with 10uM EdU. Cells were subsequently fixed in $4 \%$ paraformaldehyde for 10 minutes, washed 3 times with PBS and then blocked and permeabilized in PBS containing 10\% FBS, $1 \%$ BSA, $0.1 \%$ TX-100, and $0.01 \%$ NaN3 for 1 hour at room temperature. Permeabilization buffer was removed and the cells were washed $3 \mathrm{x}$ with PBS. The Click-iT® reaction was perform according to the manufacturer's (Invitrogen C10337) protocol. Following a 30 minute incubation in the dark, the cells were washed 3 times with PBS. For nuclear staining, cells were treated with Hoechst 33342 (ThermoFisher) at 1:10000 for ten minutes at room temperature. Cells were then washed again 3 times with PBS and imaged on Opera Phenix Plus High-Content Screening System (PerkinElmer). Image analysis was conducted with MATLAB standard and custom-written scripts. For each cell, the integrated nuclear Hoechst signal and EdU positivity were used to determine cell cycle phase and values were averaged for each treatment.

\section{Apoptosis}

Live cell imaging for activated caspase-3/7 was performed using the IncuCyte ${ }^{\circledR}$ ZOOM (Essen Bioscience). Cells were seeded in 96 well plates and treated the next day with 100nM A947 and/or 1uM AMG-176 in the presence of Caspase-3/7 Green Detection Reagent (Essen Bioscience). Fluorescence was monitored over a 48h period, with data collection every $4 \mathrm{~h}$. Five planes of view were collected per well using 10x objective. Both phase contrast and green channel were collected for all wells. Data is presented as fluorescent events per well.

\section{RNA sequencing and analysis}

For in vitro gene expression studies, HCC2302 or HCC515 cells were treated with DMSO or A947(100nM) for both 24h and 96h prior to isolation of total RNA using the MagMax mirVana total RNA isolation kit (ThermoFisher Scientific, A27828). In addition, HCC2302-shNTC and HCC2302-shSMARCA2 cells were treated for 168h prior to isolation of RNA. For in vivo gene expression studies, total RNA was isolated from xenograft tissues as above. RNA concentrations were measured by NanoDrop8000 (ThermoFisher). Integrity of RNA was assessed by Bioanalyzer 2100 prior to library generation using 500ng RNA. Libraries were prepared using the TruSeq Stranaded Total RNA Library Prep Kit (Illumina), multiplexed and sequenced on Illumina HiSeq2500 (Illumina) to 
generate $\sim 30 \mathrm{M}$ single end, 50 base pair reads. Raw sequencing reads for in vitro samples were mapped to the UCSC human genome (GRCh38/hg38) using GSNAP software ${ }^{4}$. In order to remove potential mouse stromal contamination for in vivo xenograft samples, raw sequencing reads were stripped of reads showing complete alignment to the UCSC mouse genome (mm10) using Xenome software ${ }^{5}$. Remaining reads not showing complete alignment to $\mathrm{mm} 10$ were then mapped to GRCh38/hg38 using GSNAP software. Gene expression counts were obtained by quantifying the number of reads uniquely mapping to each gene locus. Lowly expressed genes were removed from all samples using a high-pass filter for genes with at least 15 counts in at least $10 \%$ of samples ( 6 of 54). Quantile normalized $\log _{2}$ counts per million (LogCPM) of sufficiently covered genes were generated using the voom function of the limma analysis pipeline ${ }^{6}$. Differential gene expression analysis was performed using edgeR ${ }^{7}$. Significantly downregulated or upregulated genes were defined by a $\log$ fold change $\left(\log _{2} \mathrm{FC}\right)$ absolute value $>1$ and a false discovery rate $(\mathrm{FDR})<0.05$. To evaluate in vivo samples, a consensus, putative SMARCA2 target gene set was defined by genes significantly downregulated by A947 treatment in both HCC515 and HCC2302 in vitro samples at both early ( 24 hours) and late ( 96 hours) time points.

\section{Mice}

Female Crl:NU-Foxn1nu (NU/NU Nude) or CB17/Icr-Prkdcscid/IcrIcoCrl (Fox Chase CB17) mice aged 6-8 weeks were purchased for Charles River laboratories. Mice received food and water ad libitum and were allowed to acclimate for 1-2 weeks before being used for experiments. All animal work was approved and conducted in accordance with the approval from the Institutional Animal Care and Use Committee (IACUC). All animal studies complied with the ethical regulations and humane endpoint criteria according to the NIH Guidelines for the Care and Use of Laboratory Animals.

\section{Xenograft seeding}

HCC515 and HCC2302 were propagated in RPMI-1640/10\%FBS (Gibco, \#61870-036), and Calu-6 in EMEM/10\%FBS (ATCC, \#30-2003). The cells were lifted with Trypsin (0.25\%) (Gibco, \#25200-056), spun down at 500xg, washed at least 3 times with DPBS (Gibco, \#14190-144), and the pellet resuspended in 50\%Matrigel (Corning, \#354234) / 50\% phenol-red free RPMI-1640 (Gibco, \#11835-030). Implant cell numbers for HCC515 and Calu- 6 was $5 \times 10^{\wedge} 6$ and for $\mathrm{HCC} 2032$ was $10 \times 10^{\wedge} 6$ in a 100 or $200 \mathrm{ul}$ volume.

\section{HCC2302, Calu-6, HCC515 xenograft mouse models}

Tumor cells were implanted into the right flank of the NU/NU or FOX CHASE SCID mice. The Tumor growth was monitored daily, and tumors were measured twice a week using digital calipers. Tumor volume was determined using the following formula (width $x$ width $x$ length)/2), where all measurements are in mm and the tumor volume is in $\mathrm{mm}^{3}$. The treatment started once the average tumor volume reached 150-200 $\mathrm{mm} 3$. Treatment was started approximately 3 weeks after cell implantation. The animals were randomly assigned into separate groups $(n=6-10$ animals per group) such that each group had nearly equal starting average tumor volume. Treatment groups were randomly assigned into groups treated with vehicle and A10947. A10947 was dosed 5mg per kg of body weight into the lateral tail vein intravenously once a week or every other week for tumor growth studies or only once for PK/PD studies. A10947 was formulated for intravenous dosing in 10\% Hydroxypropyl Beta Cyclodextrin (HP-b$\mathrm{CD})$ and $50 \mathrm{mM}$ sodium acetate in water $(\mathrm{pH} 4.0)$. All dosing solutions were filtered prior to injection using a 0.2micron filter to endure sterility. Mice were weighed twice a week, and dosing was performed the treatments were given according to the mouse's individual weight. Mice were euthanized using an IACUC approved method of euthanasia when an individual mouse reached a maximum tumor size humane endpoint, defined according to institutional policy concerning tumor endpoints in rodents. In addition, to prevent excessive pain or distress, the mice were euthanized if the tumors became ulcerated or if the mice showed any signs of ill health. Post euthanasia, blood and various tissues including tumors were collected for further analyses.

Analyses and comparisons of tumor growth were performed using a package of customized functions in R (Version 3.4.2 and 3.6.2 ; R Foundation for Statistical Computing; Vienna, Austria), which integrates software from open source packages (e.g., lme4, mgcv, gamm4, multcomp, settings, and plyr) and several packages from tidyverse (e.g., magrittr, dplyr, tidyr, and ggplot2) (Forrest et al. 2020). Briefly, as tumors generally exhibit exponential growth, tumor volumes were subjected to natural $\log$ transformations before analysis. Estimates of group-level efficacy were obtained by calculating percent tumor growth inhibition (TGI). This value represents the percent difference between the area under the curves (AUCs) of the treatment and reference group fits which are calculated after backtransforming tumor volumes to the original scale, correcting for starting tumor burden, and averaging over a 
common time period. Positive values indicate anti-tumor effects, with $100 \%$ denoting stasis and values $>100 \%$ denoting regression (negative values indicate a pro-tumor effect).

\section{Xenograft tissue processing for pharmacodynamic assessments}

Xenografts were harvested, divided into pieces, flash frozen and stored at -80C. For transcript-based pharmacodynamic readouts, RNA was isolated using the MagMAX mirVana total RNA isolation kit. For proteinbased pharmacodynamic readouts, RIPA + Halt protease inhibitor (Thermo Fisher, \#74830) was used at 400ul per tube, regardless of tumor weight. A steel ball was used in each sample in the TissueLyzer at $26 \mathrm{~Hz}$ for 4 minutes. The homogenization block was stopped half way through the process and the block flipped over for the duration of the process. Lysates were sonicated for 30 seconds on $20 \mathrm{~Hz}$ in an icy bath. The lysates were spun clean at 15,000 RPM for 15 minutes at 4C. Samples were then assessed for concentration by BCA at a dilution of 1:25. Western samples were prepared at 1ug/ul in SDS-PAGE loading buffer/denaturing agent (Life Technologies, \#NP0007 + \#B0009) and denatured at $95 \mathrm{C}$ for 5 minutes. Samples were used immediately or frozen at -20C until blotted.

\section{Immunoblotting (in vivo samples)}

Protein (8ug) was loaded on 4-15\% Criterion Tris/Glycine gels (Bio-Rad, \#5671085) and run for 60 minutes at 150 constant volts in $1 \mathrm{X}$ Tris/Glycine buffer (Bio-Rad, \#1610732). Protein was transferred from gels to nitrocellulose with Bio-Rad Turbo on default setting. All blots were air-dried, rehydrated with TBS and blocked for 1 hour at RT in 5\% BSA in TBST (0.1\%). Blots were exposed to primary antibody in 5\% BSA in TBST $(0.1 \%)$ overnight at $4 \mathrm{C}$. Blots were washed $3 \mathrm{x}$ with TBST $(0.1 \%), 5$ minutes per wash, at RT. Secondary antibody was added at 1:18,000: anti-rabbit-HRP (CST 7074) and/or anti-mouse-HRP (CST 7076) in 5\% BSA in TBST $(0.1 \%)$. Blots were incubated at RT for 1 hour. Blots were washed 3 times in TBST $(0.1 \%)$ for 5 minutes each wash at RT. All incubations and washing were done while rocking. Signal was developed with $6 \mathrm{ml}$ of Femto Max ECL substrate (ThermoFisher, \#34094) for 4 minutes and blots read on ChemiDoc. Densitometry was performed with ImageLab.

\section{Quantitative RT-PCR}

Gene expression levels were determined by Taqman using the following Taqman gene expression assays (KRT80, Hs01372363_g1; PLAU, Hs01547051_g1; GUSB, Hs00939627_m1) and the Taqman RNA-to-Ct 1-Step kit (ThermoFisher Scientific). Analysis is performed using QuantStudio ${ }^{\text {TM }} 7$ Flex Real-Time PCR System (ThermoFisher Scientific). Expression levels are normalized $\left(2^{-\Delta \mathrm{Ct}}\right)$ to the housekeeping gene, GUSB and presented relative to expression levels in vehicle-treated tumors.

\section{Pharmacokinetics assessment}

For Tumor: After the addition of $30 \mu \mathrm{L}$ of acetonitrile per $10 \mathrm{mg}$ of tumor, tumor samples were homogenized. 100 $\mu \mathrm{L}$ of tumor homogenate was pipetted out for analysis. After the addition of $50 \mu \mathrm{L}$ of DMSO:acetonitrile 1:1 (v/v), $20 \mu \mathrm{L}$ of $2 \mu \mathrm{g} / \mathrm{mL}$ propranolol in methanol:water 1:1 (v/v) as internal standard (IS), and $200 \mu \mathrm{L}$ of chilled acetonitrile, tumor samples were vortexed and centrifuged at $3500 \mathrm{rpm}$ for $30 \mathrm{~min}$. $2 \mu \mathrm{L}$ of supernatant was injected onto an AB Sciex API 4000 LC-MS/MS system coupled with a Shimadzu Prominence HPLC for analysis. For plasma: $20 \mu \mathrm{L}$ of DMSO:acetonitrile $1: 1(\mathrm{v} / \mathrm{v})$ and $20 \mu \mathrm{L}$ of $2 \mu \mathrm{g} / \mathrm{mL}$ propranolol in methanol:water 1:1 (v/v) as internal standard is added into $20 \mu \mathrm{L}$ of plasma sample, then $200 \mu \mathrm{L}$ of chilled acetonitrile was added to precipitate protein. Samples were vortexed and centrifuged at $3500 \mathrm{rpm}$ for $10 \mathrm{~min}$. $2 \mu \mathrm{L}$ of supernatant was injected onto an AB Sciex API 4000 LC-MS/MS system coupled with a Shimadzu Prominence HPLC for analysis. LC separation was performed on a Phenomenex Synergi Polar-RP column $(4 \mu \mathrm{m}, 80 \AA, 2 \times 50 \mathrm{~mm})$ with $0.1 \%$ acetic acid $1 \mathrm{mM}$ ammonium acetate in water as mobile phase $\mathrm{A}$ and $50 \mathrm{mM}$ acetic acid in acetonitrile as mobile phase $\mathrm{B}$. A gradient elution at $0.5 \mathrm{~mL} / \mathrm{min}$ started with $30 \% \mathrm{~B}$. B component was increased linearly to $75 \%$ in 0.5 minute. After holding at $75 \% \mathrm{~B}$ for 1.5 minutes, the column was reequilibrated with $30 \% \mathrm{~B}$ for 0.75 minute. Mass spectrometric detection was performed with TurboSpray ionization in positive ion mode.

\section{Drug combination screens}

A chemical library comprising 723 compounds arrayed in nine-point dose-response was screened in the absence or presence of a fixed dose of 100nM A947. Compounds were obtained from in-house synthesis or purchased from commercial vendors. Cells were dispensed using the Multidrop ${ }^{T M}$ Combi Reagent Dispenser (Thermo Scientific; Waltham, MA) into 384-well, black, clear-bottom plates (Corning, Tewksbury, MA) at seeding densities previous 
determined to achieve approximately $70-80 \%$ confluence at the final time point of the assay. Following overnight culture, compounds were dispensed using the Bravo Automated Liquid-Handling Platform (Agilent;

Santa Clara, CA). Following a 5 day culture period, $25 \mu \mathrm{L}$ CellTiter-Glo ${ }^{\circledR}$ reagent was added using a MultiFlo ${ }^{\mathrm{TM}}$ Microplate Dispenser (BioTek). Cell lysis was induced by mixing for 30 minutes on an orbital shaker prior to incubating plates at room temperature for 10 minutes to stabilize the luminescent signal. Luminescence was read by a 2104 EnVision ${ }^{\circledR}$ Multilabel Plate Reader (PerkinElmer; Waltham, MA). Data was processed using Genedata Screener ${ }^{\circledR}$, Version 15 (Genedata; Basel, Switzerland), with a four-parameter Hill equation using compound dose-response data normalized to the median of 42 vehicle-treated wells on each plate. A "Robust Fit" strategy was also employed by Genedata Screener ${ }^{\circledR}$, which is based on Tukey's biweight and is resistant to outlier data. The reported absolute $\mathrm{IC}_{50}$ is the dose at which cross-run estimated inhibition is $50 \%$ relative to DMSO control wells. Data is plotted as the difference in the $\mathrm{IC}_{50}$ in the presence versus absence of A947. For matrix-based combinations, cells were seeded and assessed for viability in the same manner as described for the chemical library screen. Cells were treated with A947 (top concentration, 5uM) in combination with AMG-176 (top concentration, $500 \mathrm{nM}$ ) or $\mathrm{S} 63845$ (top concentration, 500nM) in a 3-fold dilution, 9x9 matrix. Drug synergy was assessed using the Bliss independence mode $l^{8}$ and data is presented as excess matrix heatmaps that represent differences between the observed and predicted values determined from the Bliss model for each concentration pair.

1. Phu, L. et al. Dynamic Regulation of Mitochondrial Import by the Ubiquitin System. Mol Cell 77, 1107-1123.e10 (2020).

2. Rose, C. M. et al. Highly Multiplexed Quantitative Mass Spectrometry Analysis of Ubiquitylomes. Cell Syst 3, 395-403.e4 (2016).

3. Choi, M. et al. MSstats: an R package for statistical analysis of quantitative mass spectrometry-based proteomic experiments. Bioinformatics 30, 2524-2526 (2014).

4. Wu, T. D. \& Nacu, S. Fast and SNP-tolerant detection of complex variants and splicing in short reads.

Bioinformatics 26, 873-881 (2010).

5. Conway, T. et al. Xenome - a tool for classifying reads from xenograft samples. Bioinformatics 28, i172-i178 (2012).

6. Law, C. W., Chen, Y., Shi, W. \& Smyth, G. K. voom: precision weights unlock linear model analysis tools for RNA-seq read counts. Genome Biol 15, R29 (2014).

7. Robinson, M. D., McCarthy, D. J. \& Smyth, G. K. edgeR: a Bioconductor package for differential expression analysis of digital gene expression data. Bioinformatics 26, 139-140 (2010).

8. Bliss, C. I. The toxicity of poisons applied jointly. Annals of Applied Biology (1939) doi:https://doi.org/10.1111/j.1744-7348.1939.tb06990.x. 


\section{Chemical Synthesis}

All chemicals, reagents and solvents were at least reagent grade and obtained from various commercial sources. General removal of solvents was conducted by using a rotary evaporator. Residual solvents were then removed from the compounds using a vacuum manifold with $<10 \mathrm{~mm} \mathrm{Hg}$ pressure and/or via compound lyophilization. NMR spectra were recorded on a Bruker $400 \mathrm{MHz}$ spectrometer and are reported in parts per million (ppm) using residual nondeuterated solvents. LCMS spectral data was obtained using standard electrospray ionization mass spectrometry. Normal phase silica gel chromatography, flash column and preparatory thin-layer, was conducted on Merck silica gel 60, or as specifically indicated. Reverse phase and chiral chromatographic separations were conducted using columns and conditions specifically indicated in the experimental procedures below.

\section{4-((1r,3r)-3-(Benzyloxy)cyclobutoxy)pyridine (1)}

To a solution of pyridin-4-ol (3.20 g, $33.7 \mathrm{mmol}, 1.5 \mathrm{eq})$ and (1s,3s)-3-(benzyloxy)cyclobutan-1-ol (4 g, $22.4 \mathrm{mmol}$, $1 \mathrm{eq})$ in tetrahydrofuran $(200 \mathrm{~mL})$ was added triphenylphosphine $(7.06 \mathrm{~g}, 26.9 \mathrm{mmol}, 1.2 \mathrm{eq})$ and diisopropyl azodicarboxylate $(5.45 \mathrm{~g}, 26.9 \mathrm{mmol}, 1.2 \mathrm{eq})$ in one portion at $10^{\circ} \mathrm{C}$ under nitrogen. The mixture was stirred at 50 ${ }^{\circ} \mathrm{C}$ for 12 hours. The reaction mixture was concentrated under reduced pressure to remove tetrahydrofuran. Water $(50 \mathrm{~mL})$ was added, and the mixture was stirred for 1 minute. The aqueous phase was extracted with dichloromethane $(50 \mathrm{~mL} \times 3)$. The combined organic phase was washed with brine $(50 \mathrm{~mL} \times 2)$, dried with anhydrous sodium sulfate, filtered, and concentrated in vacuum. The residue was purified by silica gel column chromatography (petroleum ether: tetrahydrofuran from 20:1 to 5:1) and, additionally, by reverse phase C18 column chromatography [5-50\% acetonitrile: water $(0.5 \%$ ammonium hydroxide $)] .4-((1 \mathrm{r}, 3 \mathrm{r})-3-$

(Benzyloxy)cyclobutoxy)pyridine (3.2 g, $12.5 \mathrm{mmol}, 55 \%$ yield) was obtained as a white solid.

1-Benzyl-4-((1r,3r)-3-(benzyloxy)cyclobutoxy)pyridin-1-ium bromide (2)

To a solution of 4-((1r,3r)-3-(Benzyloxy)cyclobutoxy)pyridine $(4.2 \mathrm{~g}, 16.5 \mathrm{mmol}, 1 \mathrm{eq})$ in toluene $(65 \mathrm{~mL})$ was added benzyl bromide $(2.81 \mathrm{~g}, 16.5 \mathrm{mmol}, 1 \mathrm{eq})$. The mixture was stirred at $80^{\circ} \mathrm{C}$ for 12 hours. The reaction mixture was concentrated under reduced pressure to remove toluene. The crude product was triturated with petroleum ether $(80 \mathrm{~mL})$. 1-Benzyl-4-((1r,3r)-3-(benzyloxy)cyclobutoxy)pyridin-1-ium bromide $(6.5 \mathrm{~g}, 15.3 \mathrm{mmol}$, $92 \%$ yield) was obtained as a white solid.

1-Benzyl-4-((1r,3r)-3-(benzyloxy)cyclobutoxy)-1,2,3,6-tetrahydropyridine (3) To a solution of 1-benzyl-4-((1r,3r)-3-(benzyloxy)cyclobutoxy)pyridin-1-ium bromide (6.5 g, $15.3 \mathrm{mmol}, 1$ eq) in ethanol $(120 \mathrm{~mL})$ was added sodium borohydride $(3.46 \mathrm{~g}, 91.5 \mathrm{mmol}, 6 \mathrm{eq})$ at $0{ }^{\circ} \mathrm{C}$. The mixture was stirred at 15 ${ }^{\circ} \mathrm{C}$ for 4 hours. The reaction mixture was concentrated under reduced pressure to remove ethanol. The residue was diluted with water $(25 \mathrm{~mL})$ and extracted with ethyl acetate $(50 \mathrm{~mL} \times 2)$. The combined organic phase was washed with saturated brine $(40 \mathrm{~mL} \times 3)$, dried with anhydrous sodium sulfate, filtered, and concentrated in vacuum. 1Benzyl-4-((1r,3r)-3-(benzyloxy)cyclobutoxy)-1,2,3,6-tetrahydropyridine (4.5 g, $12.9 \mathrm{mmol}, 84 \%$ yield) was obtained as a colorless oil.

(1r,3r)-3-((1-Benzylpiperidin-4-yl)oxy)cyclobutan-1-ol (4)

To a solution of 1-benzyl-4-((1r,3r)-3-(benzyloxy)cyclobutoxy)-1,2,3,6-tetrahydropyridine (4.5 g, 12.9 mmol, 1 eq) in tetrahydrofuran $(95 \mathrm{~mL})$ and ethanol $(70 \mathrm{~mL})$ was added $10 \%$ palladium on activated carbon catalyst $(0.5 \mathrm{~g})$ under nitrogen atmosphere. The suspension was degassed and purged with hydrogen three times. The mixture was stirred under hydrogen pressure $(50 \mathrm{psi})$ at $35^{\circ} \mathrm{C}$ for 12 hours. The reaction mixture was filtered, and the filtrate was concentrated. The residue was purified by silica gel column chromatography (dichloromethane: methanol:

ammonium hydroxide from 20:1:0 to 10:1:0.1). (1r,3r)-3-((1-Benzylpiperidin-4-yl)oxy)cyclobutan-1-ol (2.8 g, 10.71 $\mathrm{mmol}, 83 \%$ yield) was obtained as a colorless oil.

tert-Butyl 4-((1r,3r)-3-hydroxycyclobutoxy)piperidine-1-carboxylate (5)

To a solution of (1r,3r)-3-((1-benzylpiperidin-4-yl)oxy)cyclobutan-1-ol (1.1 g, $4.21 \mathrm{mmol}, 1 \mathrm{eq})$ in methanol (10 $\mathrm{mL}$ ) was added $10 \%$ palladium hydroxide on activated carbon catalyst $(591 \mathrm{mg})$ and di-tert-butyl dicarbonate $(1.84$ $\mathrm{g}, 8.42 \mathrm{mmol}, 2 \mathrm{eq}$ ) under nitrogen atmosphere. The suspension was degassed and purged with hydrogen three times. The mixture was stirred under hydrogen pressure $(50 \mathrm{psi})$ at $25^{\circ} \mathrm{C}$ for 12 hours. The reaction mixture was filtered, and the filtrate was concentrated. The residue was purified by silica gel chromatography (petroleum ether: ethyl acetate from 20:1 to 2:1). tert-Butyl 4-((1r,3r)-3-hydroxycyclobutoxy)piperidine-1-carboxylate (820 mg, 3.02 mmol, $71 \%$ yield) was obtained as a colorless oil. 
tert-Butyl 4-((1r,3r)-3-((4-bromopyridin-2-yl)oxy)cyclobutoxy)piperidine-1-carboxylate (6)

A mixture of tert-butyl 4-((1r,3r)-3-hydroxycyclobutoxy)piperidine-1-carboxylate (1 g, $3.69 \mathrm{mmol}, 1 \mathrm{eq})$, 4-bromo2-fluoro-pyridine ( $778 \mathrm{mg}, 4.42 \mathrm{mmol}, 1.2 \mathrm{eq})$, and cesium carbonate $(2.40 \mathrm{~g}, 7.37 \mathrm{mmol}, 2 \mathrm{eq})$ in acetonitrile (10 $\mathrm{mL}$ ) was degassed and purged with nitrogen three times. The mixture was then stirred at $90{ }^{\circ} \mathrm{C}$ for 12 hours under the nitrogen atmosphere. The reaction mixture was filtered and concentrated. The crude product was purified by silica gel chromatography (petroleum ether: ethyl acetate from 1:0 to 10:1). tert-Butyl 4-((1r,3r)-3-((4bromopyridin-2-yl)oxy)cyclobutoxy)piperidine-1-carboxylate (1.36 g, $3.18 \mathrm{mmol}, 86 \%$ yield) was obtained as colorless oil.

Benzyl 8-(2-((1r,3r)-3-((1-(tert-butoxycarbonyl)piperidin-4-yl)oxy)cyclobutoxy)pyridin-4-yl)-3,8diazabicyclo[3.2.1] octane-3-carboxylate (7)

A mixture of benzyl 3,8-diazabicyclo[3.2.1]octane-3-carboxylate (784 mg, $3.18 \mathrm{mmol}, 1 \mathrm{eq})$, tert-butyl 4-((1r,3r)-3((4-bromopyridin-2-yl)oxy)cyclobutoxy)piperidine-1-carboxylate (1.36 g, $3.18 \mathrm{mmol}, 1 \mathrm{eq})$, chloro(2dicyclohexylphosphino-2',6'-diisopropoxy-1,1'-biphenyl)[2-(2'-amino-1,1'-biphenyl)]palladium(II) [RuPhos-PdG2] (148 $\mathrm{mg}, 0.19 \mathrm{mmol}, 0.06 \mathrm{eq})$, and cesium carbonate $(2.07 \mathrm{~g}, 6.37 \mathrm{mmol}, 2 \mathrm{eq})$ in toluene $(27 \mathrm{~mL})$ was degassed and purged with nitrogen three times. The mixture was then stirred at $110^{\circ} \mathrm{C}$ for $12 \mathrm{~h}$ under the nitrogen atmosphere. Water $(30 \mathrm{~mL})$ was added, and the mixture was extracted with ethyl acetate $(40 \mathrm{~mL} \times 2)$. The combined organic layers were washed with saturated aqueous sodium chloride $(30 \mathrm{~mL}$ x 2), dried over anhydrous sodium sulfate, filtered and concentrated under reduced pressure. The crude product was purified by silica gel chromatography (petroleum ether: ethyl acetate from 1:0 to 1:1). Benzyl 8-(2-((1r,3r)-3-((1-(tertbutoxycarbonyl)piperidin-4-yl)oxy)cyclobutoxy)pyridin-4-yl)-3,8-diazabicyclo[3.2.1]octane-3-carboxylate (1.35 g, $2.28 \mathrm{mmol}, 71 \%$ yield) was obtained as a colorless oil.

tert-Butyl 4-((1r,3r)-3-((4-(3,8-diazabicyclo[3.2.1]octan-8-yl)pyridin-2-yl)oxy)cyclobutoxy)piperidine-1carboxylate (8)

To a solution of benzyl 8-(2-((1r,3r)-3-((1-(tert-butoxycarbonyl)piperidin-4-yl)oxy)cyclobutoxy)pyridin-4-yl)-3,8diazabicyclo[3.2.1] octane-3-carboxylate $(1.35 \mathrm{~g}, 2.28 \mathrm{mmol}, 1 \mathrm{eq})$ in a mixture of tetrahydrofuran $(27 \mathrm{~mL})$ and ethanol $(27 \mathrm{~mL})$ was added $10 \%$ palladium hydroxide on activated carbon catalyst $(320 \mathrm{mg})$ under nitrogen. The suspension was degassed under vacuum and purged with hydrogen several times. The mixture was stirred under hydrogen pressure $(50 \mathrm{psi})$ at $60^{\circ} \mathrm{C}$ for 12 hours. The reaction mixture was filtered, and the filtrate was concentrated. tert-Butyl 4-((1r,3r)-3-((4-(3,8-diazabicyclo[3.2.1]octan-8-yl)pyridin-2yl)oxy)cyclobutoxy)piperidine-1-carboxylate ( $932 \mathrm{mg}, 2.03 \mathrm{mmol}, 89 \%$ yield) was obtained as a brown solid.

tert-Butyl 4-((1r,3r)-3-((4-(3-(3-amino-6-chloropyridazin-4-yl)-3,8-diazabicyclo[3.2.1]octan-8-yl)pyridin-2yl)oxy)cyclobutoxy)piperidine-1-carboxylate (9)

A mixture of tert-butyl 4-((1r,3r)-3-((4-(3,8-diazabicyclo[3.2.1]octan-8-yl)pyridin-2-yl)oxy)cyclobutoxy)piperidine1-carboxylate ( $932 \mathrm{mg}, 2.03 \mathrm{mmol}, 1 \mathrm{eq}$ ), 4-bromo-6-chloro-pyridazin-3-amine (508 $\mathrm{mg}, 2.44 \mathrm{mmol}, 1.2 \mathrm{eq}$ ), and $N, N$-diisopropylethylamine $(2.63 \mathrm{~g}, 20.3 \mathrm{mmol}, 3.54 \mathrm{~mL}, 10 \mathrm{eq})$ in dimethylsulfoxide $(30 \mathrm{~mL})$ was degassed and purged with nitrogen three times. The mixture was then stirred at $130^{\circ} \mathrm{C}$ for $3 \mathrm{~h}$ under the nitrogen atmosphere. Water $(30 \mathrm{~mL})$ was added, and the mixture was extracted with ethyl acetate $(30 \mathrm{~mL} \times 2)$. The combined organic layers were washed saturated aqueous sodium chloride $(30 \mathrm{~mL}$ x 2$)$, dried over anhydrous sodium sulfate and concentrated. The residue was purified by semi-preparative reverse phase HPLC (column: Phenomenex Gemini C18 250*50 10 um; mobile phase: [water (0.05\% ammonia hydroxide v/v)-ACN]; B\%: 45\%-70\%). tert-Butyl 4((1r,3r)-3-((4-(3-(3-amino-6-chloropyridazin-4-yl)-3,8-diazabicyclo[3.2.1]octan-8-yl)pyridin-2-

yl)oxy)cyclobutoxy)piperidine-1-carboxylate ( $772 \mathrm{mg}, 1.32 \mathrm{mmol}, 64 \%$ yield) was obtained as a yellow oil.

tert-Butyl 4-((1r,3r)-3-((4-(3-(3-amino-6-(2-hydroxyphenyl)pyridazin-4-yl)-3,8-diazabicyclo[3.2.1]octan-8yl)pyridin-2-yl)oxy)cyclobutoxy)piperidine-1-carboxylate (10)

A mixture of tert-butyl 4-((1r,3r)-3-((4-(3-(3-amino-6-chloropyridazin-4-yl)-3,8-diazabicyclo[3.2.1]octan-8yl)pyridin-2-yl)oxy)cyclobutoxy)piperidine-1-carboxylate (772 mg, $1.32 \mathrm{mmol}, 1$ eq), (2-hydroxyphenyl)boronic acid (218 mg, $1.58 \mathrm{mmol}, 1.2 \mathrm{eq})$, tetrakis[triphenylphosphine]palladium(0) (152 mg, $0.13 \mathrm{mmol}, 0.1 \mathrm{eq})$, and potassium carbonate $(364 \mathrm{mg}, 2.63 \mathrm{mmol}, 2 \mathrm{eq})$ in a mixture of dioxane $(12 \mathrm{~mL})$ and water $(2 \mathrm{~mL})$ was degassed and purged with nitrogen three times. The mixture was then stirred at $90^{\circ} \mathrm{C}$ for $10 \mathrm{~h}$ under the nitrogen atmosphere. The reaction mixture was concentrated. The residue was purified by semi-preparative reverse phase HPLC (column: Kromasil 250*50 mm*10 um; mobile phase: [water (0.1\%trifluoroacetic acid)-ACN]; B\%: 20\%-50\%). tert-Butyl 4- 
((1r,3r)-3-((4-(3-(3-amino-6-(2-hydroxyphenyl)pyridazin-4-yl)-3,8-diazabicyclo[3.2.1]octan-8-yl)pyridin-2yl)oxy)cyclobutoxy)piperidine-1-carboxylate $(500 \mathrm{mg}, 0.71 \mathrm{mmol}, 54 \%$ yield) was obtained as a yellow solid.

2-(6-amino-5-(8-(2-((1r,3r)-3-(piperidin-4-yloxy)cyclobutoxy)pyridin-4-yl)-3,8-diazabicyclo[3.2.1]octan-3yl)pyridazin-3-yl)phenol (11)

To a solution of tert-butyl 4-((1r,3r)-3-((4-(3-(3-amino-6-(2-hydroxyphenyl)pyridazin-4-yl)-3,8-

diazabicyclo[3.2.1] octan-8-yl)pyridin-2-yl)oxy)cyclobutoxy)piperidine-1-carboxylate (500 $\mathrm{mg}, 0.78 \mathrm{mmol}, 1 \mathrm{eq}$ ) in dichloromethane $(5 \mathrm{~mL})$ was added hydrochloric acid/dioxane $(4 \mathrm{M}, 5 \mathrm{~mL}, 25.8 \mathrm{eq})$. The mixture was stirred at 20 ${ }^{\circ} \mathrm{C}$ for 2 hours. The mixture was concentrated, and crude 2-(6-amino-5-(8-(2-((1r,3r)-3-(piperidin-4-

yloxy)cyclobutoxy)pyridin-4-yl)-3,8-diazabicyclo[3.2.1]octan-3-yl)pyridazin-3-yl)phenol trihydrochloride (500 mg) was obtained as a yellow solid.

Methyl 2-(3-hydroxyisoxazol-5-yl)-3-methyl-butanoate (12)

To a solution of 2-(3-hydroxyisoxazol-5-yl)-3-methyl-butanoic acid ${ }^{1}(1 \mathrm{~g}, 5.40 \mathrm{mmol}, 1 \mathrm{eq})$ in methanol (10 mL) was added thionyl chloride $(2.57 \mathrm{~g}, 21 \mathrm{mmol}, 1.57 \mathrm{~mL}, 4 \mathrm{eq})$ at $0^{\circ} \mathrm{C}$. The reaction mixture was stirred at $70{ }^{\circ} \mathrm{C}$ for 3 hours. The reaction mixture was concentrated under reduced pressure. The residue was diluted with water (50 ml) and extracted with ethyl acetate $(30 \mathrm{~mL} \times 3)$. The combined organic layers were washed with brine $(80 \mathrm{~mL} \times 2)$, dried over anhydrous sodium sulfate, filtered, and concentrated under reduced pressure. Methyl 2-(3hydroxyisoxazol-5-yl)-3-methyl-butanoate ( $\mathrm{g}, 5.02 \mathrm{mmol}, 92 \%$ yield) was obtained as a yellow oil.

Methyl 3-methyl-2-[3-(1,1,2,2,3,3,4,4,4-nonafluorobutylsulfonyloxy)isoxazol-5-yl]butanoate (13)

To a solution of methyl 2-(3-hydroxyisoxazol-5-yl)-3-methyl-butanoate ( $800 \mathrm{mg}, 4.02 \mathrm{mmol}, 1 \mathrm{eq}$ ) in acetonitrile (5 $\mathrm{mL}$ ) was added potassium carbonate $(1.11 \mathrm{~g}, 8.03 \mathrm{mmol}, 2 \mathrm{eq})$ and perfluorobutyl sulfonyl fluoride $(1.46 \mathrm{~g}, 4.82$ $\mathrm{mmol}, 1.2 \mathrm{eq}$ ). The reaction mixture was stirred at $25^{\circ} \mathrm{C}$ for 12 hours. The reaction mixture was diluted with water $(50 \mathrm{~mL})$ and extracted with ethyl acetate $(30 \mathrm{~mL} \times 3)$. The combined organic layers were washed with brine $(80 \mathrm{~mL}$ x 3), dried over anhydrous sodium sulfate, filtered, and concentrated under reduced pressure. The residue was purified by silica gel column chromatography (petroleum ether/ethyl acetate from 100:1 to 20:1). Methyl 3-methyl2-[3-(1,1,2,2,3,3,4,4,4-nonafluorobutylsulfonyloxy)isoxazol-5-yl]butanoate (530 mg, $1.10 \mathrm{mmol}, 27 \%$ yield) was obtained as a colorless oil.

Methyl 2-[3-[4-(dimethoxymethyl)-1-piperidyl] isoxazol-5-yl]-3-methyl-butanoate (14)

To a mixture of methyl 3-methyl-2-[3-(1,1,2,2,3,3,4,4,4-nonafluorobutylsulfonyloxy)isoxazol -5-yl]butanoate (5 g, $10.39 \mathrm{mmol}, 1 \mathrm{eq}$ ) and 4-(dimethoxymethyl)piperidine (4.14 g, $26.0 \mathrm{mmol}, 2.5 \mathrm{eq}$ ) in $\mathrm{N}, \mathrm{N}$-dimethylformamide (50 $\mathrm{mL}$ ) was added $N, N$-diisopropylethylamine $(4.03 \mathrm{~g}, 31.2 \mathrm{mmol}, 5.4 \mathrm{~mL}, 3 \mathrm{eq})$ at $25^{\circ} \mathrm{C}$ in one portion under nitrogen. The mixture was stirred at $80{ }^{\circ} \mathrm{C}$ for 2 hours. The mixture was cooled to $25^{\circ} \mathrm{C}$ and poured into ice-water $(\mathrm{w} / \mathrm{w}=1 / 1)(30 \mathrm{~mL})$ and stirred for $15 \mathrm{~min}$. The aqueous phase was extracted with ethyl acetate $(50 \mathrm{~mL} \times 3)$. The combined organic phase was washed with brine $(50 \mathrm{~mL} \times 3)$, dried with anhydrous sodium sulfate, filtered, and concentrated in vacuum. The residue was purified by prep thin-layer chromatography (TLC) (petroleum ether: ethyl acetate $=3: 1)$ to afford methyl 2-[3-[4-(dimethoxymethyl)-1-piperidyl] isoxazol-5-yl]-3-methyl-butanoate $(0.354 \mathrm{~g}$, $1.04 \mathrm{mmol}, 10 \%$ yield) as a yellow oil.

2-[3-[4-(Dimethoxymethyl)-1-piperidyl]isoxazol-5-yl]-3-methyl- butanoic acid (15)

To a solution of methyl 2-[3-[4-(dimethoxymethyl)-1-piperidyl]isoxazol-5-yl]-3-methyl- butanoate (350 mg, 1.03 mmol, $1 e q)$ in tetrahydrofuran $(1 \mathrm{~mL})$ and methanol $(1 \mathrm{~mL})$ was added lithium hydroxide $(2 \mathrm{M}, 1 \mathrm{~mL}, 1.95 e q)$ in one portion under nitrogen. The mixture was stirred at $25^{\circ} \mathrm{C}$ for $1 \mathrm{~h}$. The $\mathrm{pH}$ of the reaction mixture was adjusted to 5-6 with trifluoroacetic acid, and the mixture was concentrated under reduced pressure at $45^{\circ} \mathrm{C}$. The residue was purified by preparative High Performance Liquid chromatography (HPLC) (column: Phenomenex Synergi C18 $150 * 25 * 10 u m$; mobile phase: [water( $0.225 \% \mathrm{FA}$ )-ACN];B\%: 40\%-70\%) to afford 2-[3-[4-(dimethoxymethyl)-1piperidyl]isoxazol-5-yl]-3-methyl-butanoic acid (190 mg, $0.58 \mathrm{mmol}, 56 \%$ yield) as a yellow oil.

tert-Butyl N-[(1S)-1-(4-bromophenyl)ethyl]carbamate (16)

To a solution of (1S)-1-(4-bromophenyl)ethanamine (24.9 g, $124.5 \mathrm{mmol}, 1 \mathrm{eq})$ in tetrahydrofuran $(350 \mathrm{~mL})$ was added triethylamine $(37.8 \mathrm{~g}, 373.4 \mathrm{mmol}, 3 \mathrm{eq})$ followed by di-tert-butyl dicarbonate $(28.5 \mathrm{~g}, 130.7 \mathrm{mmol}, 30 \mathrm{~mL}$, 
$1.05 \mathrm{eq}$ ) dropwise at $0{ }^{\circ} \mathrm{C}$ under nitrogen. The mixture was then stirred at $25{ }^{\circ} \mathrm{C}$ for 12 hours. The reaction mixture was concentrated under reduced pressure to remove tetrahydrofuran. Water $(400 \mathrm{~mL})$ was added, and the mixture was stirred for 1 minute. The aqueous phase was extracted with ethyl acetate $(200 \mathrm{~mL}$ x 3$)$. The combined organic phase was washed with brine $(200 \mathrm{~mL} \times 2)$, dried over anhydrous sodium sulfate, filtered, and concentrated in vacuum. The crude product was triturated with petroleum ether $(250 \mathrm{~mL})$. Compound tert-butyl N-[(1S)-1-(4bromophenyl)ethyl]carbamate (34.5 g, $114.93 \mathrm{mmol}, 92 \%$ yield) was obtained as a white solid.

tert-Butyl N-[(1S)-1-[4- (4-methylthiazol-5-yl)phenyl]ethyl]carbamate (17)

To a solution of tert-butyl N-[(1S)-1-(4-bromophenyl)ethyl]carbamate (14.5 g, $48.30 \mathrm{mmol}, 1 \mathrm{eq}$ ) and 4methylthiazole $(7.18 \mathrm{~g}, 72.45 \mathrm{mmol}, 1.5 \mathrm{eq})$ in dimethylacetamide $(15 \mathrm{~mL})$ was added palladium(II) acetate (542 $\mathrm{mg}, 2.42 \mathrm{mmol}, 0.05 \mathrm{eq})$ and potassium acetate $(9.48 \mathrm{~g}, 96.61 \mathrm{mmol}, 2 \mathrm{eq})$. The mixture was stirred at $90{ }^{\circ} \mathrm{C}$ for 12 h. Water $(300 \mathrm{~mL})$ was added, and the mixture was stirred for 1 minute. The aqueous phase was extracted with ethyl acetate $(100 \mathrm{~mL} \times 3)$. The combined organic phase was washed with brine $(100 \mathrm{~mL} \times 2)$, dried with anhydrous sodium sulfate, filtered, and concentrated in vacuum. The residue was purified by reverse phase $\mathrm{C} 18$ column chromatography [ACN/ H2O (0.5\% FA) from 5\% to 50\%]. tert-Butyl N-[(1S)-1-[4- (4-methylthiazol-5yl)phenyl]ethyl]carbamate $(9.8 \mathrm{~g}, 29.85 \mathrm{mmol}, 61 \%$ yield) was obtained as a gray solid.

(1S)-1-[4-(4-Methylthiazol-5-yl)phenyl]ethanamine (18)

To a solution of tert-butyl N-[(1S)-1-[4-(4-methylthiazol-5-yl)phenyl]ethyl]carbamate (1.5 g, $4.71 \mathrm{mmol}, 1 \mathrm{eq})$ in dichloromethane $(20 \mathrm{~mL})$ was added hydrochloride acid/dioxane $(4 \mathrm{M}, 20 \mathrm{~mL}, 17 \mathrm{eq})$. The mixture was stirred at 25 ${ }^{\circ} \mathrm{C}$ for 12 hours. The reaction mixture was concentrated under reduced pressure to remove dichloromethane. The crude product was triturated with petroleum ether (100 mL). Crude (1S)-1-[4-(4-methylthiazol-5yl)phenyl]ethanamine hydrochloride $(1.1 \mathrm{~g})$ was obtained as a yellow solid.

tert-Butyl (2S,4R)-4-hydroxy-2-[[(1S)-1-[4-(4-methylthiazol-5-yl)phenyl]ethyl]carbamoyl] pyrrolidine-1carboxylate (19)

To a solution of (2S,4R)-1-tert-butoxycarbonyl-4-hydroxy-pyrrolidine-2-carboxylic acid (998 mg, $4.32 \mathrm{mmol}, 1.1$ $e q)$ and $\mathrm{O}-(7$-azabenzotriazol-1-yl)-N,N,N',N'-tetramethyluronium hexafluorophosphate (1.79 g, $4.71 \mathrm{mmol}, 1.2 \mathrm{eq})$ in dimethylformamide $(10 \mathrm{~mL})$ were added $(1 \mathrm{~S})-1$-[4-(4-methylthiazol-5-yl)phenyl]ethanamine hydrochloride $(1 \mathrm{~g}$, $3.92 \mathrm{mmol}, 1 \mathrm{eq})$ and diisopropylethyl amine $(1.52 \mathrm{~g}, 11.77 \mathrm{mmol}, 2.05 \mathrm{~mL}, 3 \mathrm{eq})$. The reaction mixture was stirred at $15^{\circ} \mathrm{C}$ for 0.5 hour. The reaction mixture was poured into water $(20 \mathrm{~mL})$ and extracted with ethyl acetate $(30 \mathrm{~mL} \mathrm{x}$ $3)$. The combined organic layers were washed with brine $(50 \mathrm{~mL} \times 3)$, dried over anhydrous sodium sulfate, filtered, and concentrated under reduced pressure. The residue was purified by silica gel column chromatography (petroleum ether: ethyl acetate from 100:1 to 30:1). tert-Butyl (2S,4R)-4-hydroxy-2-[[(1S)-1-[4-(4-methylthiazol-5-

yl)phenyl]ethyl]carbamoyl] pyrrolidine-1-carboxylate (1.2 g, $2.78 \mathrm{mmol}, 70 \%$ yield) was obtained as a white solid.

(2S,4R)-4-hydroxy-N-[(1S)-1-[4-(4-methylthiazol-5-yl)phenyl]ethyl]pyrrolidine-2-carboxamide (20)

To a solution of tert-butyl (2S,4R)-4-hydroxy-2-[[(1S)-1-[4-(4-methylthiazol-5-yl)

phenyl]ethyl]carbamoyl]pyrrolidine-1-carboxylate $(1 \mathrm{~g}, 2.32 \mathrm{mmol}, 1 \mathrm{eq})$ in dichloromethane $(10 \mathrm{~mL})$ was added hydrochloric acid $(2.5 \mathrm{M}$ in dioxane, $5 \mathrm{~mL}, 5.4 \mathrm{eq})$. The reaction mixture was stirred at $15^{\circ} \mathrm{C}$ for 0.5 hour. The reaction mixture was concentrated under reduced pressure. (2S,4R)-4-hydroxy-N-[(1S)-1-[4-(4-methylthiazol-5yl)phenyl]ethyl]pyrrolidine-2-carboxamide hydrochloride ( $800 \mathrm{mg}, 2.17 \mathrm{mmol}, 93 \%$ yield) was obtained as a colorless oil.

(2S,4R)-1-[2-[3-[4-(dimethoxymethyl)-1-piperidyl]isoxazol-5-yl]-3-methyl-butanoyl]-4-hydroxy-N-[(1S)-1-[4-(4methylthiazol-5-yl)phenyl]ethyl]pyrrolidine-2-carboxamide (21)

To a mixture of 2-[3-[4-(dimethoxymethyl)-1-piperidyl]isoxazol-5-yl]-3-methyl-butanoic acid (190 mg, $0.58 \mathrm{mmol}$, $1 e q), N, N$-diisopropylethylamine $(225 \mathrm{mg}, 1.75 \mathrm{mmol}, 0.3 \mathrm{~mL}, 3 \mathrm{eq})$ and (2S,4R)-4-hydroxy-N-[(1S)-1-[4-(4methylthiazol-5-yl)phenyl]ethyl]pyrrolidine-2-carboxamide hydrochloride $(257 \mathrm{mg}, 0.70 \mathrm{mmol}, 1.2 \mathrm{eq})$ in $\mathrm{N}, \mathrm{N}$ - 
dimethylformamide $(10 \mathrm{~mL})$ was added O-(7-azabenzotriazol-1-yl)-N,N,N',N'-tetramethyluronium hexafluorophosphate $(288 \mathrm{mg}, 0.76 \mathrm{mmol}, 1.3 \mathrm{eq})$ in one portion at $0{ }^{\circ} \mathrm{C}$ under nitrogen. The mixture was stirred at $25^{\circ} \mathrm{C}$ for $1 \mathrm{~h}$. The mixture was poured into ice-water $(\mathrm{w} / \mathrm{w}=1 / 1)(30 \mathrm{~mL})$ and stirred for $15 \mathrm{~min}$. The aqueous phase was extracted with ethyl acetate $(30 \mathrm{~mL} x 3)$. The combined organic phase was washed with brine $(30 \mathrm{~mL} x$ 3 ), dried with sodium sulfate, filtered, and concentrated in vacuum. The residue was purified by silica gel chromatography (dichloromethane: methanol = 10:1) to afford (2S,4R)-1-[2-[3-[4-(dimethoxymethyl)-1piperidyl]isoxazol-5-yl]-3-methyl-butanoyl]-4-hydroxy-N-[(1S)-1-[4-(4-methylthiazol-5yl)phenyl]ethyl]pyrrolidine-2-carboxamide (208 $\mathrm{mg}, 0.32 \mathrm{mmol}, 55 \%$ yield) as a yellow oil.

(2S,4R)-1-[(2S)-2-[3-[4-(dimethoxymethyl)-1-piperidyl]isoxazol-5-yl]-3-methyl-butanoyl]-4-hydroxy-N-[(1S)-1-[4(4-methylthiazol-5-yl)phenyl]ethyl]pyrrolidine-2-carboxamide (22) and (2S,4R)-1-[(2R)-2-[3-[4-

(dimethoxymethyl)-1-piperidyl]isoxazol-5-yl]-3-methyl-butanoyl]-4-hydroxy-N-[(1S)-1-[4-(4-methylthiazol-5yl)phenyl]ethyl]pyrrolidine-2-carboxamide (23)

(2S,4R)-1-[2-[3-[4-(dimethoxymethyl)-1-piperidyl]isoxazol-5-yl]-3-methyl-butanoyl]-4-hydroxy-N-[(1S)-1-[4-(4methylthiazol-5-yl)phenyl]ethyl]pyrrolidine-2-carboxamide $(200 \mathrm{mg}, 0.31 \mathrm{mmol}, 1 \mathrm{eq})$ was purified by chiral SFC (column: DAICEL CHIRALPAK AD $(250 \mathrm{~mm} * 30 \mathrm{~mm}, 10 \mathrm{um})$; mobile phase: [0.1\%NH4OH/ IPA]; B\%: $45 \%)$ to produce (2S,4R)-1-[(2S)-2-[3-[4-(dimethoxymethyl)-1-piperidyl]isoxazol-5-yl]-3-methyl-butanoyl]-4-hydroxy-N[(1S)-1-[4-(4-methylthiazol-5-yl)phenyl]ethyl]pyrrolidine-2-carboxamide ( $84 \mathrm{mg}, 0.12 \mathrm{mmol}, 42 \%$ yield) (analytical retention time $1.88 \mathrm{~min}$ ) as a yellow oil and (2S,4R)-1-[(2R)-2-[3-[4-(dimethoxymethyl)-1piperidyl]isoxazol-5-yl]-3-methyl-butanoyl]-4-hydroxy-N-[(1S)-1-[4-(4-methylthiazol-5yl)phenyl]ethyl]pyrrolidine-2-carboxamide ( $90 \mathrm{mg}, 0.13 \mathrm{mmol}, 45 \%$ yield) (analytical retention time $2.23 \mathrm{~min}$ ) as a yellow oil.

(2S,4R)-1-[(2R)-2-[3-(4-formyl-1-piperidyl)isoxazol-5-yl]-3-methyl-butanoyl]-4-hydroxy-N-[(1S)-1-[4-(4methylthiazol-5-yl)phenyl]ethyl]pyrrolidine-2-carboxamide (24)

To a solution of (2S,4R)-1-[(2R)-2-[3-[4-(dimethoxymethyl)-1-piperidyl]isoxazol-5-yl]-3- methyl-butanoyl]-4hydroxy-N-[(1S)-1-[4-(4-methylthiazol-5-yl)phenyl]ethyl]pyrrolidine-2-carboxamide (80 $\mathrm{mg}, 0.13 \mathrm{mmol}, 1 \mathrm{eq})$ in tetrahydrofuran $(2 \mathrm{~mL})$ was added sulfuric acid solution $(1 \mathrm{M}, 2 \mathrm{~mL}, 16 e q)$. The reaction mixture was stirred at 50 ${ }^{\circ} \mathrm{C}$ for 5 hours. To the mixture was added saturated sodium bicarbonate solution $(10 \mathrm{~mL})$, and the mixture was extracted with ethyl acetate $(20 \mathrm{~mL} \times 3)$. The combined organic phase was washed with brine $(30 \mathrm{~mL})$, dried over sodium sulfate, filtered, and concentrated in vacuum. The crude (2S,4R)-1-[(2R)-2-[3-(4-formyl-1piperidyl)isoxazol-5-yl]-3-methyl-butanoyl]-4-hydroxy-N-[(1S)-1-[4-(4-methylthiazol-5yl)phenyl]ethyl]pyrrolidine-2-carboxamide ( $68 \mathrm{mg}, 0.11 \mathrm{mmol}$, 91\% yield) was obtained as a light yellow solid and used directly in the next step.

(2S,4R)-1-((2R)-2-(3-(4-((4-((1r,3r)-3-((4-(3-(3-Amino-6-(2-hydroxyphenyl)pyridazin-4-yl)-3,8diazabicyclo[3.2.1]octan-8-yl)pyridin-2-yl)oxy)cyclobutoxy)piperidin-1-yl)methyl)piperidin-1-yl)isoxazol-5-yl)-3methylbutanoyl)-4-hydroxy-N-((S)-1-(4-(4-methylthiazol-5-yl)phenyl)ethyl)pyrrolidine-2-carboxamide (A947) To a solution of 2-(6-amino-5-(8-(2-((1r,3r)-3-(piperidin-4-yloxy)cyclobutoxy)pyridin-4-yl)-3,8diazabicyclo[3.2.1] octan-3-yl)pyridazin-3-yl)phenol hydrochloride (365 mg, $0.59 \mathrm{mmol}, 1$ eq) in methanol $(20 \mathrm{~mL})$ was added sodium acetate (145 mg, $1.77 \mathrm{mmol}, 3 \mathrm{eq}$ ). To the mixture were then added (2S,4R)-1-[(2R)-2-[3-(4formyl-1-piperidyl)isoxazol- 5-yl]-3-methyl-butanoyl]-4-hydroxy-N-[(1S)-1-[4-(4-methylthiazol-5-

yl)phenyl]ethyl]pyrrolidine-2-carboxamide ( $350 \mathrm{mg}, 0.59 \mathrm{mmol}, 1 \mathrm{eq}$ ) and acetic acid (4 $\mathrm{mg}, 0.06 \mathrm{mmol}, 0.1 \mathrm{eq}$ ). The mixture was stirred at $25^{\circ} \mathrm{C}$ for 0.5 hour, after which sodium cyanoborohydride $(185 \mathrm{mg}, 2.95 \mathrm{mmol}, 5 \mathrm{eq})$ was added. The mixture was stirred at $25^{\circ} \mathrm{C}$ for 2 hours. The reaction mixture was concentrated under reduced pressure. The residue was purified by prep-HPLC (column: Phenomenex Synergi Max-RP 150*50 mm*10 um; mobile phase: [water $(0.2 \% \mathrm{FA})$ - ACN]; B\%: 80\% - 38\%). (2S,4R)-1-((2R)-2-(3-(4-((4-((1r,3r)-3-((4-(3-(3-Amino-6-(2hydroxyphenyl)pyridazin-4-yl)-3,8-diazabicyclo[3.2.1]octan-8-yl)pyridin-2-yl)oxy)cyclobutoxy)piperidin-1yl)methyl)piperidin-1-yl)isoxazol-5-yl)-3-methylbutanoyl)-4-hydroxy-N-((S)-1-(4-(4-methylthiazol-5-

yl)phenyl)ethyl)pyrrolidine-2-carboxamide formate ( $266 \mathrm{mg}, 0.23 \mathrm{mmol}, 39 \%$ yield) was obtained as a white solid. ${ }^{1}$ H NMR (400MHz, DMSO- $\left.d_{6}\right) \delta: 9.02-8.78(\mathrm{~m}, 1 \mathrm{H}), 8.30(\mathrm{br} \mathrm{d}, J=7.2 \mathrm{~Hz}, 1 \mathrm{H}), 8.05(\mathrm{~s}, 1 \mathrm{H}), 7.81$ (br d, $J=7.6 \mathrm{~Hz}$, 1H), 7.67 (br d, $J=5.6 \mathrm{~Hz}, 1 \mathrm{H}), 7.45-7.25(\mathrm{~m}, 5 \mathrm{H}), 7.12(\mathrm{br} \mathrm{t}, J=7.6 \mathrm{~Hz}, 1 \mathrm{H}), 6.83-6.67(\mathrm{~m}, 2 \mathrm{H}), 6.55$ - $6.50(\mathrm{~m}$, $1 \mathrm{H}), 6.18-6.10(\mathrm{~m}, 2 \mathrm{H}), 6.09-5.93(\mathrm{~m}, 2 \mathrm{H}), 5.25-5.15(\mathrm{~m}, 1 \mathrm{H}), 5.13-4.97(\mathrm{~m}, 1 \mathrm{H}), 4.96-4.86(\mathrm{~m}, 1 \mathrm{H}), 4.50(\mathrm{br}$ $\mathrm{s}, 2 \mathrm{H}), 4.40-4.25(\mathrm{~m}, 3 \mathrm{H}), 3.77-3.45(\mathrm{~m}, 4 \mathrm{H}), 3.27-3.12(\mathrm{~m}, 6 \mathrm{H}), 3.06-2.72(\mathrm{~m}, 8 \mathrm{H}), 2.46(\mathrm{~s}, 3 \mathrm{H}), 2.38-2.05(\mathrm{~m}$, $8 \mathrm{H}), 2.03-1.62(\mathrm{~m}, 11 \mathrm{H}), 1.48-1.34(\mathrm{~m}, 3 \mathrm{H}), 1.22(\mathrm{br} \mathrm{d}, J=12.0 \mathrm{~Hz}, 2 \mathrm{H}), 1.02-0.90(\mathrm{~m}, 3 \mathrm{H}), 0.88-0.73(\mathrm{~m}, 3 \mathrm{H})$. (ESI) $\mathrm{m} / \mathrm{z}$ calculated for $\mathrm{C}_{61} \mathrm{H}_{76} \mathrm{~N}_{12} \mathrm{O}_{7} \mathrm{~S}+\mathrm{H}^{+}[\mathrm{M}+\mathrm{H}]^{+}$: 1121.6. Found: 1121.7. 
tert-Butyl (2S,4S)-4-hydroxy-2-[[(1S)-1-[4-(4-methylthiazol-5-yl)phenyl] ethyl]carbamoyl]pyrrolidine-1carboxylate (25)

A mixture of (1S)-1-[4-(4-methylthiazol-5-yl)phenyl]ethanamine (2.4 g, $9.42 \mathrm{mmol}, 1$ eq, hydrochloride), (2S,4S)1-tert-butoxycarbonyl-4-hydroxy-pyrrolidine-2-carboxylic acid $(2.18 \mathrm{~g}, 9.42 \mathrm{mmol}, 1 \mathrm{eq})$, O-(7-azabenzotriazol-1yl)- $N, N, N^{\prime}, N^{\prime}$-tetramethyluronium hexafluorophosphate $(4.30 \mathrm{~g}, 11.30 \mathrm{mmol}, 1.2 \mathrm{eq})$, and triethylamine $(2.86 \mathrm{~g}$, $28.26 \mathrm{mmol}, 3.93 \mathrm{~mL}, 3 e q)$ in $N, N$-dimethylformamide $(40 \mathrm{~mL})$ was degassed and purged with nitrogen 3 times. The mixture was then stirred at $25^{\circ} \mathrm{C}$ for $1 \mathrm{~h}$ under nitrogen. The reaction mixture was diluted with water $(200 \mathrm{~mL})$ and extracted with ethyl acetate $(200 \mathrm{~mL} \times 2)$. The combined organic phase was washed with brine $(50 \mathrm{~mL})$, dried over anhydrous sodium sulfate, filtered, and concentrated in vacuum. The residue was purified by preparative HPLC (column: Phenomenex luna C18 250*50 mm*10 um; mobile phase: [water (0.225\% FA) - ACN]; B\%: 25\% - 55\%). tert-Butyl (2S,4S)-4-hydroxy-2-[[(1S)-1-[4-(4-methylthiazol-5-yl)phenyl] ethyl]carbamoyl]pyrrolidine-1carboxylate (3.5 g, $8.11 \mathrm{mmol}, 86 \%$ yield) was obtained as a white solid.

(2S,4S)-1-((2R)-2-(3-(4-((4-((1r,3r)-3-((4-(3-(3-amino-6-(2-hydroxyphenyl)pyridazin-4-yl)-3,8diazabicyclo[3.2.1 ]octan-8-yl)pyridin-2-yl)oxy)cyclobutoxy)piperidin-1-yl)methyl)piperidin-1-yl)isoxazol-5-yl)-3methylbutanoyl)-4-hydroxy-N-((S)-1-(4-(4-methylthiazol-5-yl)phenyl)ethyl)pyrrolidine-2-carboxamide (A857) (2S,4S)-1-((2R)-2-(3-(4-((4-((1r,3r)-3-((4-(3-(3-amino-6-(2-hydroxyphenyl)pyridazin-4-yl)-3,8-

diazabicyclo[3.2.1]octan-8-yl)pyridin-2-yl)oxy)cyclobutoxy)piperidin-1-yl)methyl)piperidin-1-yl)isoxazol-5-yl)-3methylbutanoyl)-4-hydroxy-N-((S)-1-(4-(4-methylthiazol-5-yl)phenyl)ethyl)pyrrolidine-2-carboxamide was prepared from tert-butyl (2S,4S)-4-hydroxy-2-[[(1S)-1-[4-(4-methylthiazol-5-yl)phenyl] ethyl]carbamoyl]pyrrolidine-1-carboxylate (25) according to the Schemes 6 and 7 using procedures described for the preparation of A947 from intermediate 20. ${ }^{1} \mathbf{H}$ NMR (400 MHz, DMSO- $\left.d_{6}\right) \delta: 9.02-8.99(\mathrm{~m}, 1 \mathrm{H}), 8.39(\mathrm{~d}, J=7.6$ $\mathrm{Hz}, 1 \mathrm{H}), 8.14(\mathrm{~s}, 1 \mathrm{H}), 8.03-7.89(\mathrm{~m}, 1 \mathrm{H}), 7.84-7.73(\mathrm{~m}, 1 \mathrm{H}), 7.52-7.36(\mathrm{~m}, 5 \mathrm{H}), 7.23(\mathrm{t}, J=7.6 \mathrm{~Hz}, 1 \mathrm{H}), 6.95-$ $6.83(\mathrm{~m}, 2 \mathrm{H}), 6.52-6.49(\mathrm{~m}, 1 \mathrm{H}), 6.21-6.12(\mathrm{~m}, 2 \mathrm{H}), 5.99(\mathrm{~s}, 2 \mathrm{H}), 5.37-5.18(\mathrm{~m}, 2 \mathrm{H}), 4.94$ (quin, $J=7.2 \mathrm{~Hz}, 1 \mathrm{H})$, 4.50 (br s, 2H), $4.38-4.24$ (m, 2H), 4.20 (br d, $J=5.6 \mathrm{~Hz}, 1 \mathrm{H}), 3.75-3.59$ (m, 4H), $3.59-3.50$ (m, 2H), $3.29-3.12$ (m, 4H), $3.08-2.72(\mathrm{~m}, 8 \mathrm{H}), 2.46(\mathrm{~s}, 3 \mathrm{H}), 2.40-2.10(\mathrm{~m}, 9 \mathrm{H}), 2.02-1.61(\mathrm{~m}, 10 \mathrm{H}), 1.48-1.34(\mathrm{~m}, 3 \mathrm{H}), 1.31-1.10$ $(\mathrm{m}, 2 \mathrm{H}), 1.01-0.91(\mathrm{~m}, 3 \mathrm{H}), 0.89-0.74(\mathrm{~m}, 3 \mathrm{H})$. (ESI) $\mathrm{m} / \mathrm{z}$ calculated for $\mathrm{C}_{61} \mathrm{H}_{76} \mathrm{~N}_{12} \mathrm{O}_{7} \mathrm{~S}+\mathrm{H}^{+}[\mathrm{M}+\mathrm{H}]^{+}: 1121.6$. Found: 1121.8 .

(2S,4R)-1-((2R)-2-(3-(4-((4-((1r,3r)-3-((4-(3-(3-amino-6-phenylpyridazin-4-yl)-3,8-diazabicyclo[3.2.1]octan-8yl)pyridin-2-yl)oxy)cyclobutoxy)piperidin-1-yl)methyl)piperidin-1-yl)isoxazol-5-yl)-3-methylbutanoyl)-4-hydroxyN-((S)-1-(4-(4-methylthiazol-5-yl)phenyl)ethyl)pyrrolidine-2-carboxamide (A858)

(2S,4R)-1-((2R)-2-(3-(4-((4-((1r,3r)-3-((4-(3-(3-amino-6-phenylpyridazin-4-yl)-3,8-diazabicyclo[3.2.1]octan-8yl)pyridin-2-yl)oxy)cyclobutoxy)piperidin-1-yl)methyl)piperidin-1-yl)isoxazol-5-yl)-3-methylbutanoyl)-4-hydroxyN-((S)-1-(4-(4-methylthiazol-5-yl)phenyl)ethyl)pyrrolidine-2-carboxamide (A858) was prepared from tert-butyl 4((1r,3r)-3-((4-(3-(3-amino-6-chloropyridazin-4-yl)-3,8-diazabicyclo[3.2.1] octan-8-yl)pyridin-2-

yl)oxy)cyclobutoxy)piperidine-1-carboxylate (9) according to the Scheme 8 using procedures described for the preparation of A947. ${ }^{1} \mathbf{H}$ NMR (400 MHz, DMSO- $\left.d_{6}\right) \delta: 8.97(\mathrm{~s}, 1 \mathrm{H}), 8.37(\mathrm{~d}, J=7.6 \mathrm{~Hz}, 1 \mathrm{H}), 8.12(\mathrm{~s}, 1 \mathrm{H}), 7.95$ $(\mathrm{d}, J=7.2 \mathrm{~Hz}, 1 \mathrm{H}), 7.74(\mathrm{~d}, J=6.0 \mathrm{~Hz}, 1 \mathrm{H}), 7.46-7.39(\mathrm{~m}, 3 \mathrm{H}), 7.39-7.31(\mathrm{~m}, 3 \mathrm{H}), 7.24(\mathrm{~s}, 1 \mathrm{H}), 6.61-6.41(\mathrm{~m}$, $2 \mathrm{H}), 6.21-5.96(\mathrm{~m}, 2 \mathrm{H}), 5.71(\mathrm{~s}, 2 \mathrm{H}), 5.27-5.13(\mathrm{~m}, 1 \mathrm{H}), 5.09(\mathrm{br} \mathrm{d}, J=2.0 \mathrm{~Hz}, 1 \mathrm{H}), 4.89(\mathrm{brt}, J=7.2 \mathrm{~Hz}, 1 \mathrm{H})$, 4.46 (br s, 2H), 4.34 (t, $J=8.0 \mathrm{~Hz}, 1 \mathrm{H}), 4.30-4.22$ (m, 2H), 3.69 (br dd, $J=4.4,10.4 \mathrm{~Hz}, 1 \mathrm{H}), 3.61$ (br d, $J=12.0$ $\mathrm{Hz}, 2 \mathrm{H}), 3.57-3.52(\mathrm{~m}, 1 \mathrm{H}), 3.41$ (br d, $J=2.8 \mathrm{~Hz}, 4 \mathrm{H}), 3.19$ (br d, $J=11.2 \mathrm{~Hz}, 3 \mathrm{H}), 2.92$ (br d, $J=11.2 \mathrm{~Hz}, 4 \mathrm{H})$, $2.78-2.69(\mathrm{~m}, 2 \mathrm{H}), 2.62-2.54(\mathrm{~m}, 1 \mathrm{H}), 2.44(\mathrm{~s}, 4 \mathrm{H}), 2.33-2.25(\mathrm{~m}, 4 \mathrm{H}), 2.22-2.10(\mathrm{~m}, 3 \mathrm{H}), 2.00(\mathrm{br} \mathrm{s}, 1 \mathrm{H}), 1.96$ - $1.90(\mathrm{~m}, 2 \mathrm{H}), 1.89-1.82(\mathrm{~m}, 2 \mathrm{H}), 1.81-1.66(\mathrm{~m}, 4 \mathrm{H}), 1.64-1.50(\mathrm{~m}, 2 \mathrm{H}), 1.47-1.30(\mathrm{~m}, 3 \mathrm{H}), 1.26$ - $1.07(\mathrm{~m}$, $2 \mathrm{H}), 1.01-0.86(\mathrm{~m}, 3 \mathrm{H}), 0.77(\mathrm{~d}, J=6.8 \mathrm{~Hz}, 3 \mathrm{H})$. (ESI) $\mathrm{m} / \mathrm{z}$ calculated for $\mathrm{C}_{61} \mathrm{H}_{76} \mathrm{~N}_{12} \mathrm{O}_{6} \mathrm{~S}+\mathrm{H}^{+}[\mathrm{M}+\mathrm{H}]^{+}: 1105.6$. Found: 1105.6.

(2S,4R)-4-hydroxy-1-[(2R)-2-(3-methoxy-1,2-oxazol-5-yl)-3-methylbutanoyl]-N-[[4-(4-methyl-1,3-thiazol-5yl)phenyl]methyl]pyrrolidine-2-carboxamide (A2702)

Into a 50-mL round-bottom flask was placed a solution of (2S,4R)-4-hydroxy-N-[[4-(4-methyl-1,3-thiazol-5yl)phenyl]methyl]pyrrolidine-2-carboxamide hydrochloride 31 [prepared as described for the intermediate $\mathbf{2 0}$ above] (200.0 mg, $0.57 \mathrm{mmol}, 1 \mathrm{eq})$ in N,N-dimethylformamide (10 mL). N,N,N',N'-Tetramethyl-O-(7-azabenzotriazol-1yl)uronium hexafluorophospate ( $450 \mathrm{mg}, 1.18 \mathrm{mmol}, 1.2 \mathrm{eq}$ ), N,N-diisopropylethylamine ( $520 \mathrm{mg}, 4.02 \mathrm{mmol}, 4$ eq), 2-(3-methoxy-1,2-oxazol-5-yl)-3-methylbutanoic acid² (317 mg, $1.59 \mathrm{mmol}, 1 \mathrm{eq})$ were added. The resulting 
solution was stirred for 2 hours at room temperature. The reaction was then quenched by the addition of $20 \mathrm{~mL}$ of water and extracted with ethyl acetate $(100 \mathrm{~mL} \times 2)$. The organic layers were combined and concentrated under reduced pressure. The residue was initially purified on a silica gel column eluting with ethyl acetate. The product

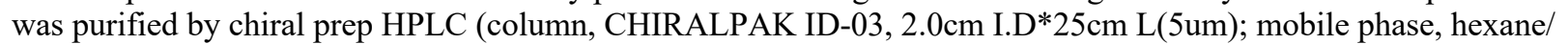
IPA (hold 50\% IPA; 20 min). (2S,4R)-4-Hydroxy-1-[(2R)-2-(3-methoxy-1,2-oxazol-5-yl)-3-methylbutanoyl]-N-[[4(4-methyl-1,3-thiazol-5-yl)phenyl]methyl]pyrrolidine-2-carboxamide (20.4 $\mathrm{mg}, 0.04 \mathrm{mmol}, 8 \%$ yield) was produced as a white solid. ${ }^{1} \mathbf{H}$ NMR $\left(400 \mathrm{MHz}\right.$, methanol- $\left.d_{4}\right): \delta 8.90(\mathrm{~s}, 1 \mathrm{H}), 7.51-7.41(\mathrm{~m}, 4 \mathrm{H}), 5.99(\mathrm{~s}, 1 \mathrm{H}), 4.62-4.48(\mathrm{~m}$, 4H), 3.96-3.89 (m, 4H), 3.79-3.73 (m, 2H), $2.50(\mathrm{~d}, \mathrm{~J}=3.8 \mathrm{~Hz}, 3 \mathrm{H}), 2.46-2.29(\mathrm{~m}, 1 \mathrm{H}), 2.31-2.18(\mathrm{~m}, 1 \mathrm{H}), 2.09-$ $2.01(\mathrm{~m}, 1 \mathrm{H}), 1.07(\mathrm{~d}, \mathrm{~J}=6.7 \mathrm{~Hz}, 3 \mathrm{H}), 0.93(\mathrm{~d}, \mathrm{~J}=6.7 \mathrm{~Hz}, 3 \mathrm{H})$. $(\mathrm{ESI}) \mathrm{m} / \mathrm{z}$ calculated for $\mathrm{C}_{25} \mathrm{H}_{30} \mathrm{~N}_{4} \mathrm{O}_{5} \mathrm{~S}+\mathrm{H}^{+}[\mathrm{M}+\mathrm{H}]^{+}$ : 499.2. Found: 499.0 .

1. Crew, A. et al. Tetrahydronaphthalene and tetrahydroisoquinoline derivatives as estrogen receptor degraders and their preparation. PCT Int. Appl. (2018), WO 2018102725.

2. Yimin, Q. et al. Preparation of bifunctional PROTAC compounds and methods for the enhanced degradation of targeted bromodomain-containing proteins. PCT Int. Appl. (2017), WO 2017030814. 
Scheme 1. Synthesis of Intermediate 5
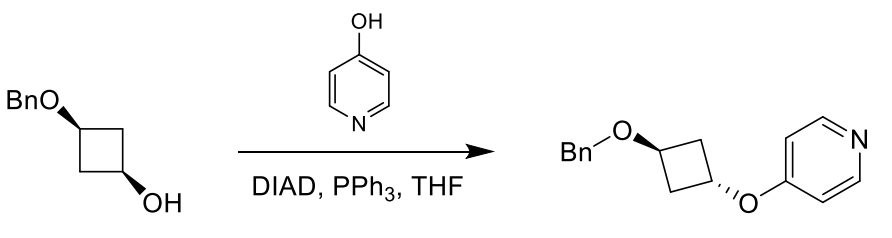

1

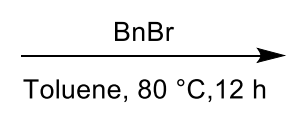

Toluene, $80^{\circ} \mathrm{C}, 12 \mathrm{~h}$

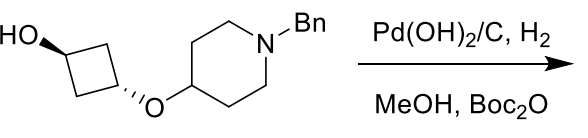

$\stackrel{\mathrm{NaBH}_{4}}{\longrightarrow} \mathrm{Bn}_{3}^{-\mathrm{O}} \underset{\mathrm{MeOH}}{\mathrm{Pd}(\mathrm{OH})_{2} / \mathrm{C}, \mathrm{H}_{2}}$

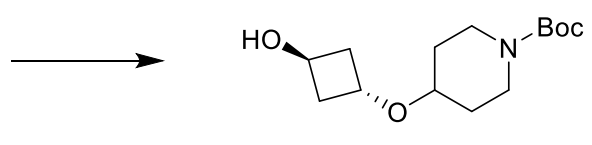


Scheme 2. Synthesis of Intermediate 11
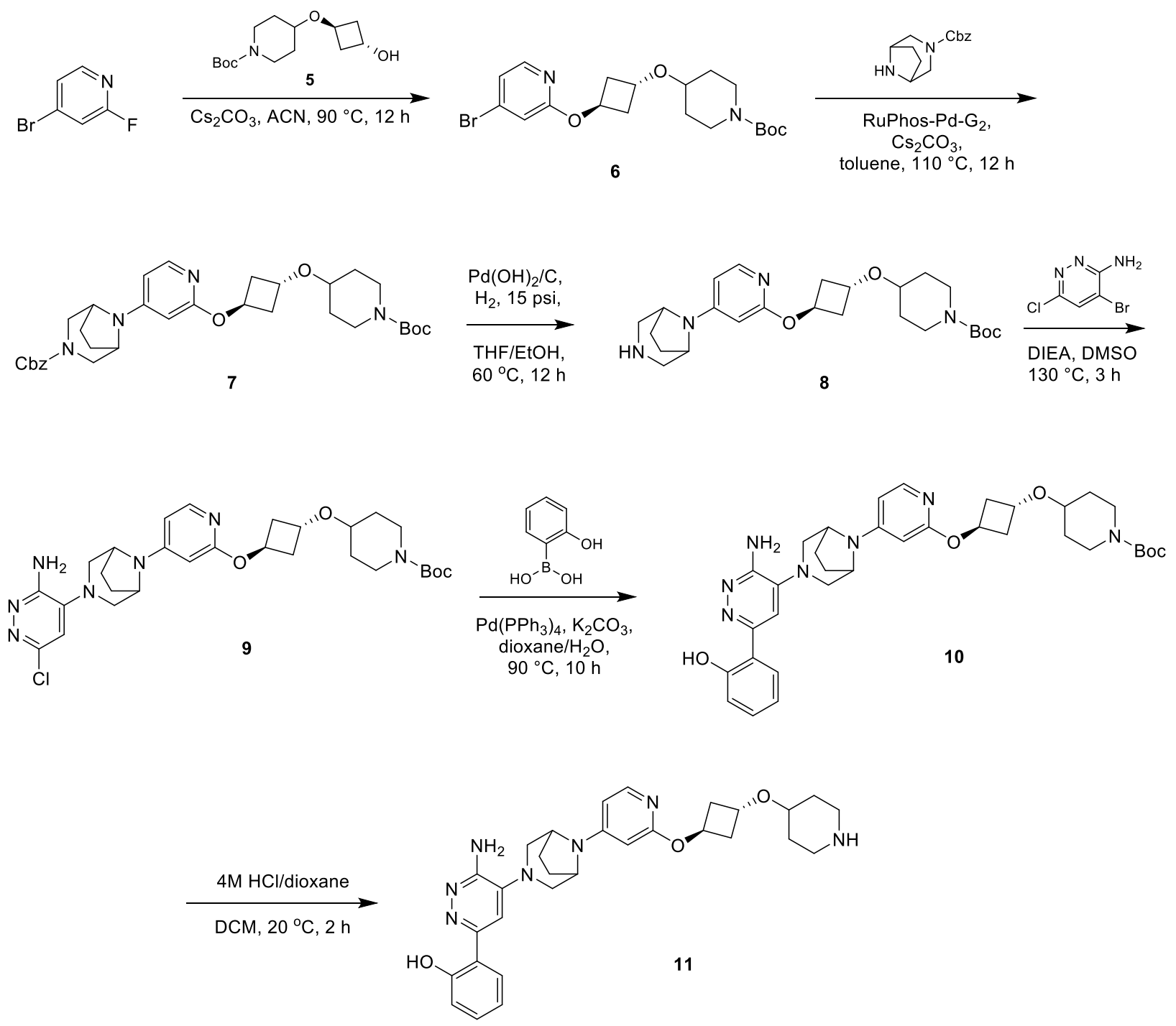
Scheme 3. Synthesis of intermediate 20

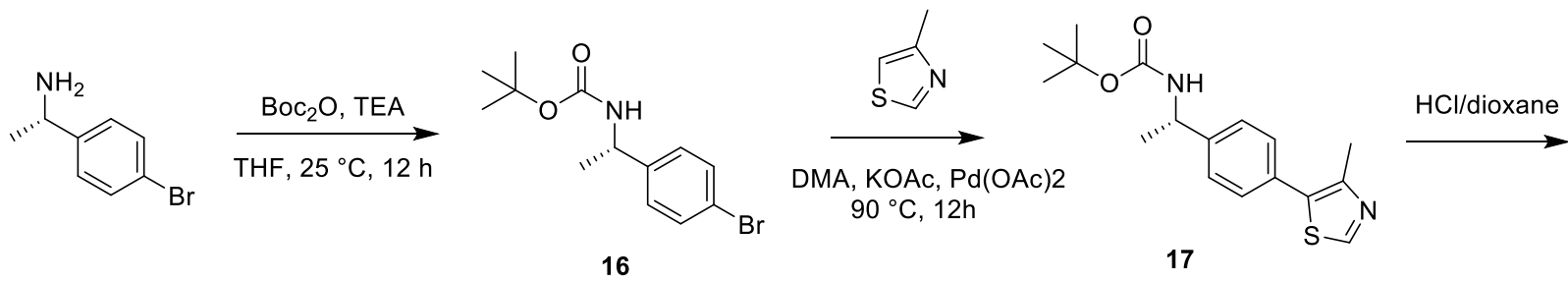

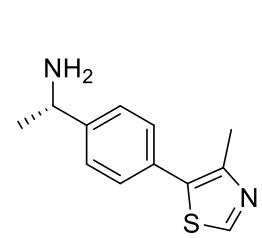

18

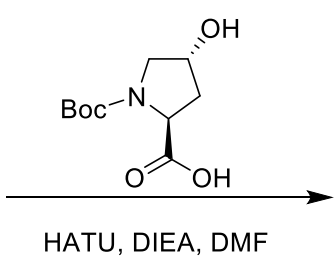

HATU, DIEA, DMF

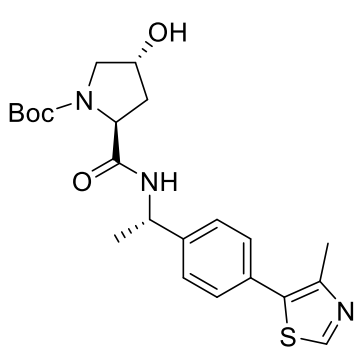

19

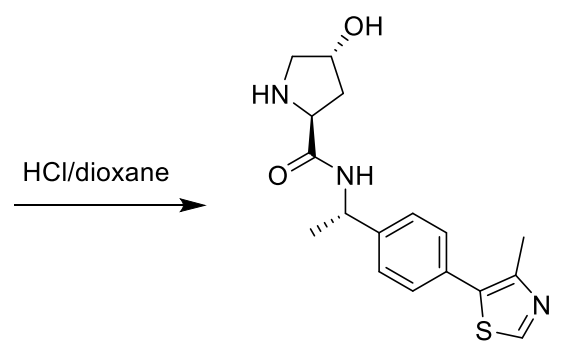

20 
Scheme 4. Synthesis of intermediate 24

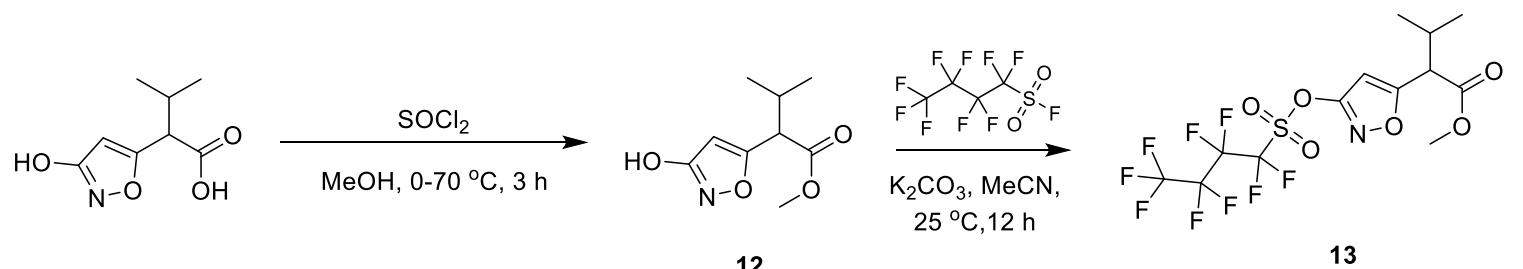

12

13

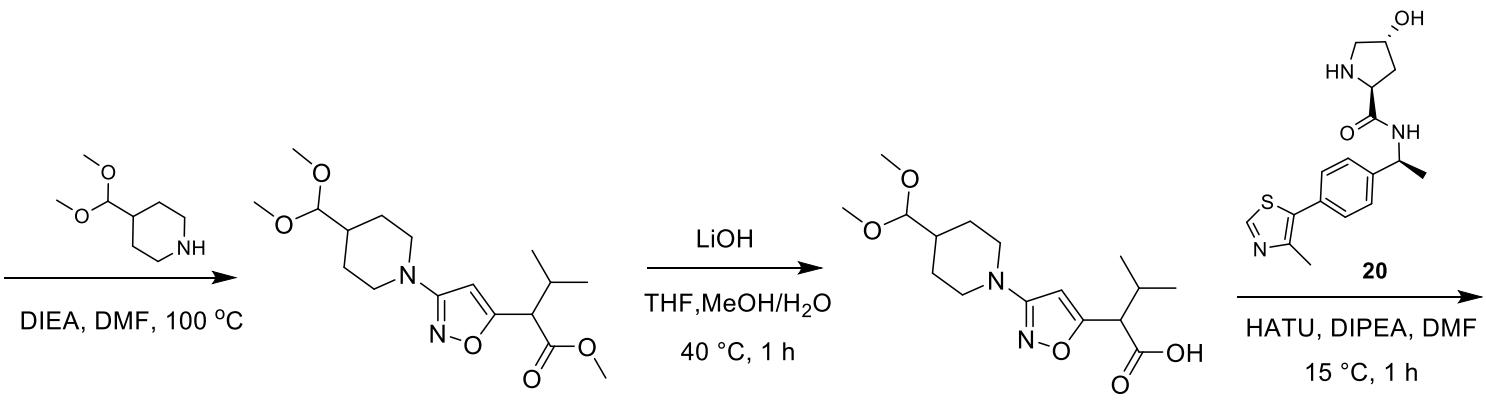

14

15

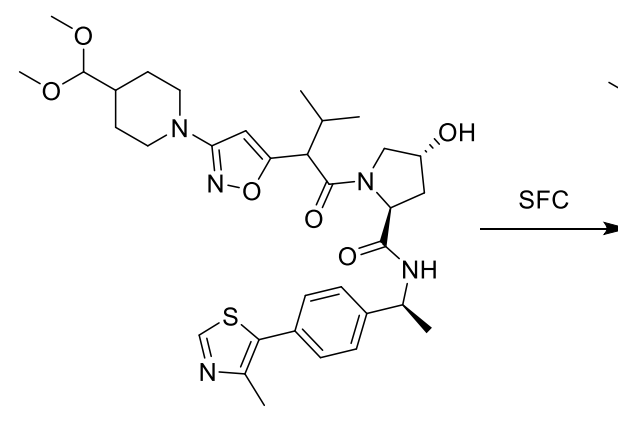

21

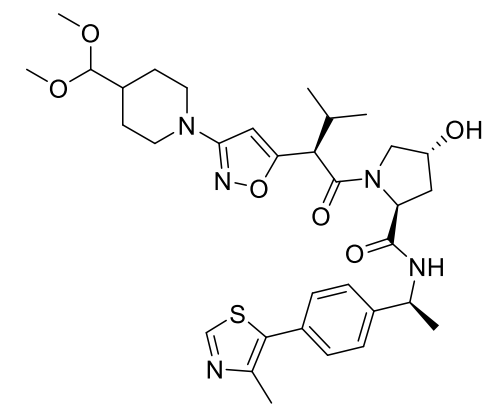

23

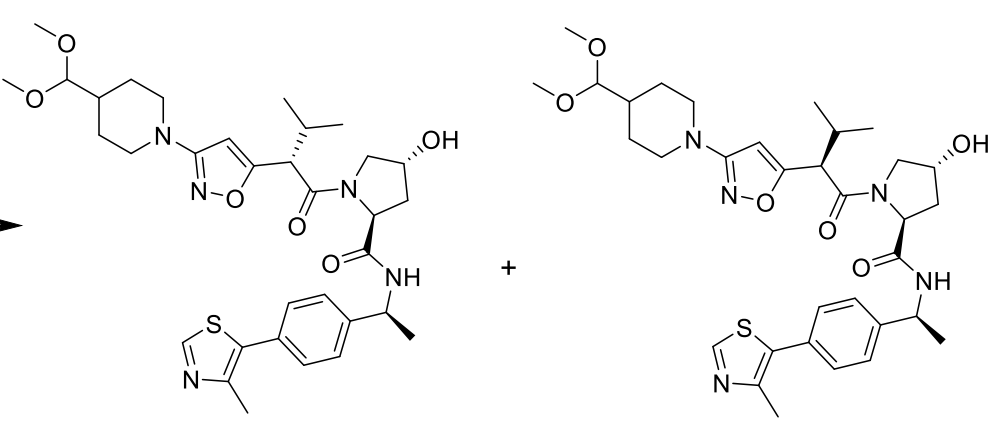

22

23

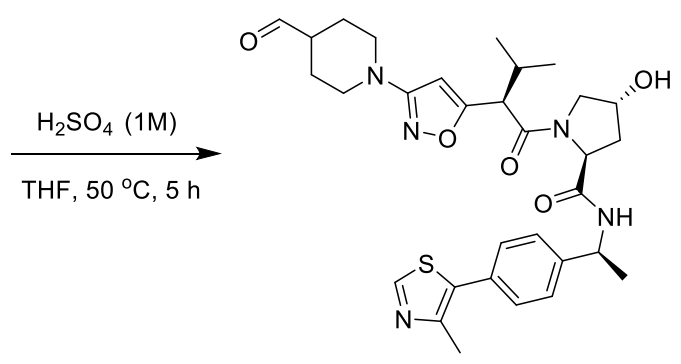

24 
Scheme 5. Synthesis of A947

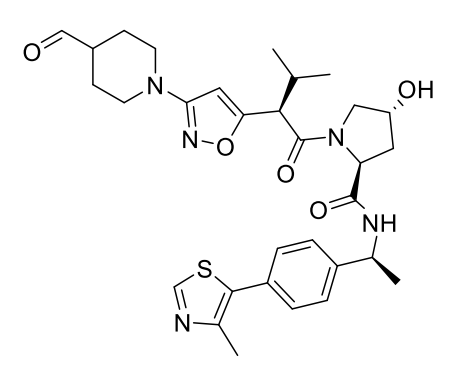

24
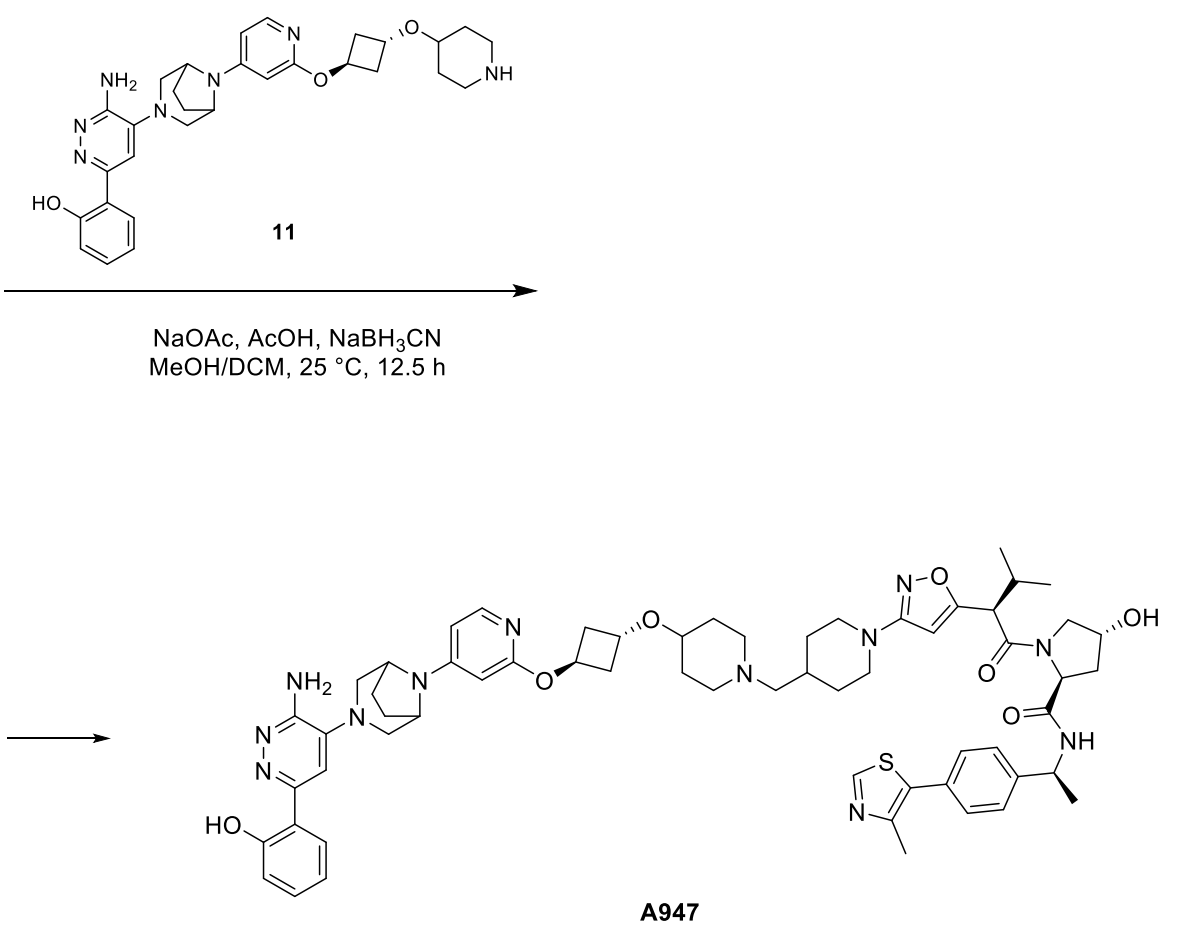
Scheme 6. Synthesis of intermediate 29

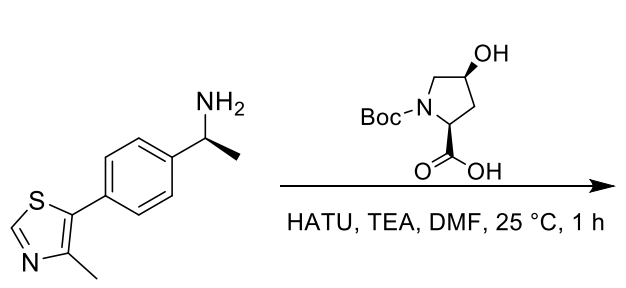

18

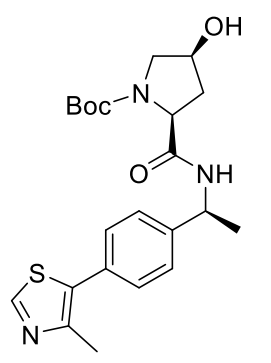

25

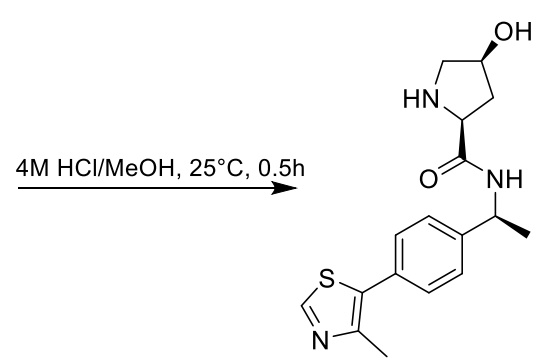

26

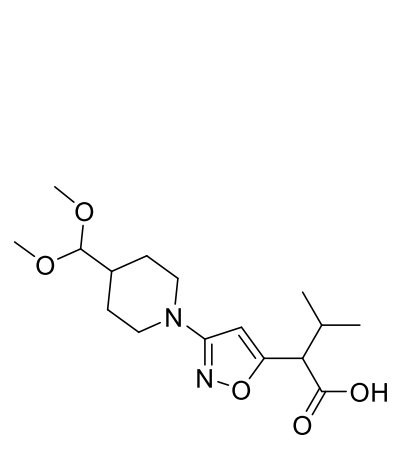

15
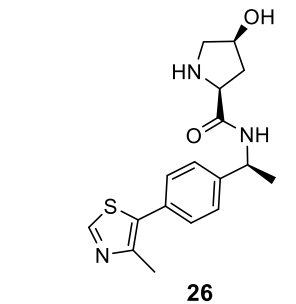

HATU, TEA, DMF $25^{\circ} \mathrm{C}, 0.5 \mathrm{~h}$
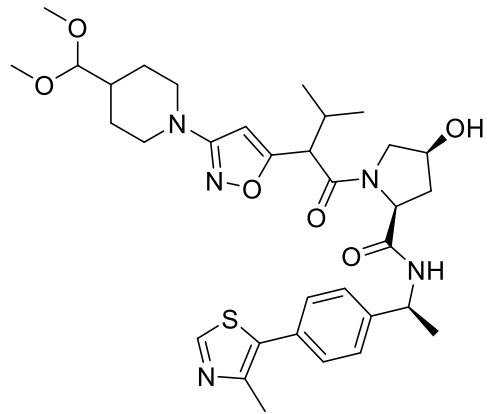

27

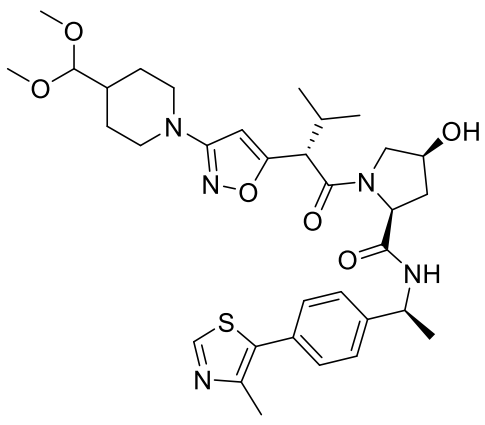

28

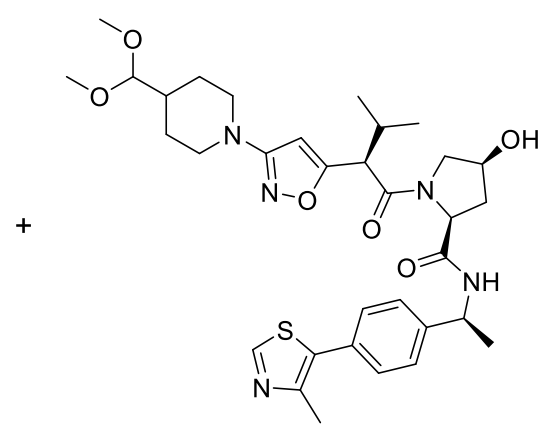

29 
Scheme 7. Synthesis of A857

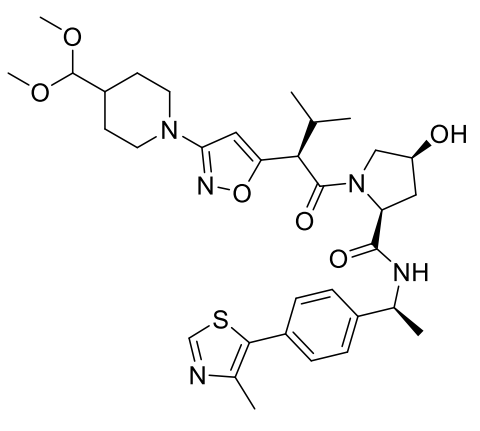

29
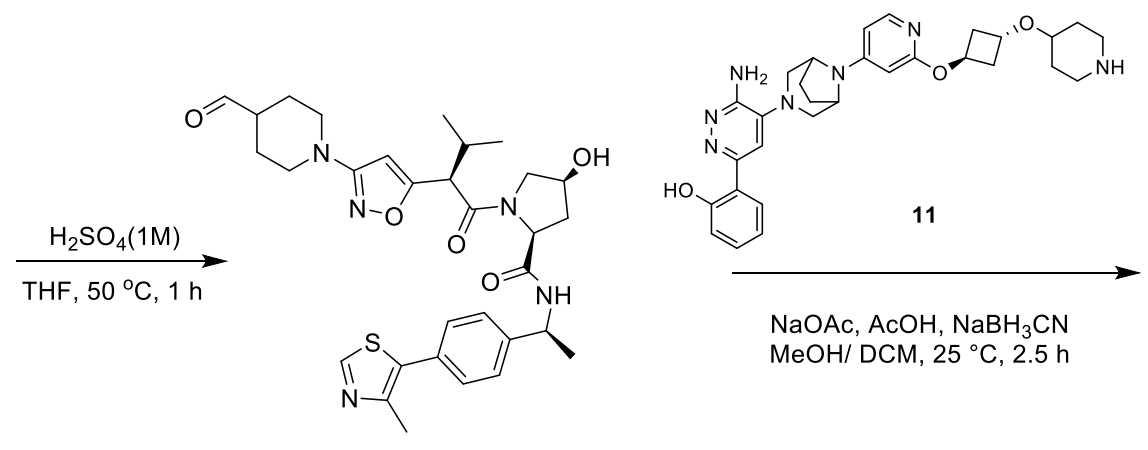

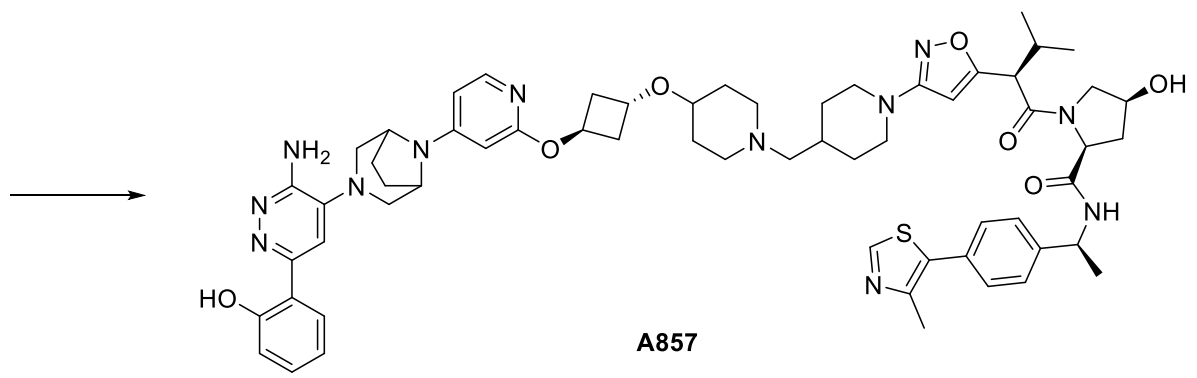


Scheme 8. Synthesis of $\mathbf{A 8 5 8}$
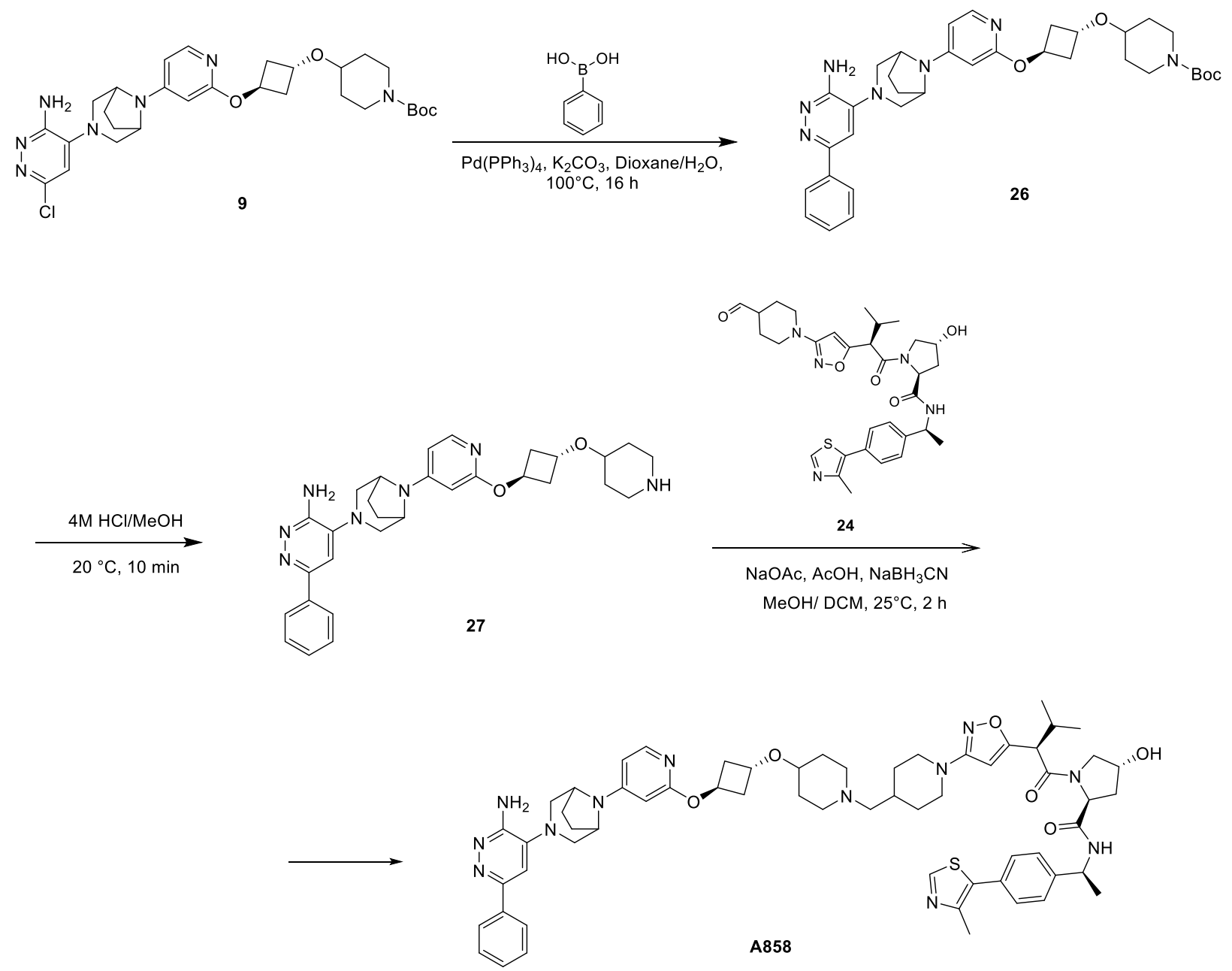
Scheme 9. Synthesis of A2702

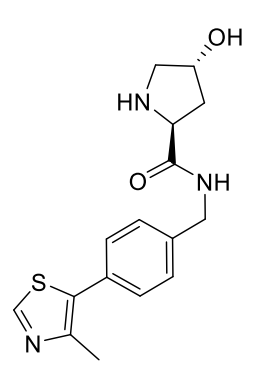

31

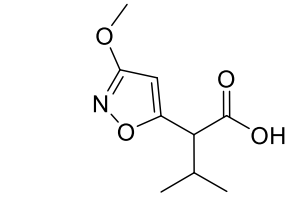

HATU, DIEA, DMF, rt, $2 \mathrm{~h}$

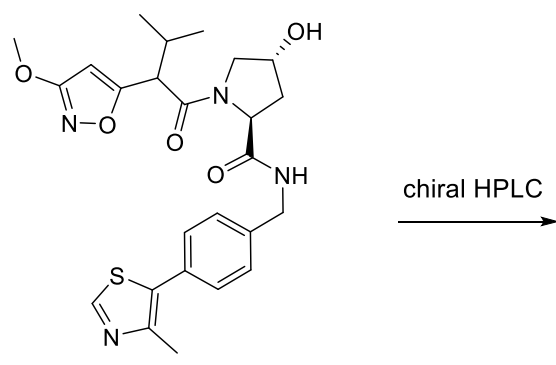

32

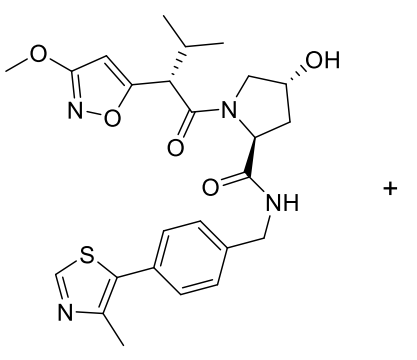

33

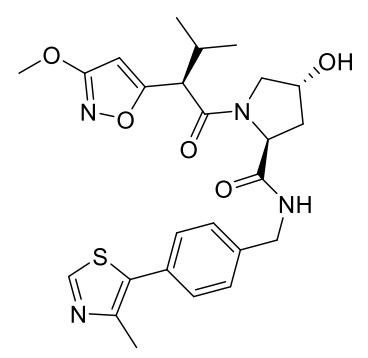

A2702 
Fig. 1.
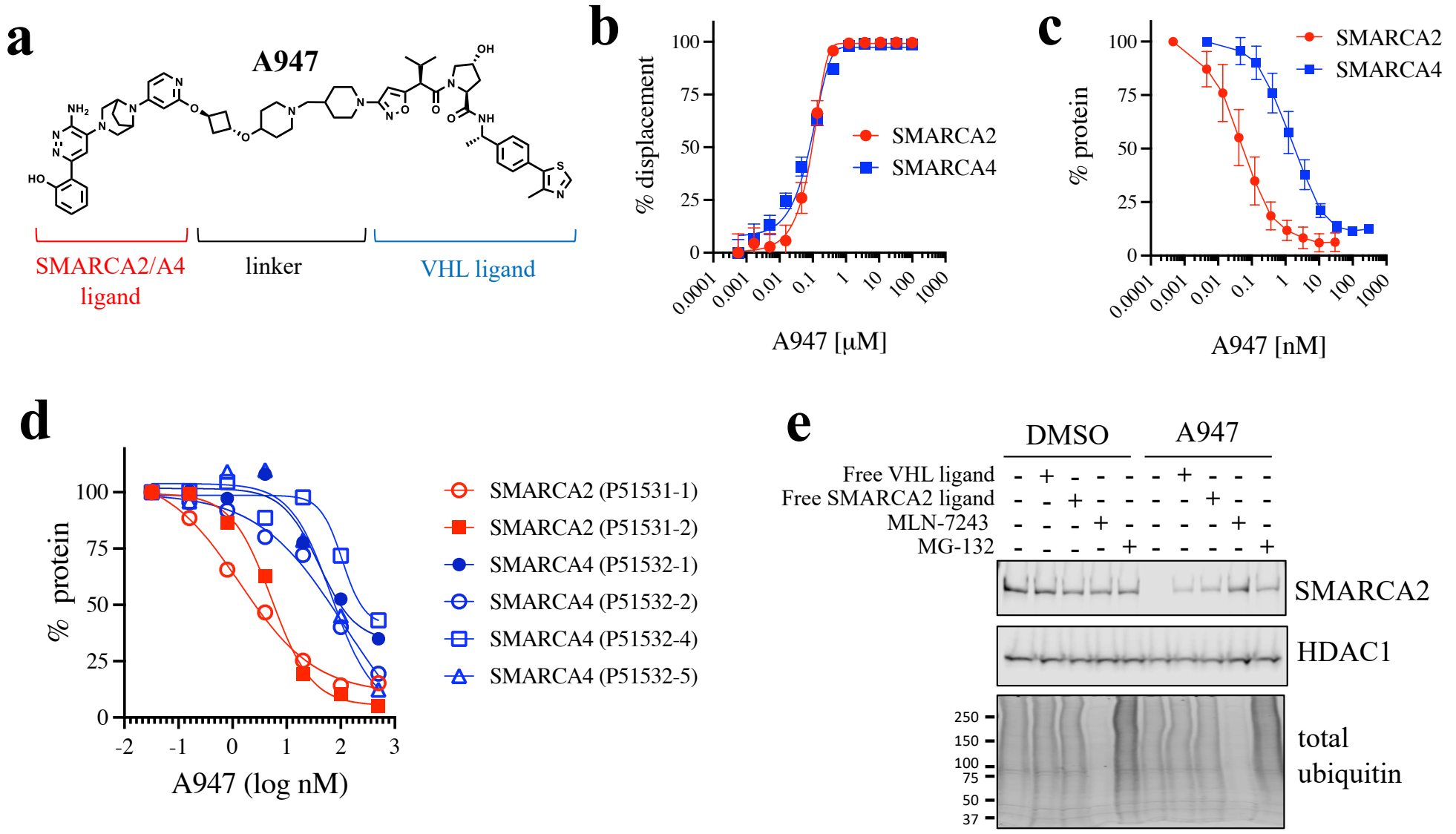

f

TOV112D (human) cells

LA-4 (murine) cells

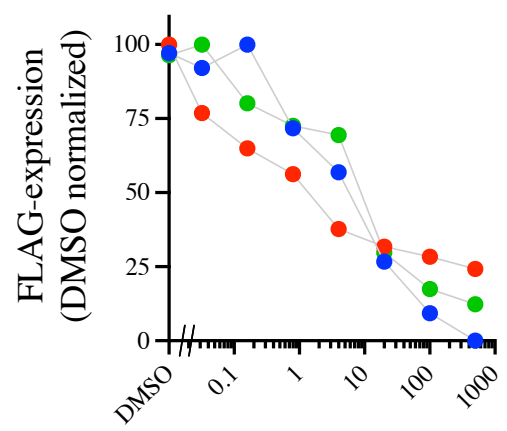

A947 (nM)

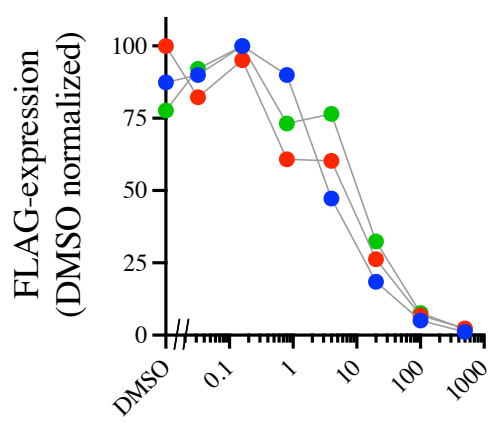

A947 (nM)
- murine SMARCA2-flag

- rat SMARCA2-flag

- human SMARCA2-flag 
Fig. 2

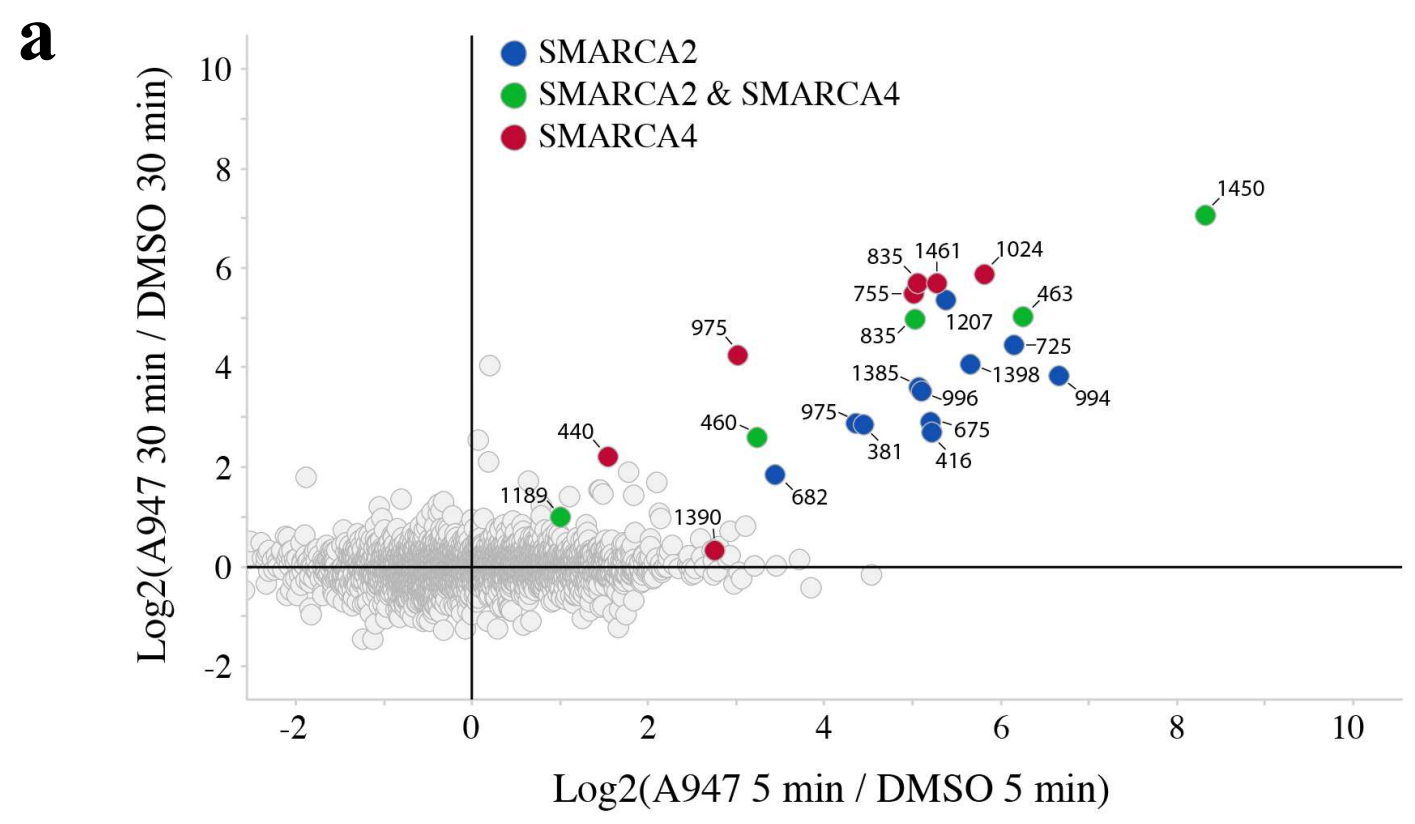

b

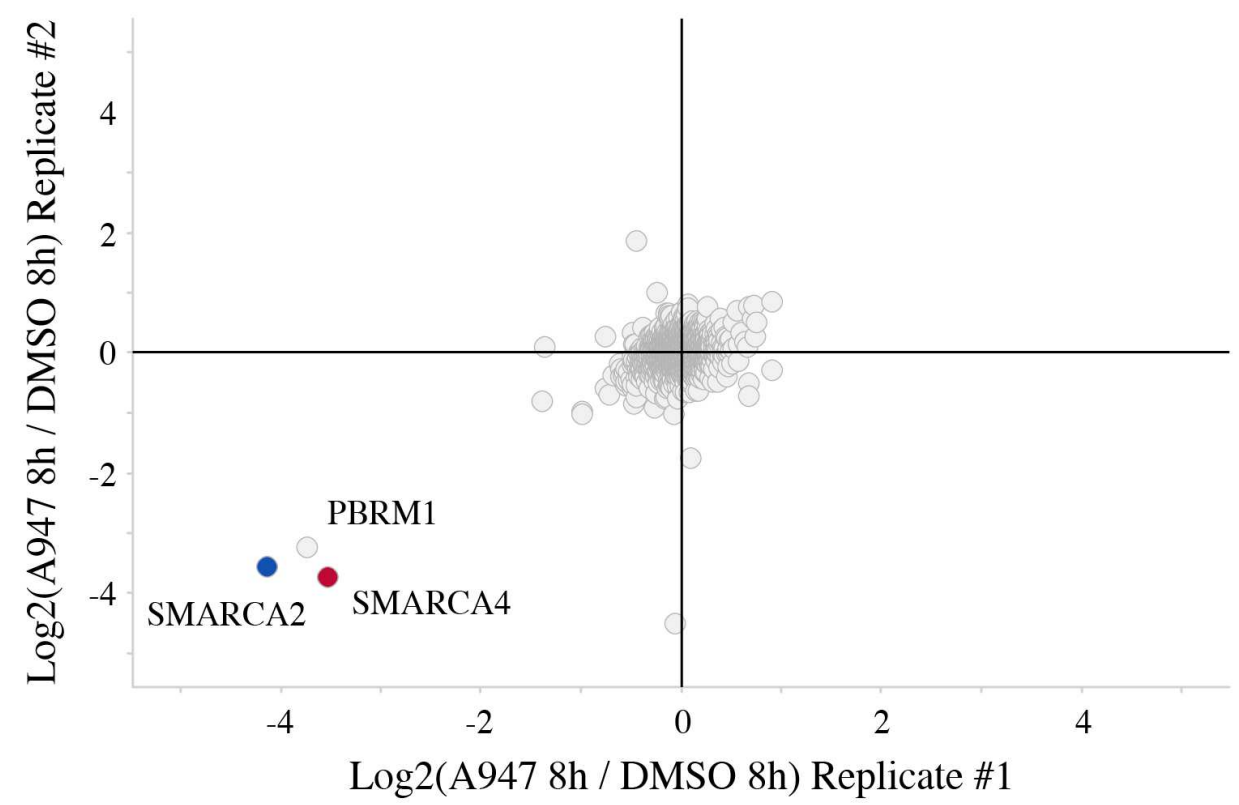


Fig. 3

a

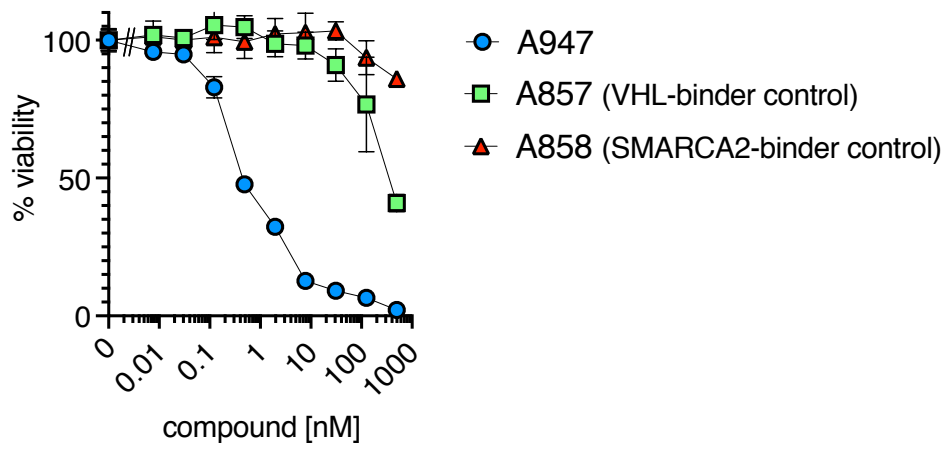

b

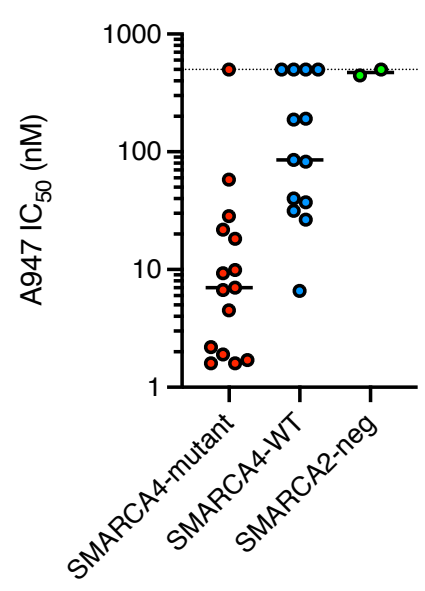

c
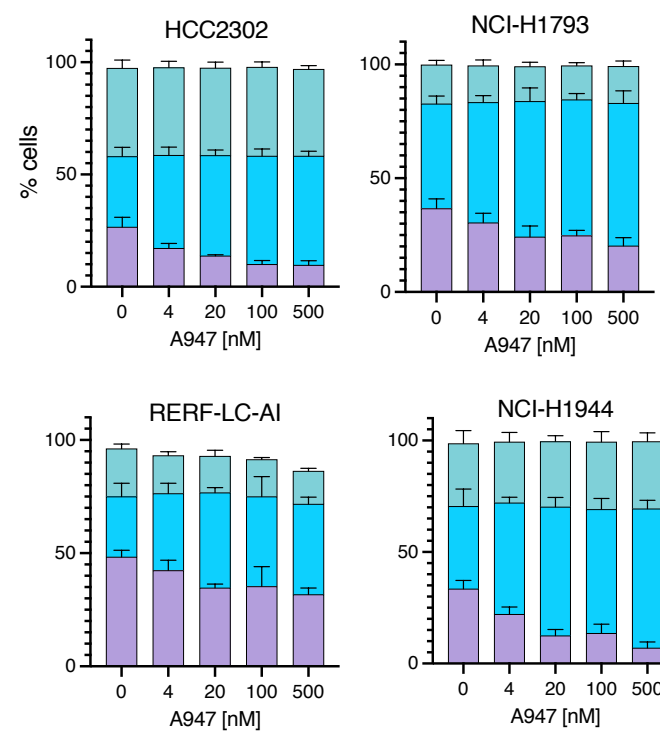

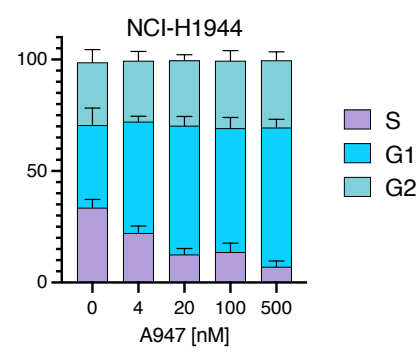

d

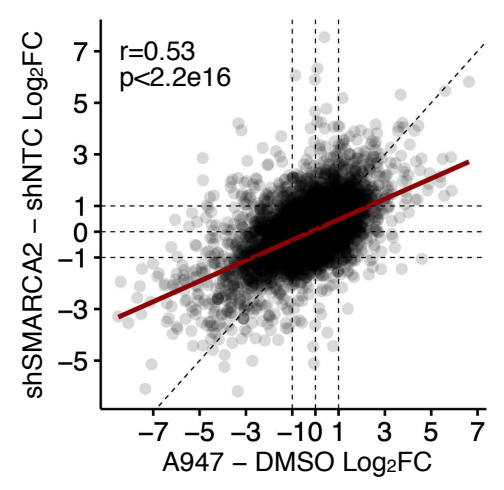


Fig. 4

a
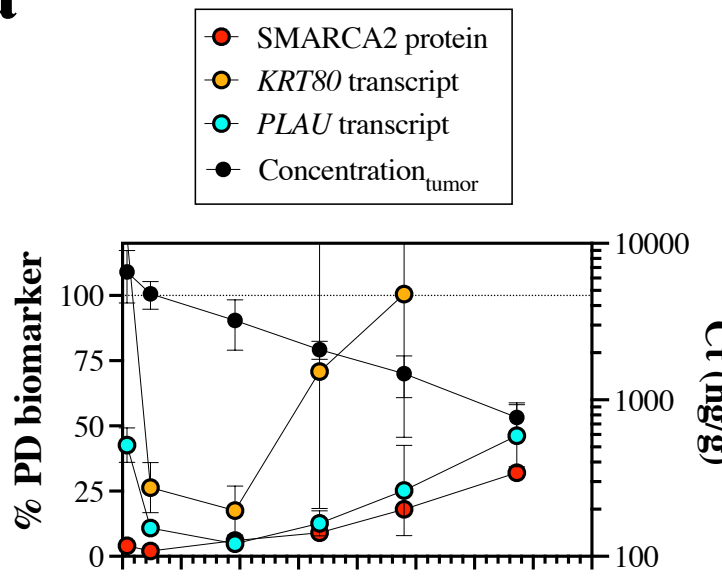

$0 \quad 50 \quad 100 \quad 150200 \quad 250300350400$

time (hr)

c

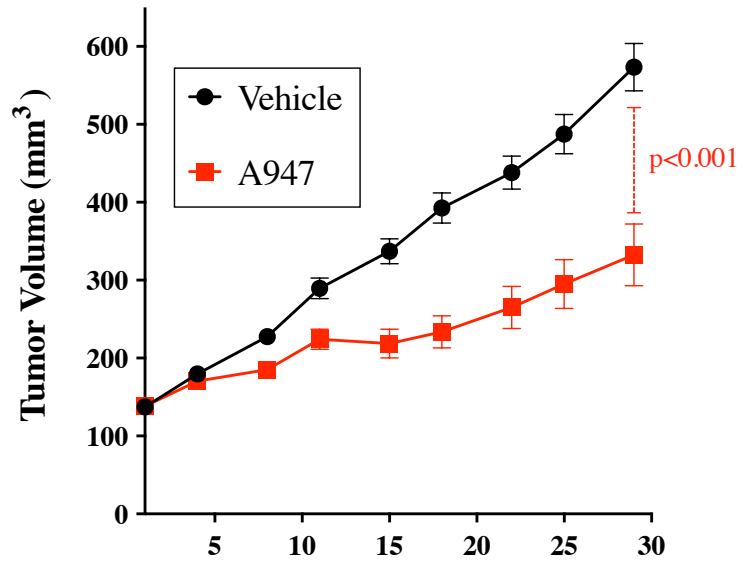

Days of tumor growth

f

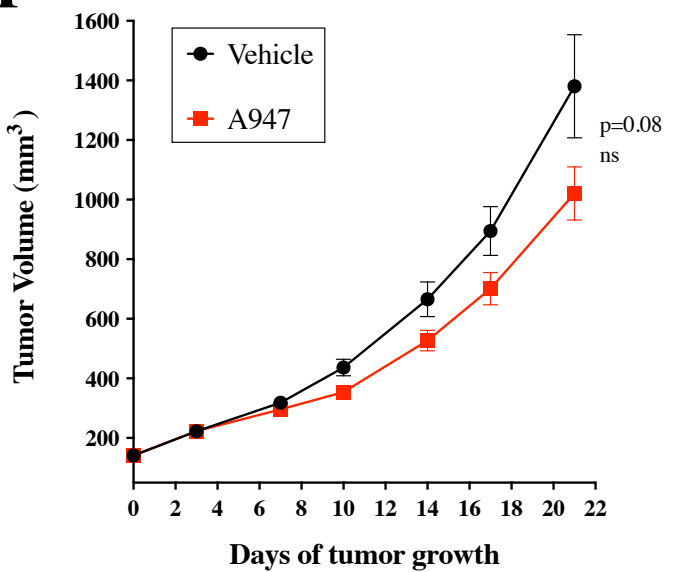

b

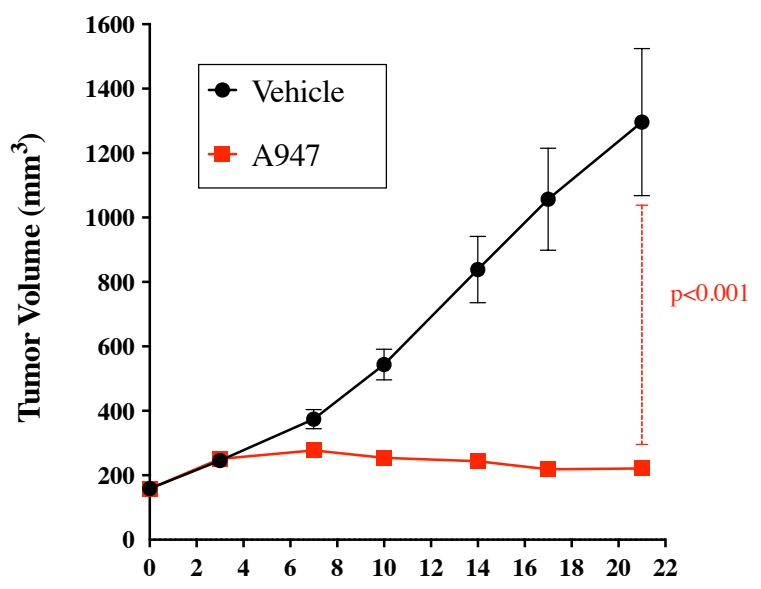

Days of tumor growth

d

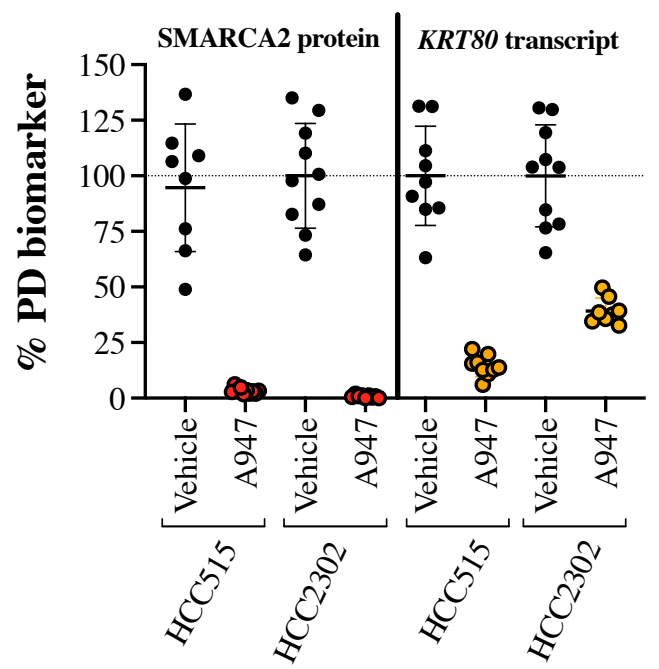

$\delta$

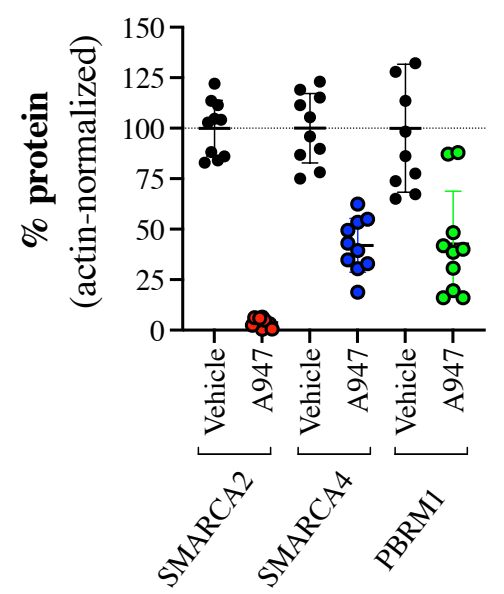


Fig. 5

a

b
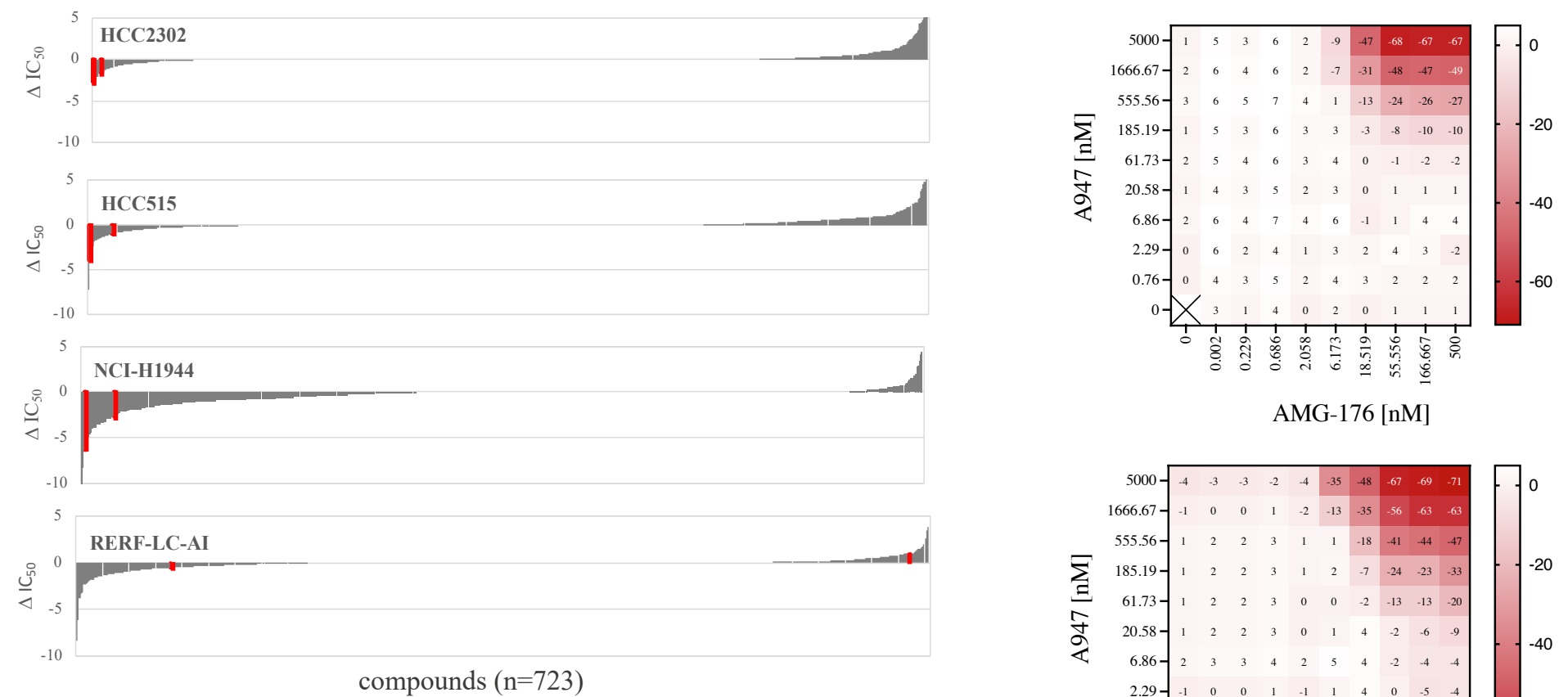

compounds $(\mathrm{n}=723)$

c
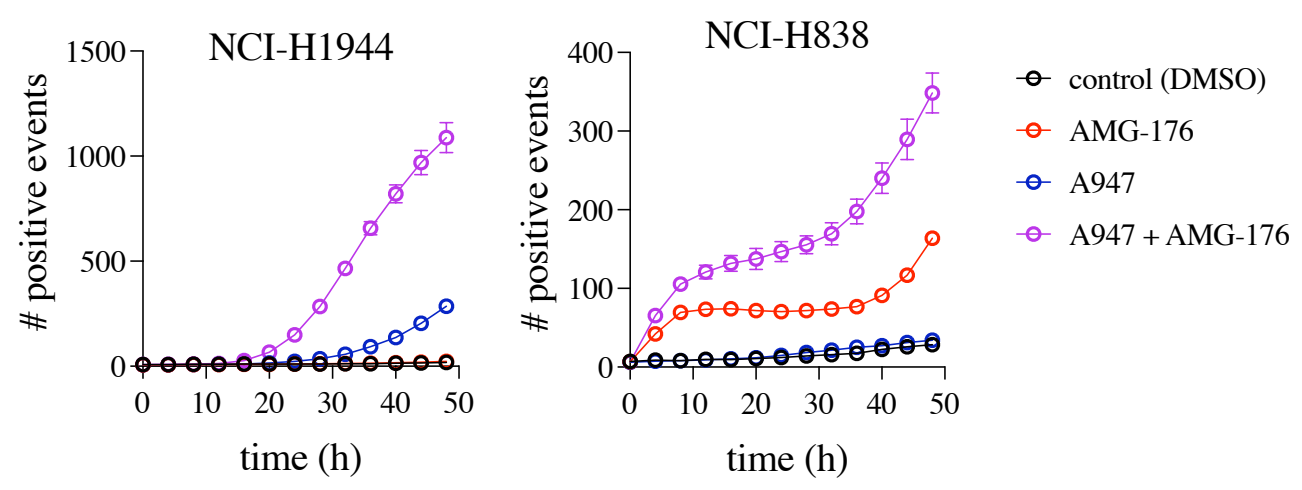
Extended Data Fig. 1.

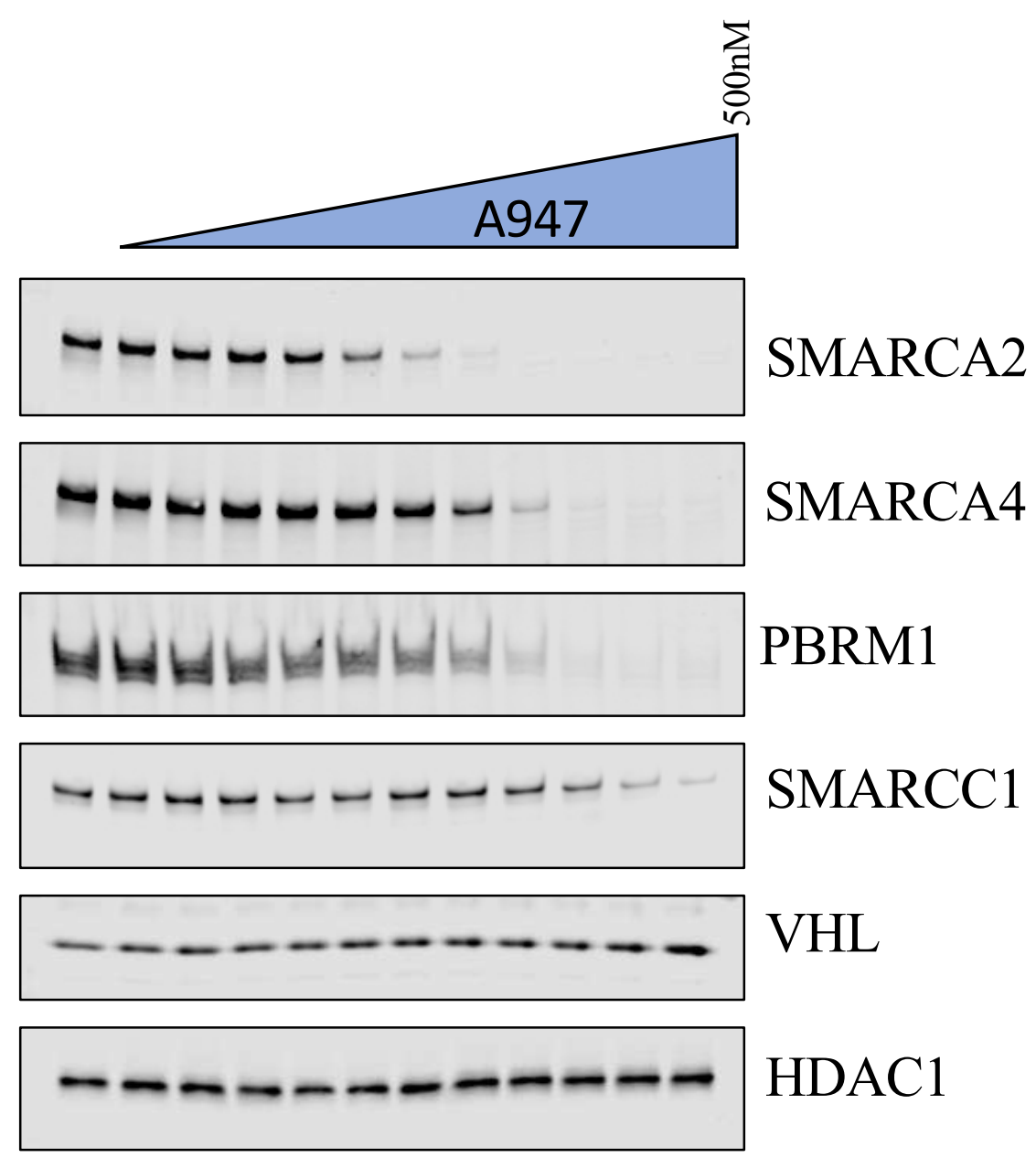




\section{Extended Data Fig. 2.}

$\mathbf{a}$

\begin{tabular}{|c|c|c|c|c|c|c|c|}
\hline Gene & UniProt & UniProt isoform & $\begin{array}{c}\text { NCBI Isoform } \\
\text { definition }\end{array}$ & $\begin{array}{c}\text { Difference from canonical } \\
\text { sequence }\end{array}$ & AA length & $\begin{array}{l}\text { NCBI mRNA } \\
\text { Reference }\end{array}$ & $\begin{array}{c}\text { NCBI reference } \\
\text { sequence }\end{array}$ \\
\hline SMARCA2 & P51531-1 & isoform 1 & isoform a & & 1590 & NM 003070 & NP 003061.3 \\
\hline SMARCA2 & P51531-2 & isoform 2 & isoform b & 1400-1417: Missing & 1572 & NM_139045.3 & NP_620614.2 \\
\hline SMARCA4 & P51532-1 & isoform 1 & isoform B & & 1647 & NM_003072.3 & NP_003063.2 \\
\hline SMARCA4 & P51532-2 & isoform 2 & isoform $\mathrm{E}$ & 1259-1291: Missing. & 1614 & NM_001128847.1 & NP_001122319.1 \\
\hline SMARCA4 & P51532-3 & isoform 3 & isoform D & $\begin{array}{l}\text { 1259-1291: Missing; } \\
\text { 1388-1388: W } \rightarrow \text { WLKT; } \\
\text { 1475-1475: Missing. }\end{array}$ & 1616 & NM_001128846.1 & NP_001122318.1 \\
\hline SMARCA4 & P51532-4 & isoform 4 & isoform C & $\begin{array}{l}\text { 1259-1291: Missing; } \\
\text { 1388-1388: W } \rightarrow \text { WLKT }\end{array}$ & 1617 & NM_001128845.1 & NP_001122317.1 \\
\hline SMARCA4 & P51532-5 & isoform 5 & isoform $\mathrm{F}$ & $\begin{array}{l}\text { 1259-1291: Missing; } \\
\text { 1475-1475: Missing. }\end{array}$ & 1613 & NM 001128848.1 & NP 001122320.1 \\
\hline
\end{tabular}

b
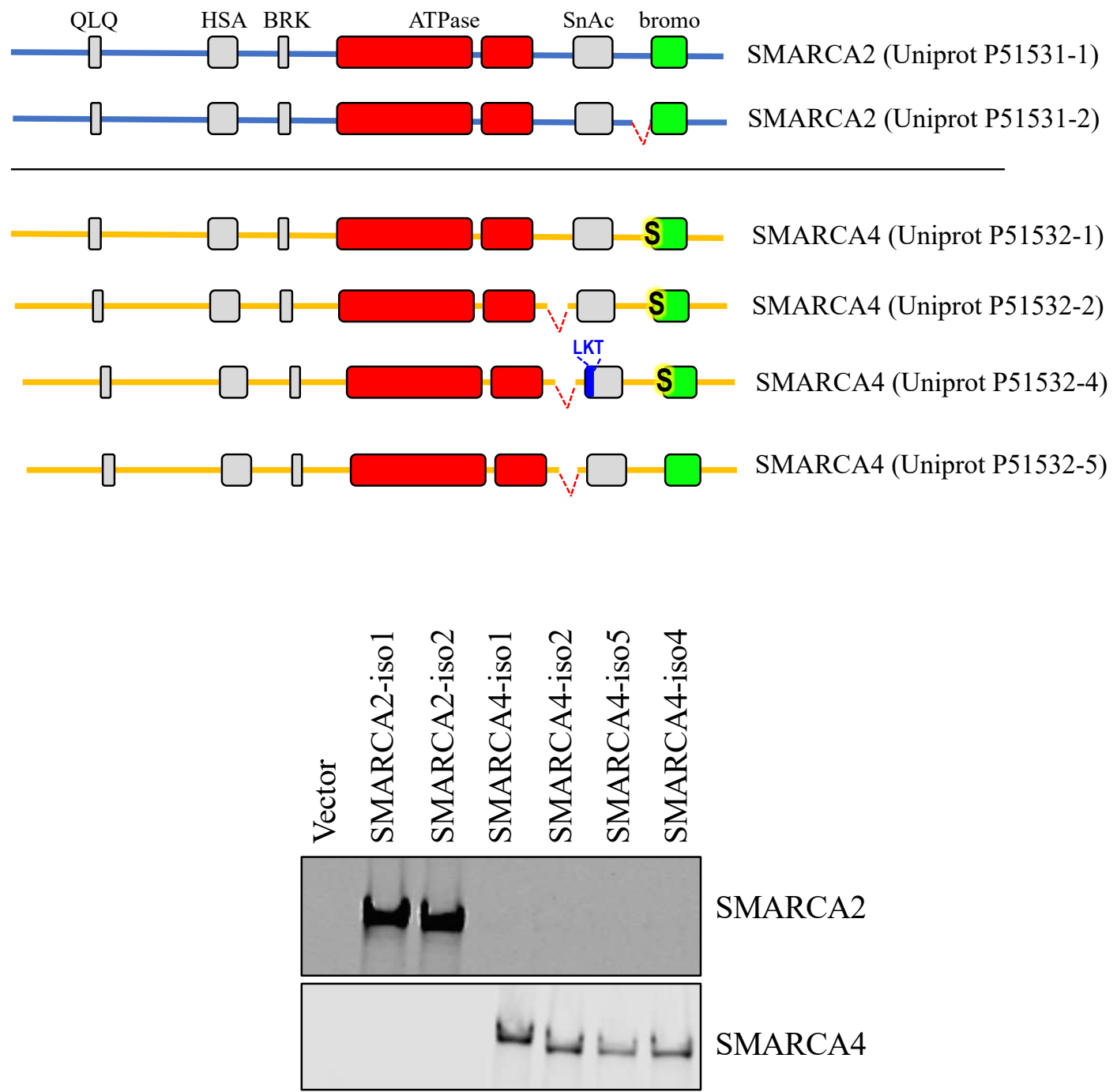

SMARCA2

SMARCA4 


\section{Extended Data Fig. 3.}
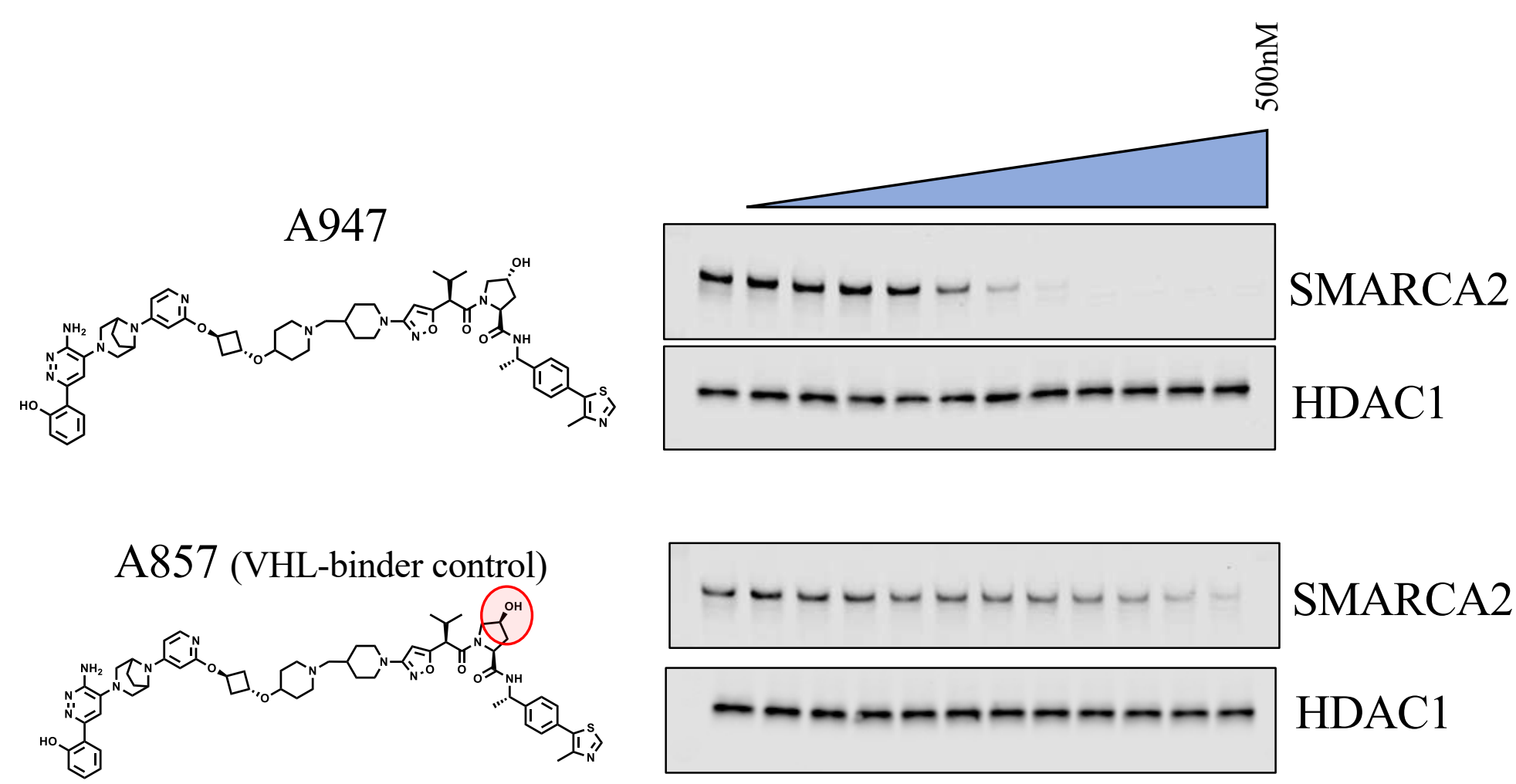

SMARCA2

A858 (SMARCA2-binder control)
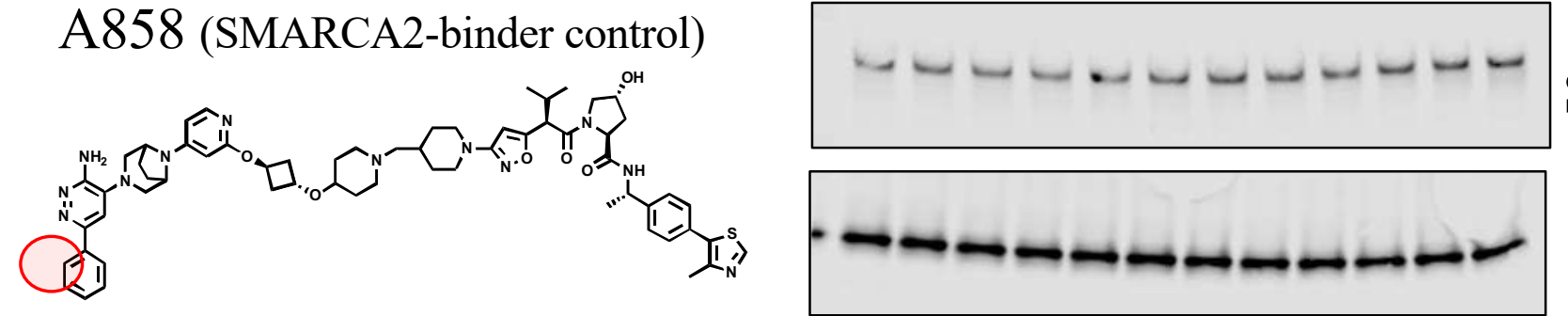

SMARCA2

HDAC1 
a.

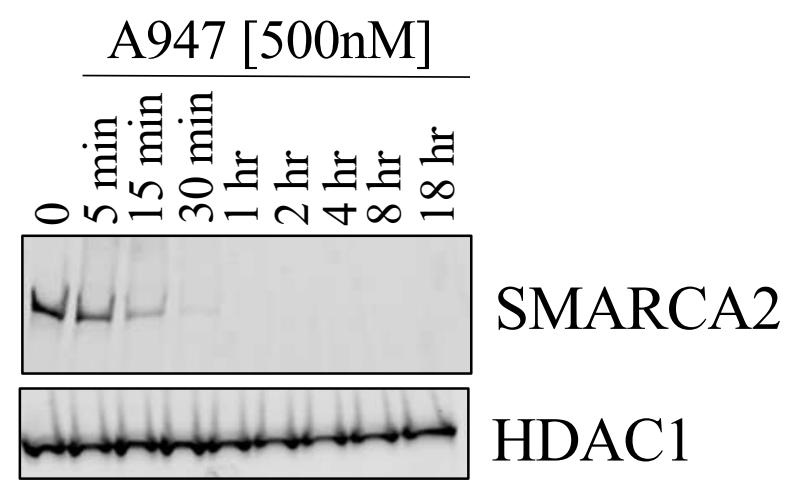

b.

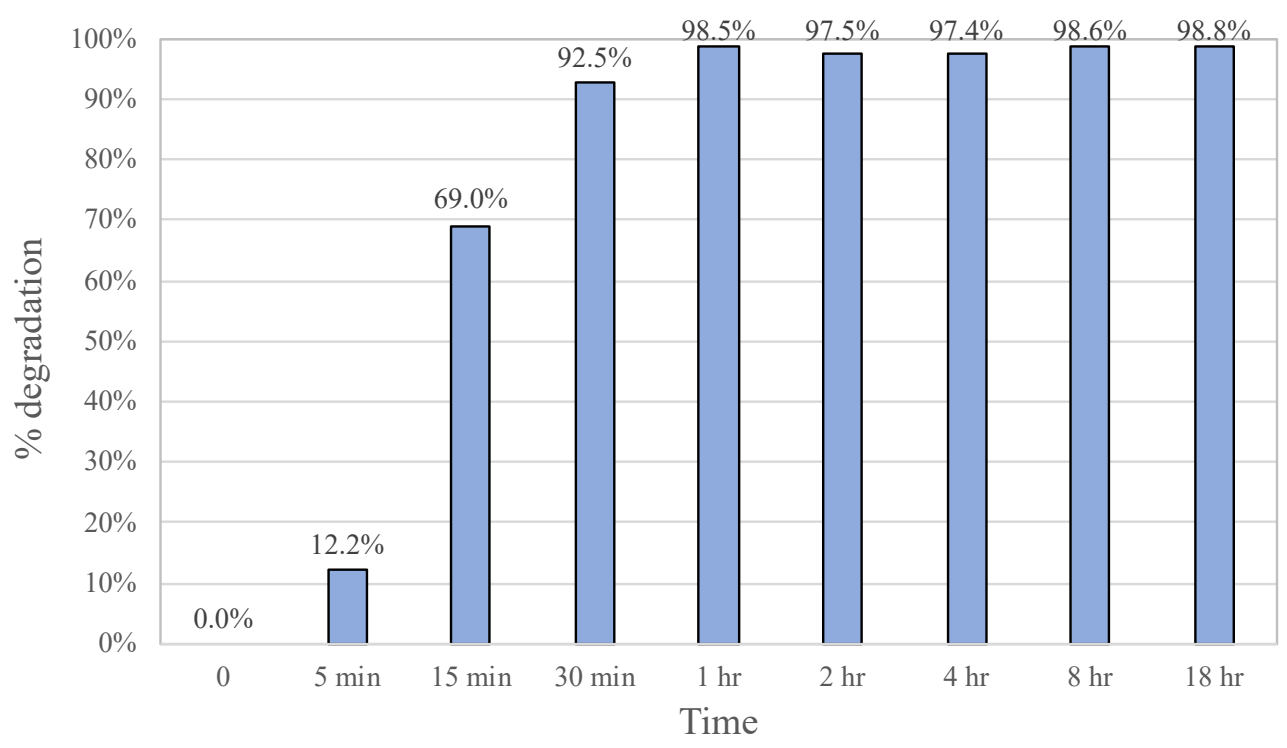

c.

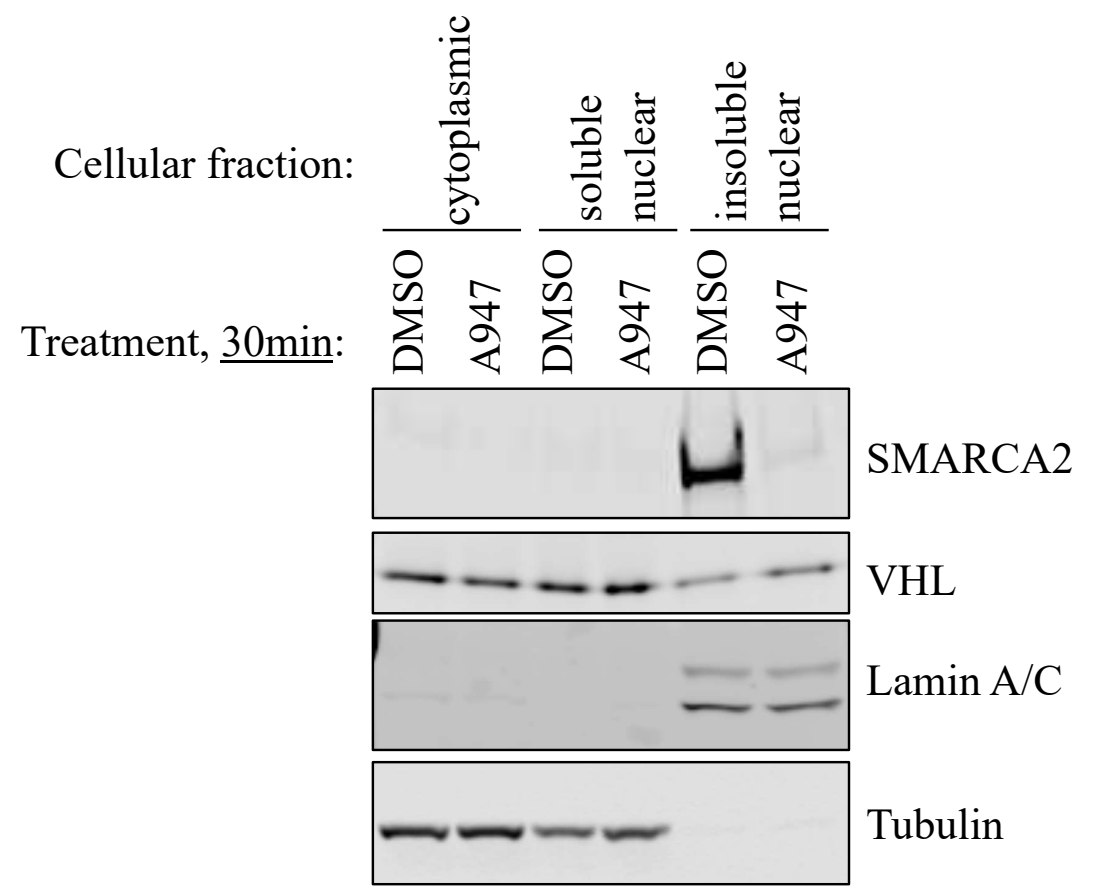




\section{Extended Data Fig. 5.}

$\mathbf{a}$
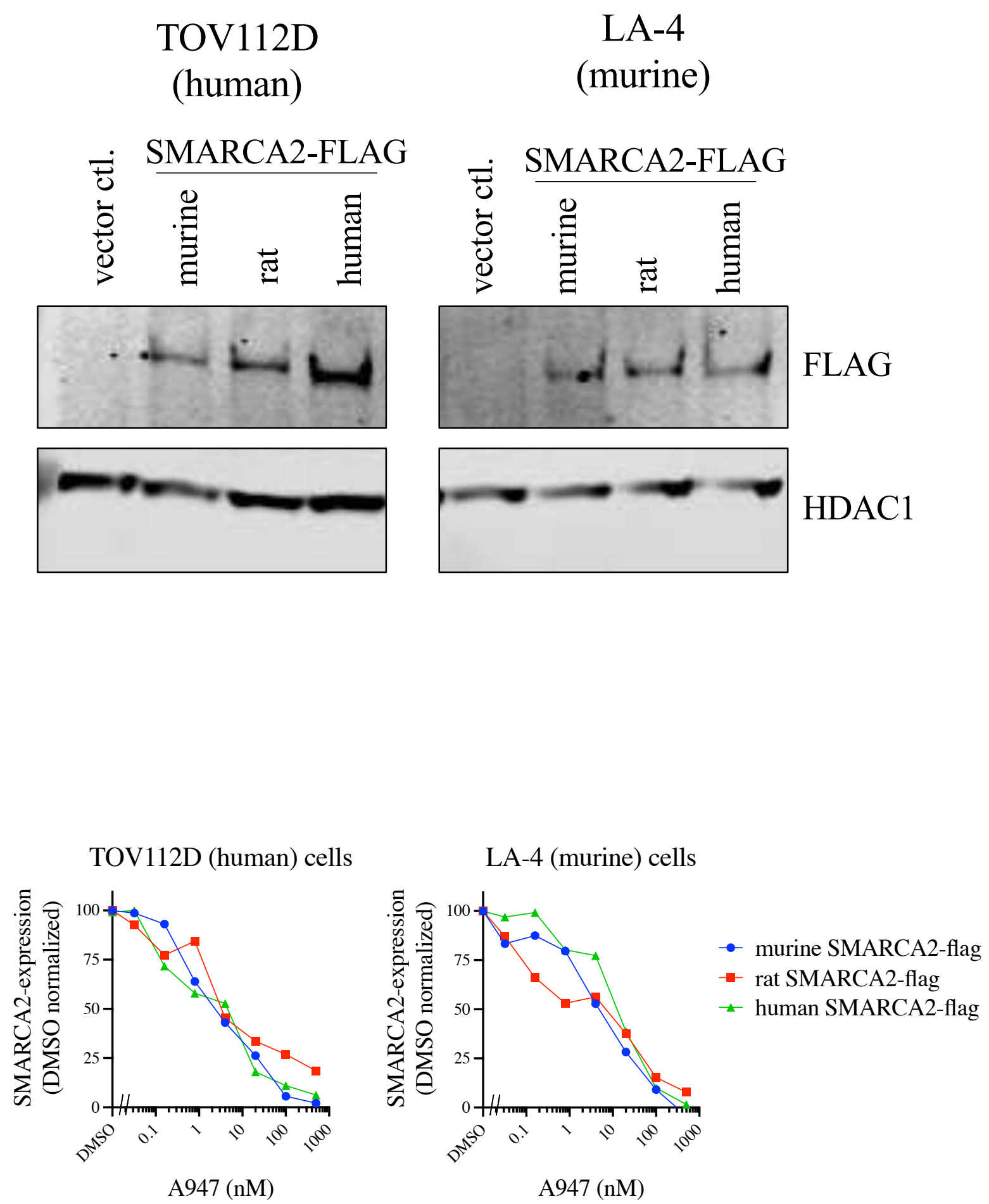


\section{Extended Data Fig. 6.}

a

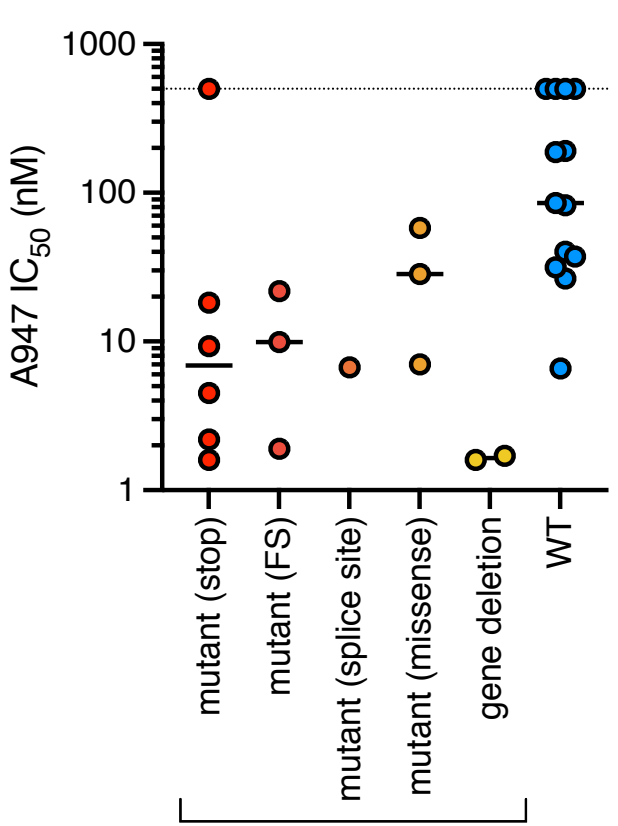

SMARCA4 variant type b

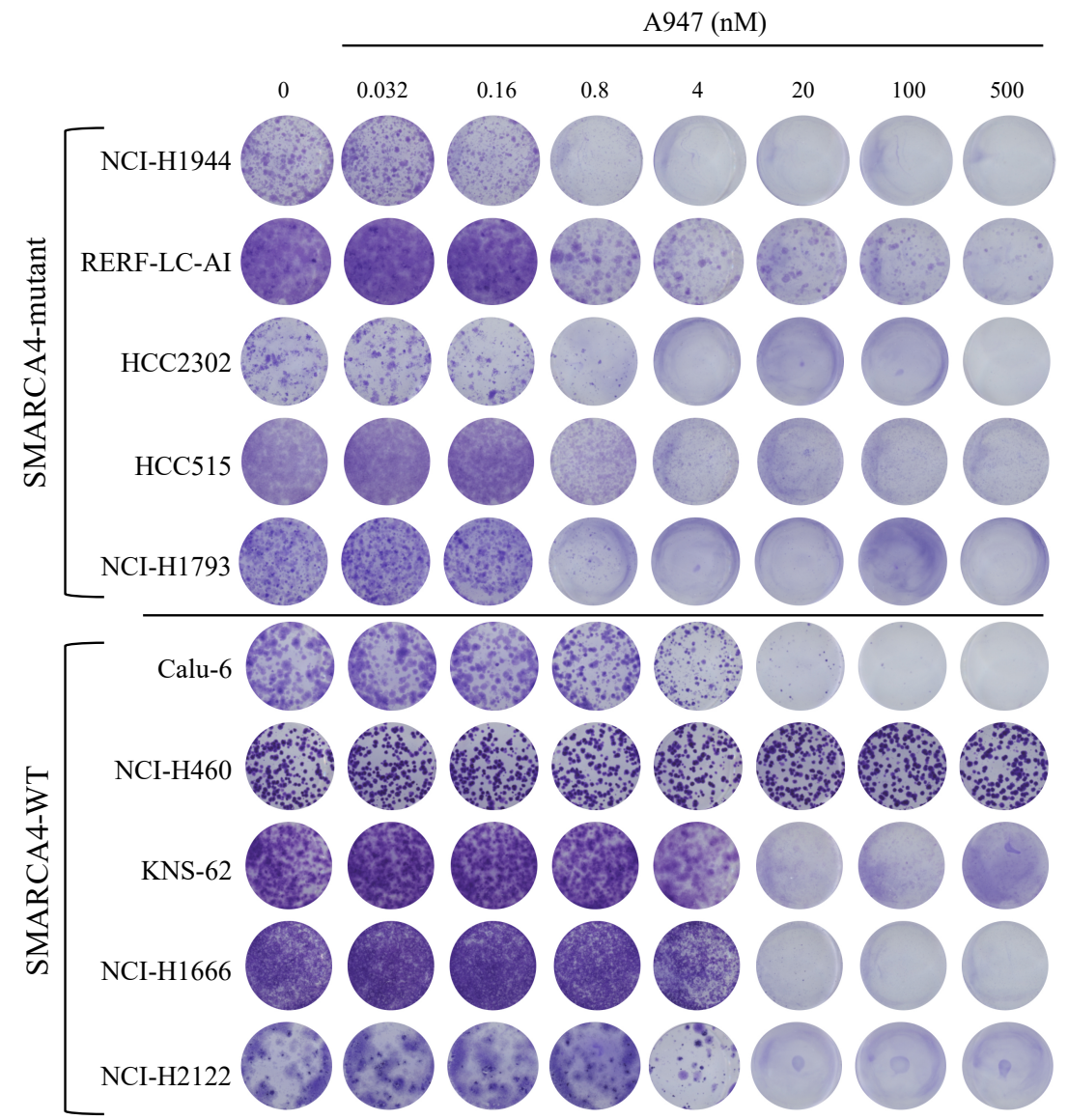

c

SMARCA4-wt

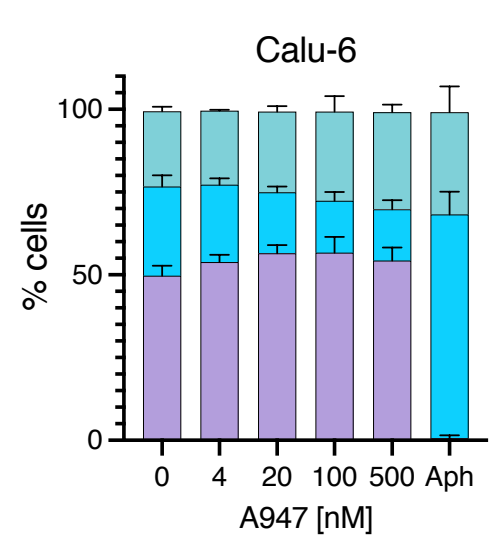

SMARCA2-negative

A427 (control)
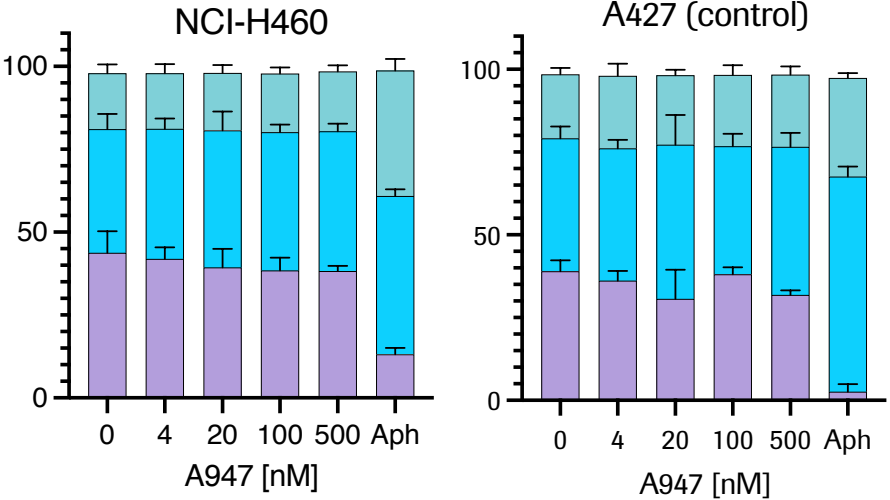

$\square \mathrm{S}$

$\square$ G1

$\square$ G2 


\section{Extended Data Fig. 7.}

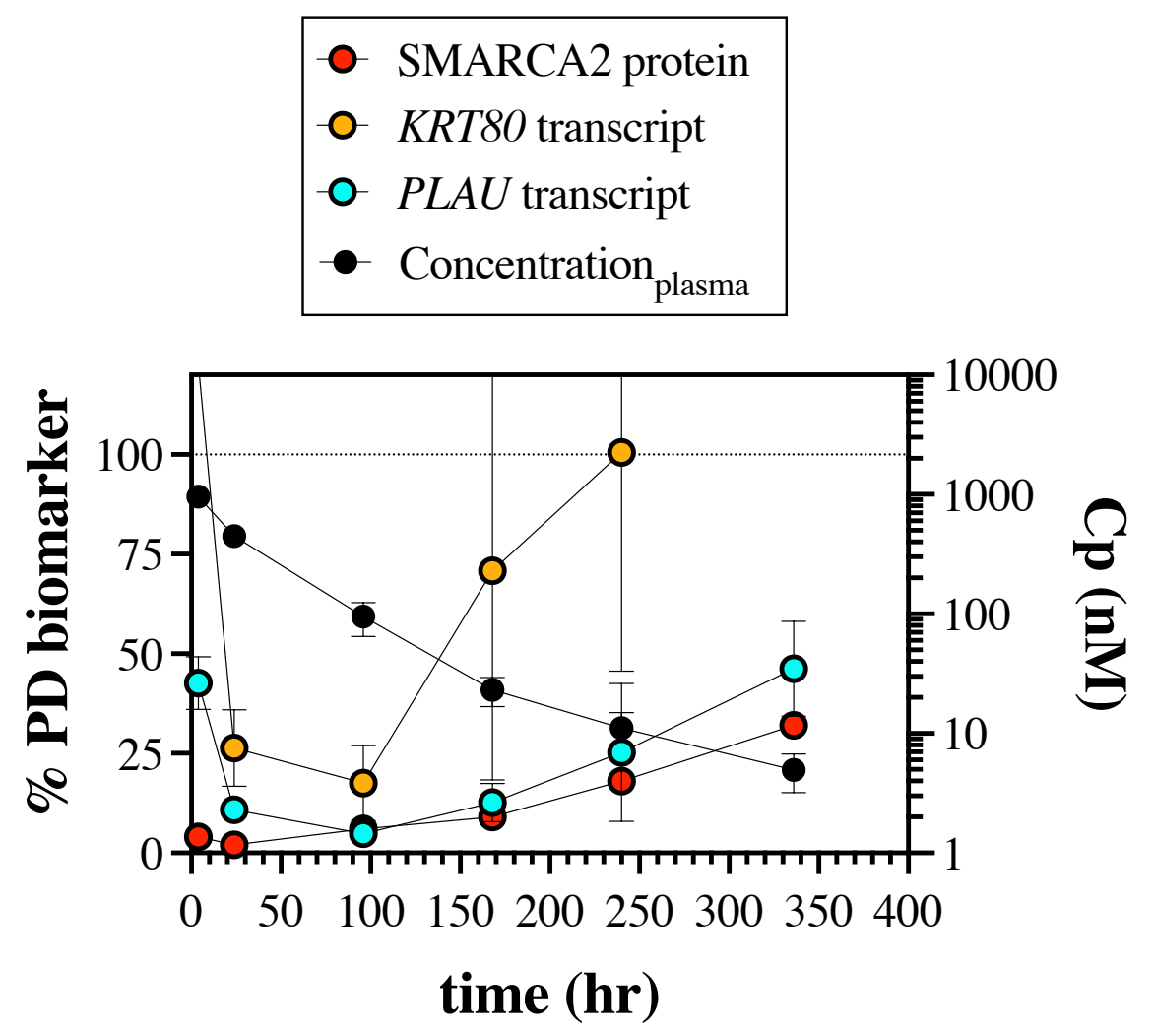




\section{Extended Data Fig. 8.}

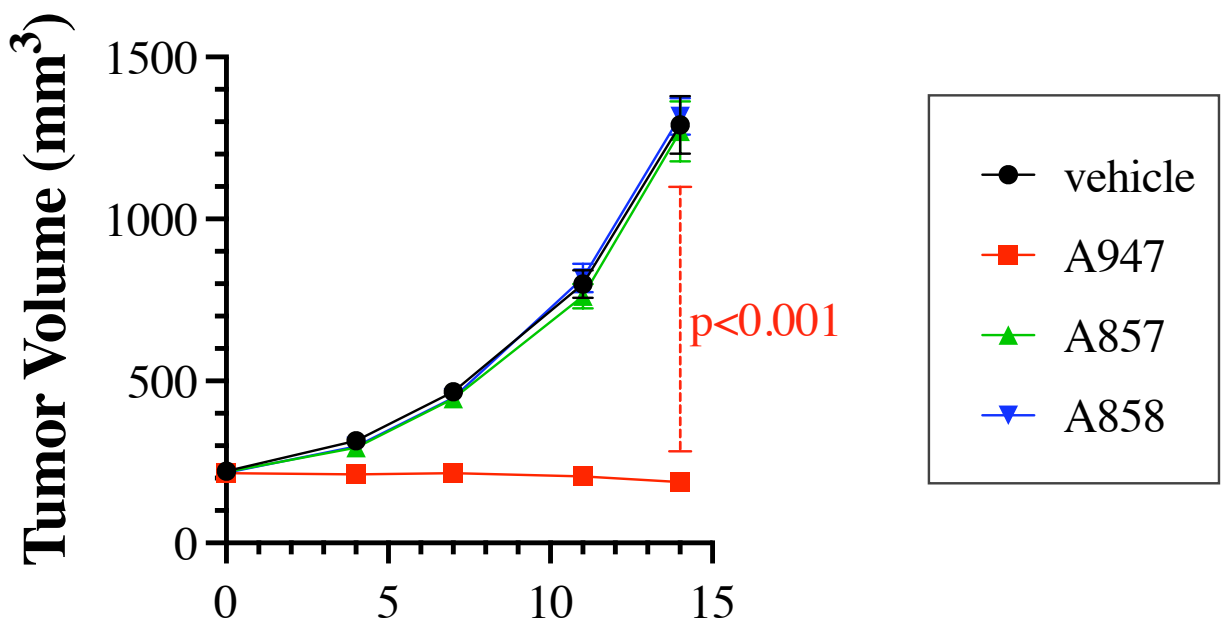

Days of tumor growth 


\section{Extended Data Fig. 9.}

$\mathbf{a}$

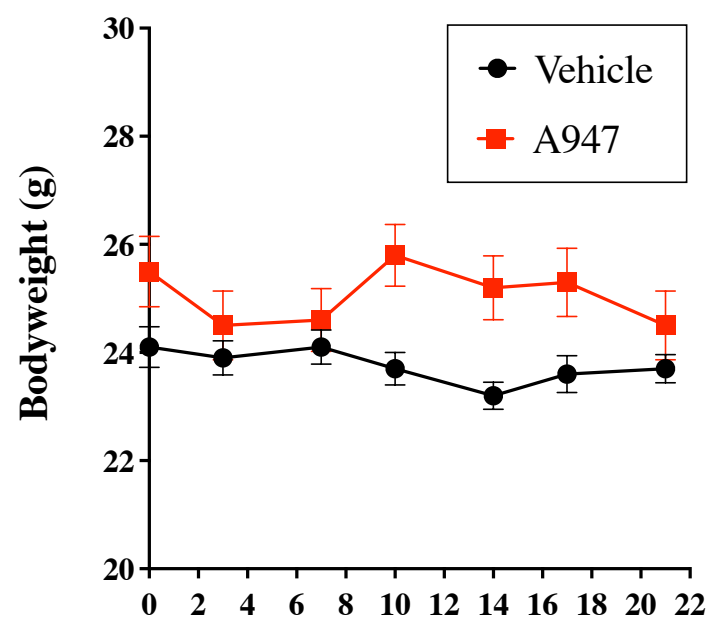

Days of tumor growth

b

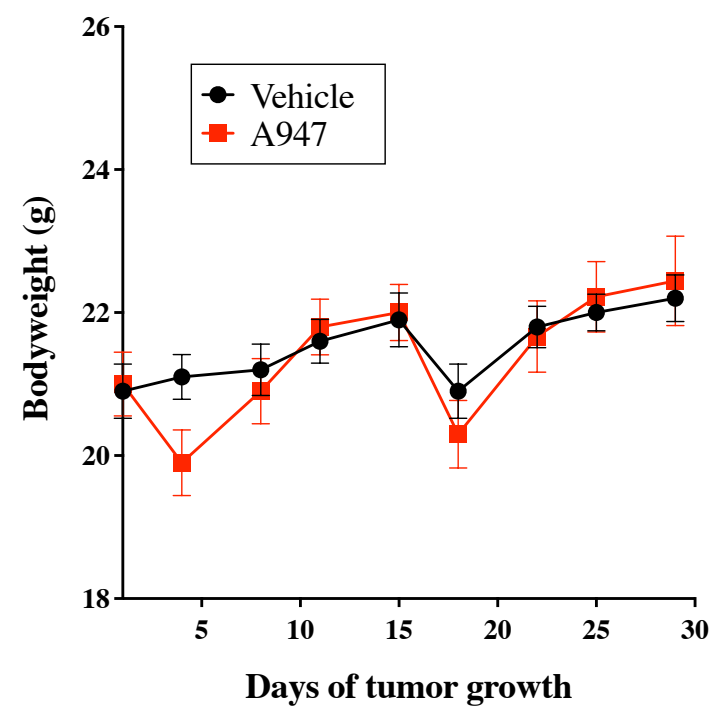

c

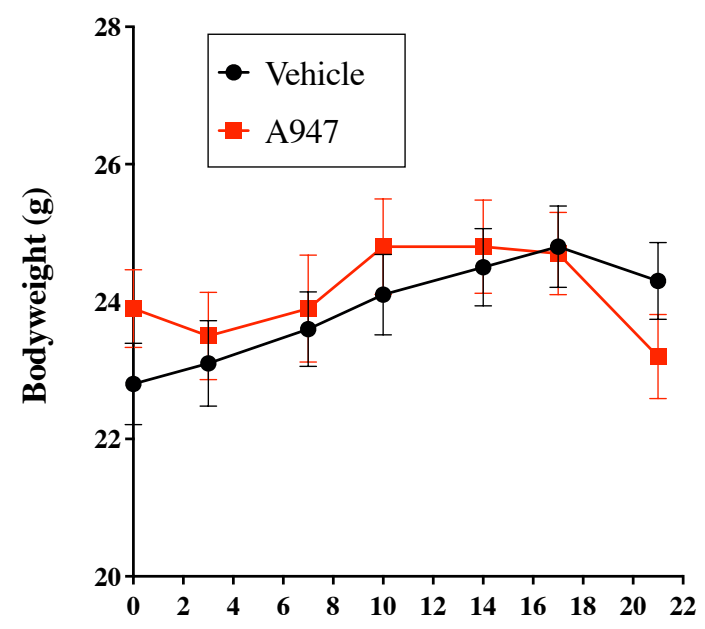

Days of tumor growth 


\section{Extended Data Fig. 10.}

a

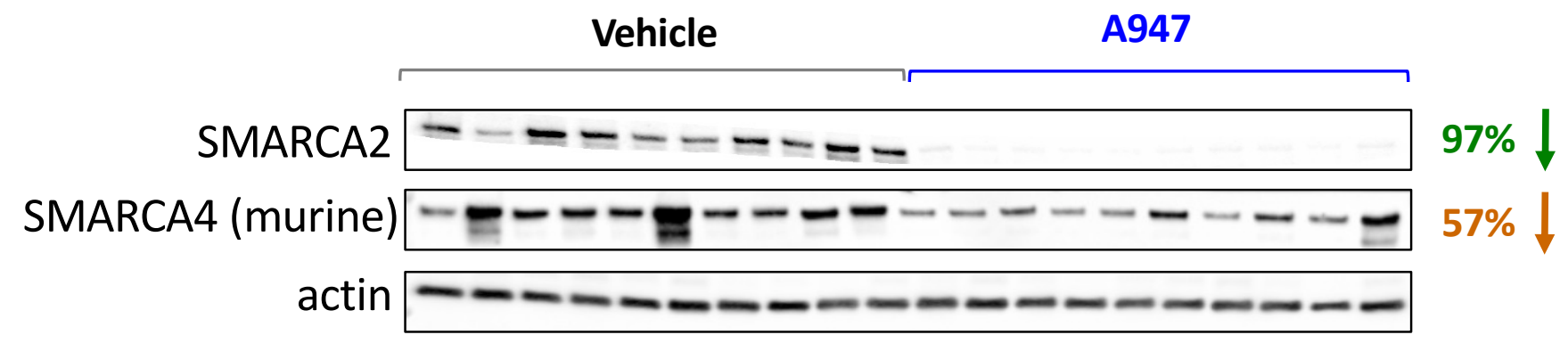

b

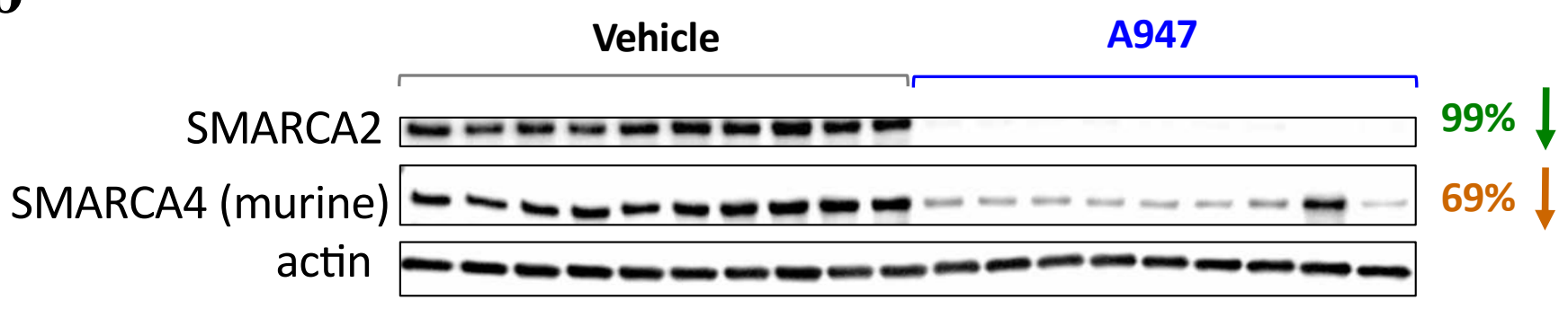




\section{Extended Data Fig. 11.}

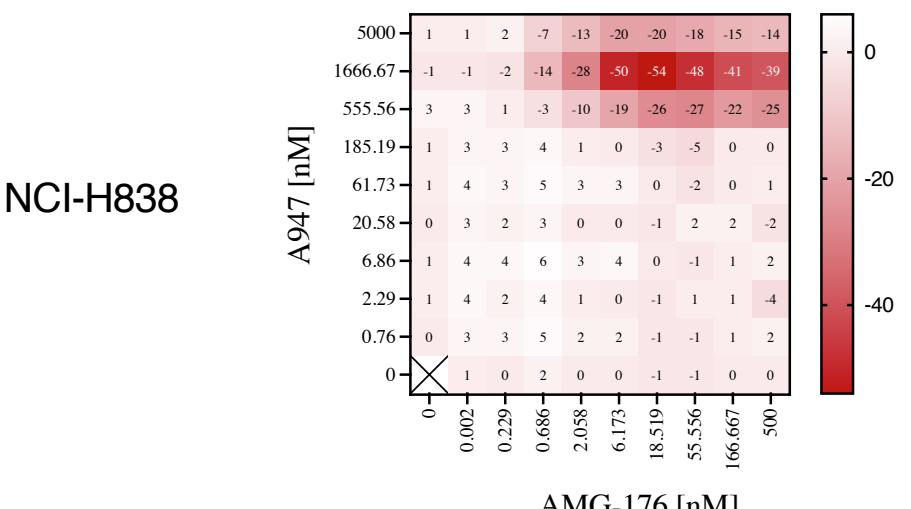

AMG-176 [nM]

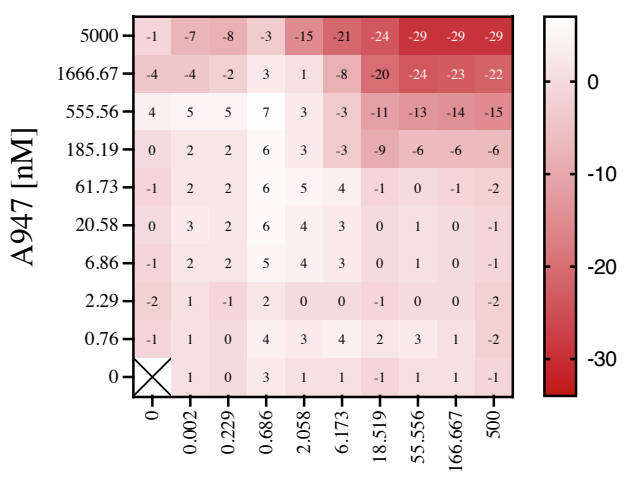

AMG-176 [nM]

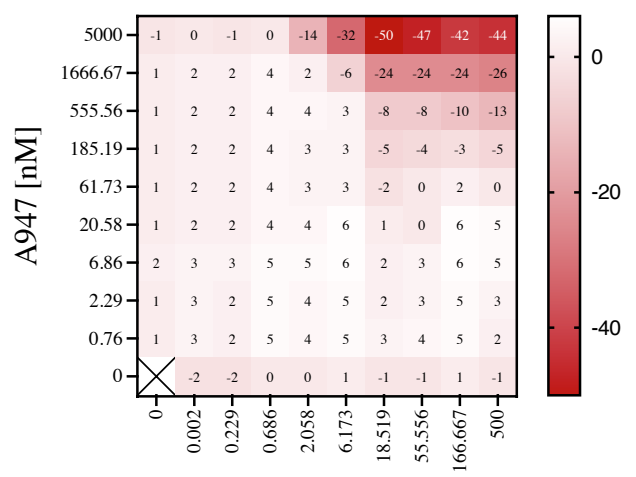

AMG-176 [nM]

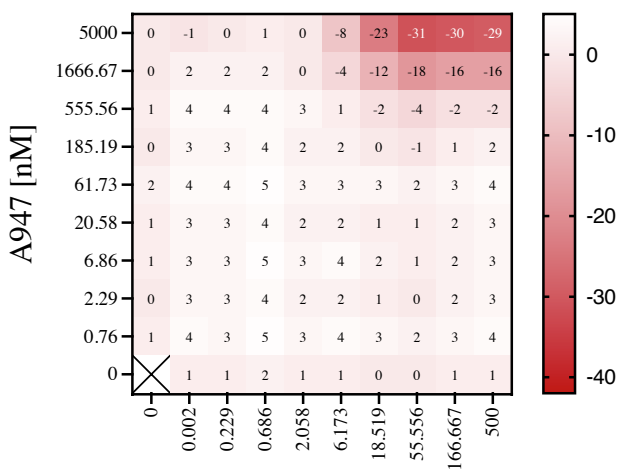

AMG-176 [nM]
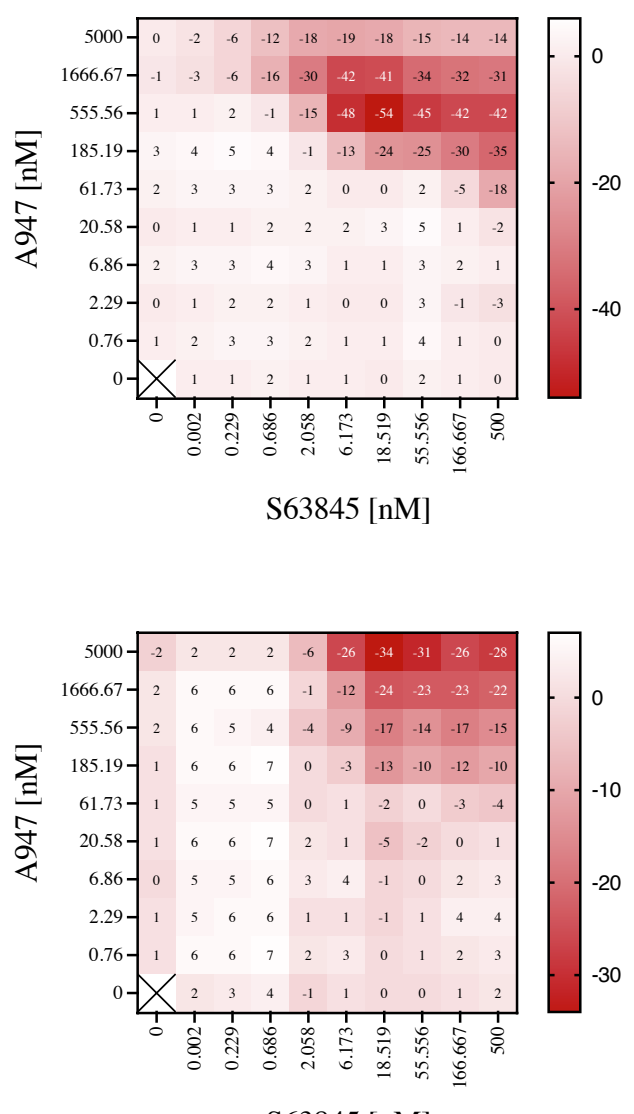

S63845 [nM]
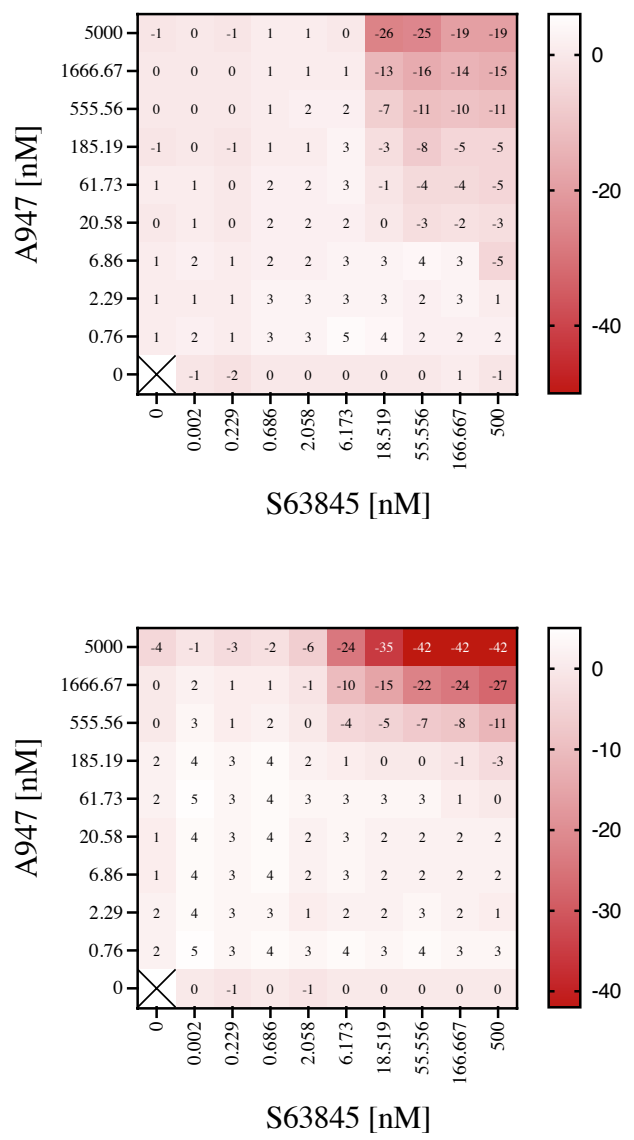